\title{
ESTUDO DE TENSŌES PELO MÉTODO DAS LINHAS DE FLUÊNCIA
}

DISSERTAÇĀO APRESENTADA PARA A OBTENÇĀO DO TÍTULO DE MESTRE EM ENGENHARIA MECÂNICA.

ORIENTADOR-

PROF. Dr. ALFREDO OTTO REHDER

\author{
. SĀO CARLOS \\ UNIVERSIDADE DE SĀO PAULO \\ CAMPUS DE SĀO CARLOS \\ ESCOLA DE ENGENHARIA DE SĀO CARLOS
}



ra a obtenção do titulo de mes tre em Engenharia Mecânica

$$
\text { Orientador- Prof. Dr. Otto }
$$

Alfredo Rehder

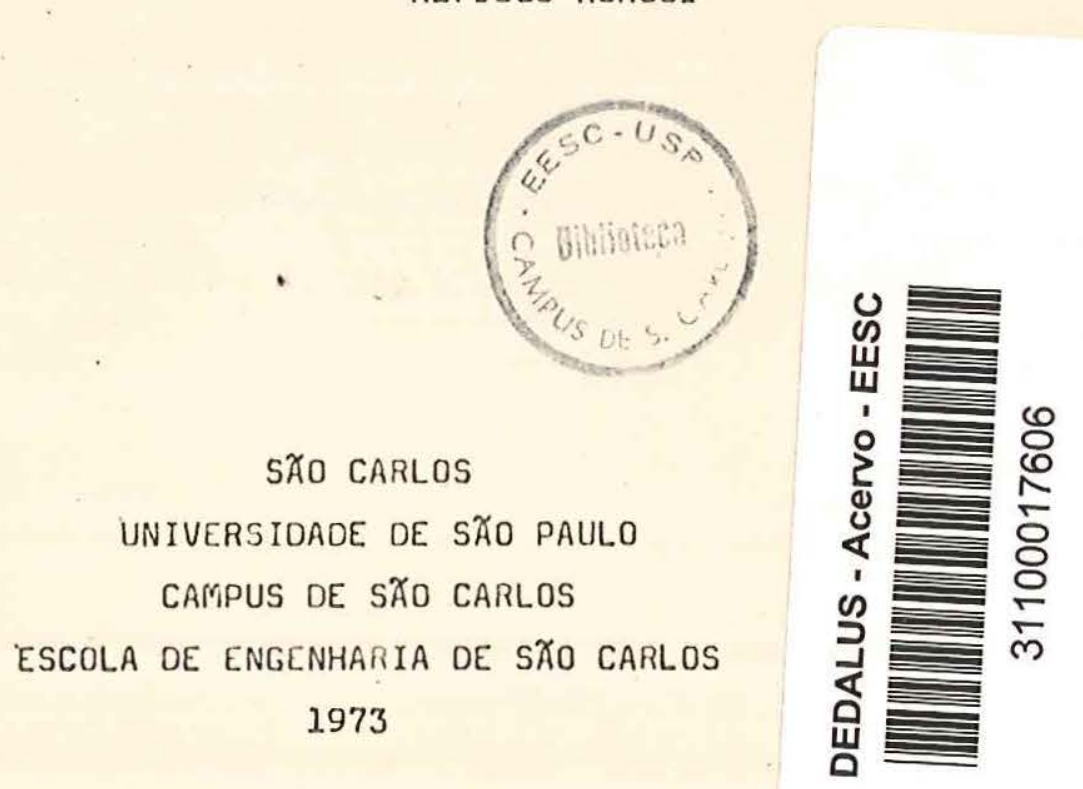


AGRADEC IMENTOS

Queremos nesta oportunidade agradecer a valiosa contribuição do Prof. Dr. Otto Alfredo fiehder pela orienta ção e incentivo a aos funcí onários do CPD pela amistosa dedicação. 
RESUTO

Trata-se de uma análise elasta plástica de tensões planas. nos métodios de conformação om forjanentos com matriz aberta ou rechada e trefil lasão. Empre ga-se o método das linhas de fluência, desenvolvido por Hencky e Prager. 0 estudio visa a determinação das tensões na região plástica e do campo de velocidades na região deformada, utilizando o método do Prager aplicado a um processo analítico. 
ABSTRACT.

An elastoplastic analysis of plane strain in axisymmetric plat and closed punch die die and wiredrawing was made uding the slip lines method, devellop ed by Hencky and Prager. Stu dies were made to determine the strains in the plastic zo ne and the velocities in de formed zone using the Prager's method applyed in an analitical process。 


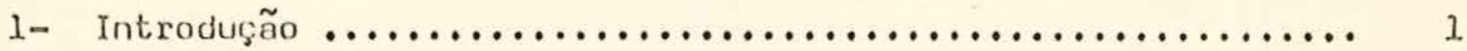

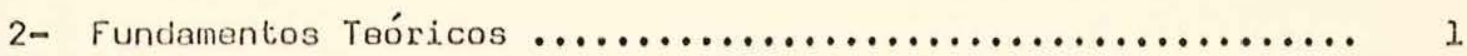

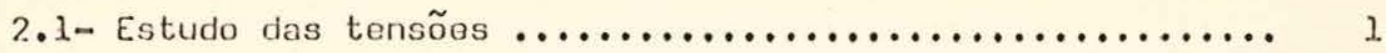

2.2- Estudo das tensões no ponto $\ldots \ldots \ldots \ldots \ldots \ldots \ldots \ldots \ldots \ldots$. 7

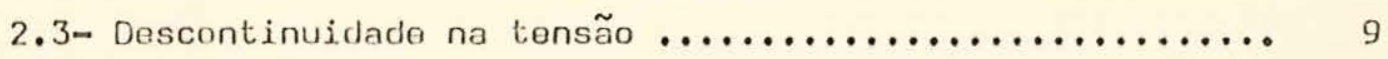

2.4- Estudo de tensões ao longo da linha de fluência ....... 1.1.

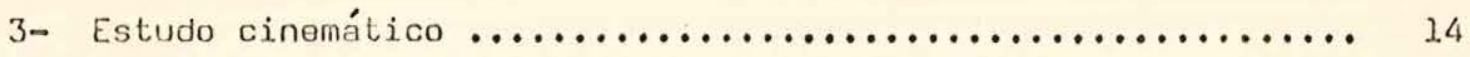

3.1 - Campo de velociclades $\ldots \ldots \ldots \ldots \ldots \ldots \ldots \ldots \ldots \ldots \ldots \ldots \ldots \ldots$

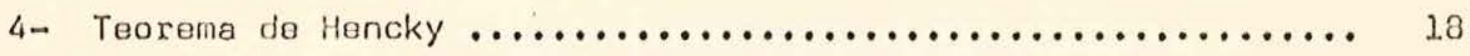

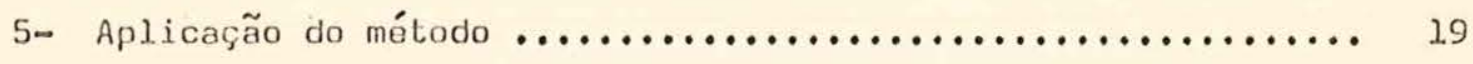

5.1 - Determinação dos pontos interseç̧ão das linhas ....... 19

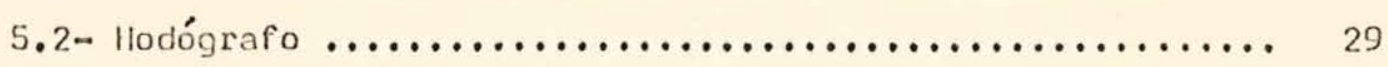

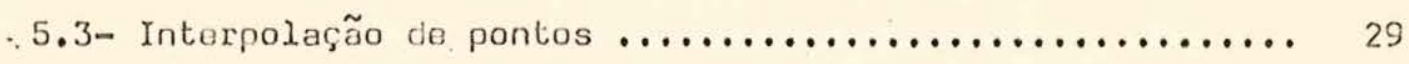

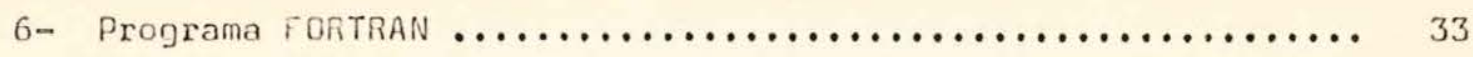

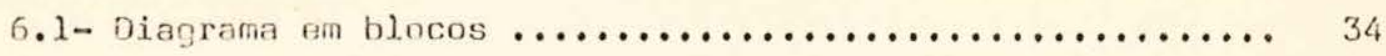

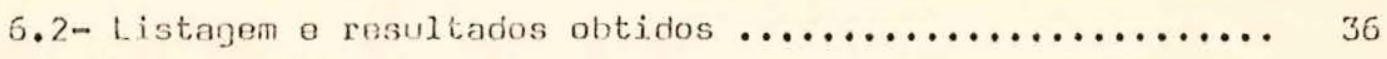

6.2 .1 - Pontos da malha de linhas de fluência ........ 44

6.2.2- Pontos interpolados ................... 48

6.2 .3 - Tensões, áreas e formas nos trechos ......... 55

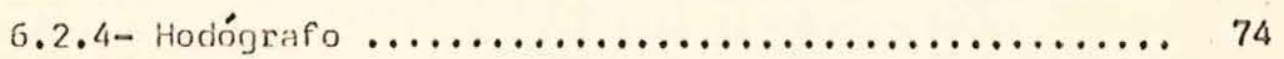

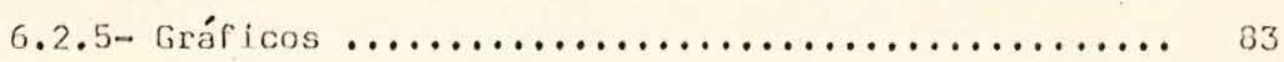

.6.3- rodo de operax o programa $\ldots \ldots \ldots \ldots \ldots \ldots \ldots \ldots \ldots \ldots \ldots$

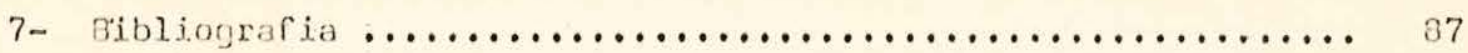


1- Introdução

Os problomas sobre deformação plástica recoberam uma atenção considerável após os trabalhos de Prandl em 1920. Prandl sugeriu um campo de linhas de cisalhamento como solução para forjamento com ma triz aberta, supondo que o material imediatamente abaixo da punção ficas se rígido e indeformável. Hill e outros propuseram uma solução mais exa ta, levando em conta o campo de velocidades de deformação. Hencky e Prager desenvolveram uma sistemática de estudo que permite determinar as linhas de fluência (ou de máxima tensão de cișalhamento) por processos gráficos.

Tais processos poden dar uma boa precisão mas são muito demorados pois baseiam-se numa sistemática de tentativas e erros.

A partir do teorema de Hencky, pode-se por meio de um estudo analítico, determinar os pontos de cruzamento das linhas onde é máxima a tensão de cisalhamento, assimilando os segmentos de linhas a segmentos de retas. Esta aproximação é valida para un número de pontos intersecção de curvas até 225 correspondendo a 15 pontos em cada linha.

Os cálculos são feitos por computacior digital, per mitindo a rápida obtenção de resultadios.

2- Fundamentos teóricos

2.1- Estudio das tensões

Os planos onde existem deformações são aqueles de máxima tensão de cisałhahiento. Estes planos formarn un conjunto de, curvas chamadas linhas de fluência ou linhas de cisalhamento.

0 círculo de moHr correspondente a un ponto de um destes planos encontra-se representado na figura (1), onde $k$ é constante e as tensões estão determinadas pelo valor de $\tau_{m} \cdot 0$ elemento material em que estamos interessarlos não é rormado por coordenadas de sistemas cartezianos fixos, mas sim por um sistema de coordenadas curvilíneas , como mostrado na figura (2). 


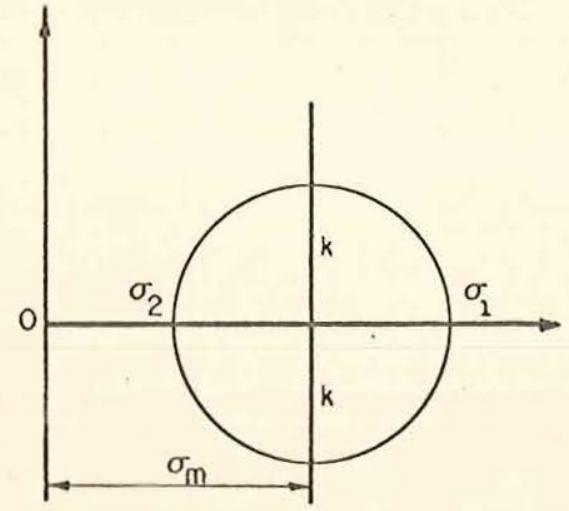

FIG.- 1

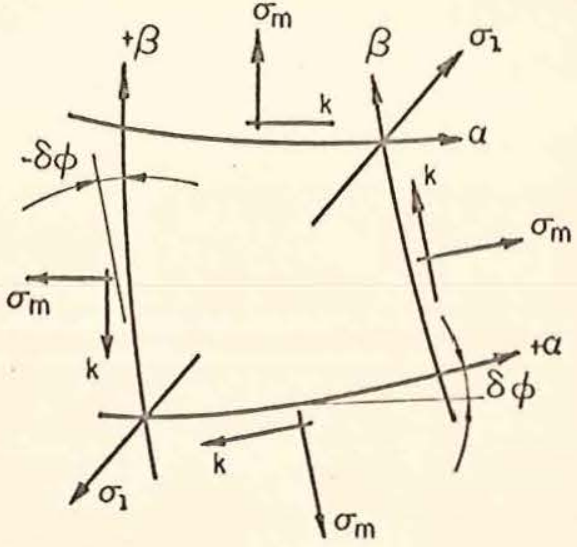

FIG -2

Os dois conjuntos de curvas, perpendiculares entre si, são chamados de famílias de curvas $\alpha$ e $\beta$. A convenção de sinal. adotada é a seguintes as direções de duas curvas $\alpha$ ç formam ângulós positivos entre si no sentido antihorário e todos os demais ângulos se rão considerados positivos no sentido antihorário quando tornados entre ad direções positivas de dois ejxos quaisquer.

\section{Supõe-se agora o elemento $A B C D$, com os contornos} determinados pelas linhas $A D$ e BC pertencentes à uma família de curvas e „pelas linhas'DC e $A B$ pertencentes à uma família de curvas $\beta$. Supõ日-se ainda que as famílias $\alpha c \beta$ tenham as suas curvas ortogonais entre sj. As curvas $A D, B C, D C$ a $A B$ foram determinadas a partir do ponto 0 (determinado pela intersecção de curvas das famílias $\alpha$ e $\beta$ ) com os incrementos, $\pm \Delta \dot{\alpha} / 2$ e $\pm \Delta \beta / 2$, como mostrado na figura (3).

Considera-se o eixo z normal a xy e o ângulo en tre yz é positivo quando se tomam ângulos no sentido antihorário. 0 ele mento ABCD é considerado suficientemente pequeno para que suas faces for mem planos paralelos a $x y$ e além disso a altura $z$ do elemento é conside rada constante e unitária. 


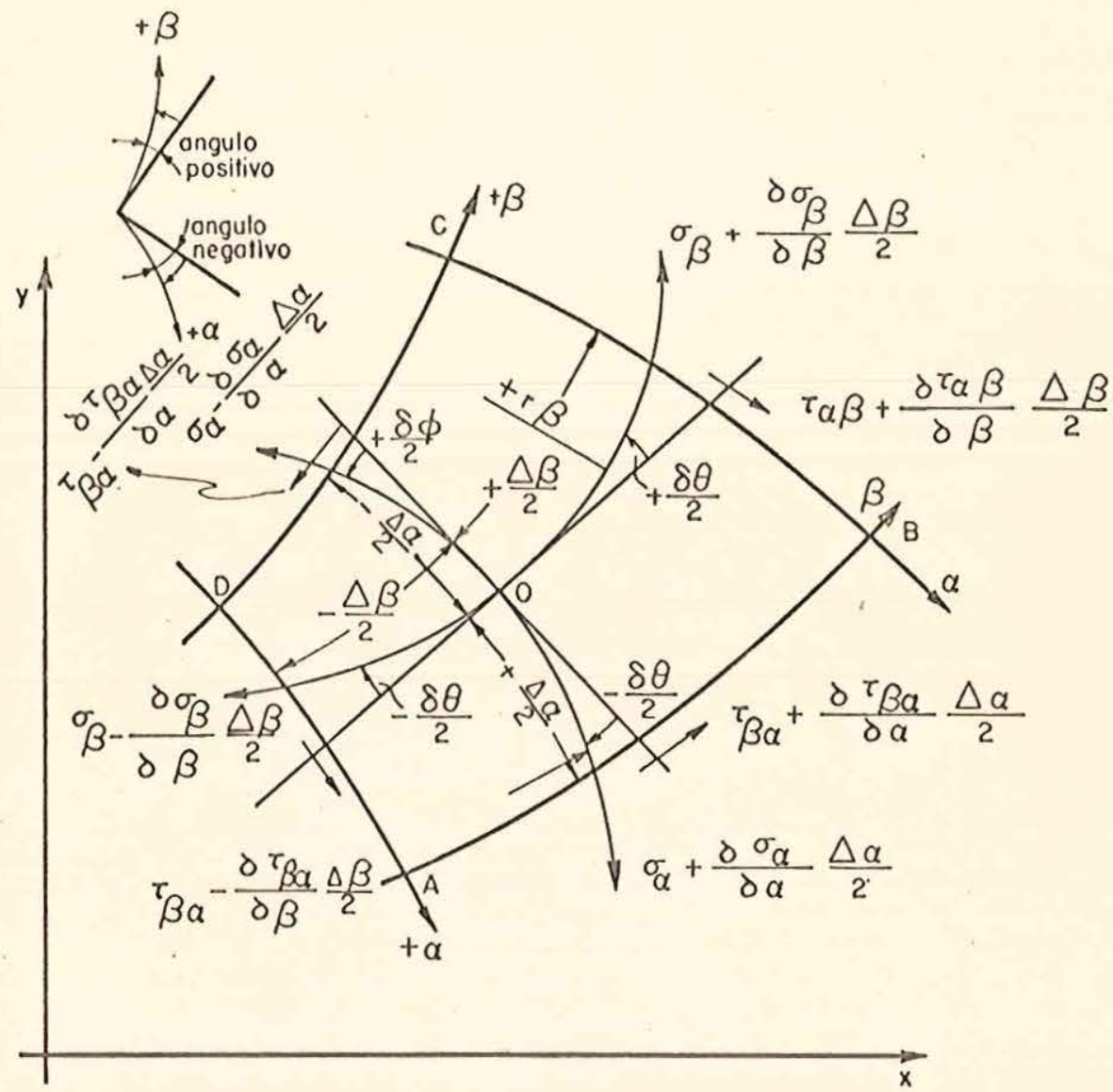

FIG -3

As curvas estão orientadas segundo as direções po sitivas indicadas na figura (3), в os ârgulos são positivos no sentido antihorário.

Os ângulos $\delta \theta$ e $S \phi$ são tomados das tangentes pa ra a curva a os raios $r_{\beta}$ e $r_{\alpha}$ serão positivos ou negativos conforme des crevan ângulos positivos ou negativos a partir do ponto U. para a curva que os definem. Na direção positiva das curvas $\propto$ a fôrça normal $F \propto+$ tem componente:

$$
F_{\alpha+}=\left(\sigma_{\alpha}+\frac{\partial \sigma_{\alpha}}{\partial \alpha} \cdot \frac{\Delta \alpha}{2}\right) \cdot 1 \cdot A B \cdot \cos \left(\frac{-\delta \phi}{2}\right)
$$


Na direção negativa será:

$$
F_{\alpha-}=\left(\sigma_{\alpha} \div \frac{\partial \sigma_{\alpha}}{\partial \alpha} \frac{\Delta \alpha}{2}\right) \cdot L \cdot C D \cdot \cos \left(\frac{1 \delta \phi}{2}\right)
$$

0 ângulo entre as curvas DA e CB (considerando-se que os arcos compreendern ângulos muito pequenos) será $S \theta$. E também:

$$
\begin{aligned}
r_{\beta} S \theta & =\Delta \beta \\
\text { o raio de curvatura de } A B \text { será: } & \\
r_{A B} & =r_{\beta}+\frac{\Delta \alpha}{2} \\
A B & =r_{A B} \delta \theta=\left(r_{\beta}+\frac{\Delta \alpha}{2}\right) \delta \theta
\end{aligned}
$$

ou

$$
A B=\Delta \beta+\frac{\Delta \alpha \Delta \beta}{2 \beta}
$$

da mesma maneira,

$$
\delta \phi=\frac{\Delta \alpha}{r_{\alpha}}
$$

e como

$$
\cos \left(\frac{-\delta \phi}{2}\right)=\cos \left(+\frac{\delta \phi}{2}\right) \simeq 1
$$

B

$$
C D=\Delta \beta-\frac{\Delta \alpha \Delta \beta}{2 \Gamma \beta}
$$

Substituindo os valores de $A B$ a CD nas equações

(1) e (2), teremos a componente de $F_{\alpha}$ na direção $\propto$ :

$$
\begin{aligned}
c \sigma_{\alpha}= & \left(\sigma_{\alpha}+\frac{\partial \sigma_{\alpha}}{\partial \alpha} \cdot \frac{\Delta \alpha}{2}\right) \cdot\left(\Delta \beta+\frac{\Delta \alpha \Delta \beta}{2 r_{\beta}}\right)- \\
& -\left(\sigma_{\alpha}-\frac{\partial \sigma_{\alpha}}{\partial \alpha} \frac{\Delta \alpha}{2}\right) \cdot\left(\Delta \beta-\frac{\Delta \alpha \Delta \beta}{2 r_{\beta}}\right)
\end{aligned}
$$

As forças nas direções das linhas $\beta$ terão componentes nas direções das linhas $\alpha$ pois as $F_{\beta}$ в seus incrementos estão inclinados de $(\pi / 2+\delta \theta / 2)$ em relação às linhas $\propto$. Portanto a resultanto será:

$$
\begin{aligned}
C \sigma_{\beta}=- & {\left[\left(\sigma_{\beta}+\frac{\partial \sigma_{\beta}}{\partial \beta} \frac{\Delta \beta}{2}\right) \cdot B C \cdot 1 \cdot \operatorname{sen}\left(\frac{S \theta}{2}\right)+\right.} \\
+ & \left.\left(\sigma_{\beta}-\frac{\partial \sigma_{\beta}}{\partial \beta} \frac{\Delta \beta}{2}\right) A D \cdot 1 \cdot \sin \left(\frac{\delta \theta}{2}\right)\right]
\end{aligned}
$$


e com

$$
\begin{aligned}
& B C=\left(-r_{\alpha}+\frac{\Delta \beta}{2}\right) \delta \phi=\Delta \alpha-\frac{\Delta \alpha \Delta \beta}{2 r_{\alpha}} \\
& A D=\left(-r_{\alpha}-\frac{\Delta \beta}{2}\right) S \phi=\Delta \alpha+\frac{\Delta \alpha \Delta \beta}{2 r_{\alpha}} \\
& \sin \frac{\delta \theta}{2} \simeq \frac{S \theta}{2} \simeq \frac{\Delta \beta}{2 r_{\beta}}
\end{aligned}
$$

A resultante da força devido as tensões da direção $\beta$ como componente na direção $\alpha$ será:

$$
\begin{aligned}
c \sigma_{\beta}= & -\left[\left(\sigma_{\beta}+\frac{\partial \sigma_{\beta}}{\partial \beta} \frac{\Delta \beta}{2}\right)\left(\Delta \alpha-\frac{\Delta \alpha \Delta \beta}{2 r_{\alpha}}\right) \frac{\Delta \beta}{2 r_{\beta}}+\right. \\
& +\left(\sigma_{\beta}-\frac{\partial \sigma_{\beta}}{\partial \beta} \frac{\Delta \beta}{2}\right)\left(\Delta \alpha+\frac{\Delta \alpha \Delta \beta}{2 r_{\alpha}}\right) \frac{\Delta \beta}{2 r_{\beta}}
\end{aligned}
$$

A força devido a tensão de cizalhamento na face $A B$ tem como componente na direção $\alpha$ :

$$
C_{\beta \alpha \alpha}=\left(G_{\beta \alpha}+\frac{\partial \sigma_{\beta \alpha} \Delta \alpha}{\partial \alpha}\right)\left(\Delta \beta+\frac{\Delta \alpha \Delta \beta}{2 r_{\beta}}\right) \operatorname{Sen}\left(\frac{-\delta \phi}{2}\right)
$$

Na face CD será:

$$
c^{\prime} \zeta_{\beta \alpha}=\left(\zeta_{\beta \alpha}-\frac{\partial \sigma_{\beta \alpha} \alpha}{\partial \alpha} \frac{\Delta \alpha}{2}\right)\left(\Delta \beta-\frac{\Delta \alpha \Delta \beta}{2 r_{\beta}}\right) \operatorname{sen}\left(\frac{+\delta \phi}{2}\right)
$$

Para ambas as faces

$$
\operatorname{Sen}\left(\frac{ \pm S \phi}{2}\right) \simeq \delta \phi \simeq-\frac{\Delta \alpha}{r \alpha}
$$

Na face DA

$$
C \zeta_{\alpha \beta}=-\left(\zeta_{\alpha \beta}-\frac{\partial \zeta_{\alpha \beta}}{\partial \beta} \frac{\Delta \beta}{2}\right)\left(\Delta \alpha+\frac{\Delta \alpha \alpha_{\beta}}{2 r_{\alpha}}\right)
$$

$\mathrm{Na}$ face $\mathrm{BC}$

$$
C^{\prime} \sigma_{\alpha \beta}=\left(\zeta_{\alpha \beta}+\frac{\partial_{\alpha \beta}}{\partial \beta} \frac{\Delta \beta}{2}\right)\left(\Delta \alpha-\frac{\Delta \alpha \Delta \beta}{2 r_{\alpha}}\right)
$$

termos iguais a de ordem superior, teremos, na ausência de for ças externas: 
$-6$

$$
\begin{aligned}
& 2 \frac{\partial \sigma_{\alpha}}{\partial \alpha} \frac{\Delta \alpha \Delta \beta}{2}+2 \sigma_{\alpha} \frac{\Delta \alpha \Delta \beta}{2 \Gamma_{\beta}}-2 \sigma \beta \frac{\Delta \alpha \Delta \beta}{2 \sigma_{\beta}} \cdots \\
& -2 \frac{\partial \tau_{\alpha \beta}}{\partial \beta} \frac{\Delta \alpha \Delta \beta}{2}-\left(2 \zeta_{\beta \alpha} \frac{\Delta \alpha \Delta \beta}{2 r_{\alpha}}+2 \sigma_{\alpha \beta} \frac{\Delta \alpha \Delta \beta}{2 r_{\alpha}}\right)=0
\end{aligned}
$$

ou

$$
\frac{\partial \sigma_{\alpha}}{\partial \alpha}+\frac{\partial \sigma_{\alpha} \beta}{\partial \beta}+\frac{\sigma_{\alpha}-\sigma_{\beta}}{r_{\beta}}-2 \frac{\sigma_{\alpha \beta}}{\sigma_{\alpha}}=0
$$

para as linhas $\alpha$.

Da mesma maneira, para as linhas $\beta$ teremos:

$$
\frac{\partial \sigma_{\beta \beta}}{\partial \beta}+\frac{\partial \sigma_{\beta \alpha}}{\partial \alpha}+\frac{\sigma_{\beta}-\sigma_{\alpha}}{r_{\alpha}}+2 \frac{\sigma_{\alpha \alpha}}{r_{\beta}}=0
$$

supondo $\sigma_{\beta}=\sigma_{\alpha}=\sigma_{m}$ a $Z_{\alpha \beta}=\zeta_{\beta \alpha}=k$ teremos, pelas equações (22) e (23) respectivamente válidas para as linhas $\alpha$ e $\beta$ :

$$
\begin{aligned}
& \frac{\partial \sigma m}{\partial \alpha}+\frac{\partial k}{\partial \beta}-2 k \frac{\partial \phi}{\partial \alpha}=0 \text { (linines } \alpha \text { ) } \\
& \frac{\partial \sigma_{m}}{\partial \beta}+\frac{\partial k}{\partial \alpha}+2 k \frac{\partial \phi}{\partial \beta}=0(\operatorname{lin} \hat{\alpha} \theta \dot{\beta})
\end{aligned}
$$

integrando as equações (24) e (25):

$$
\begin{aligned}
& \sigma m-2 k \phi+\int \frac{\partial k}{\partial \beta} d \alpha=C \alpha(\text { linher } \alpha) \\
& \sigma m+2 k \phi+\int \frac{\partial k}{\partial \alpha} d \beta=c \beta(\text { in hor } \beta)
\end{aligned}
$$

para um material perfeitamente plástico:

$$
\frac{\partial k}{\partial \beta}=\frac{\partial k}{\partial \alpha}=0
$$

as equações (26) e (27) ficam:

$$
\begin{aligned}
& \sigma_{m}-2 k \phi=c_{\alpha}(\operatorname{linh} d \alpha) \\
& \sigma_{m}+2 k \phi=C_{\beta} \quad(\operatorname{linhe} \beta)
\end{aligned}
$$


As (29) в (30) são as equações de Hencky.

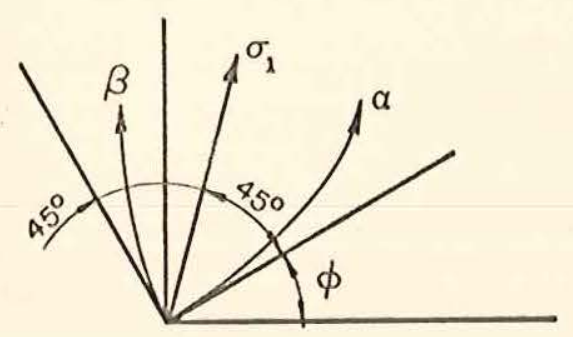

FIG. -5

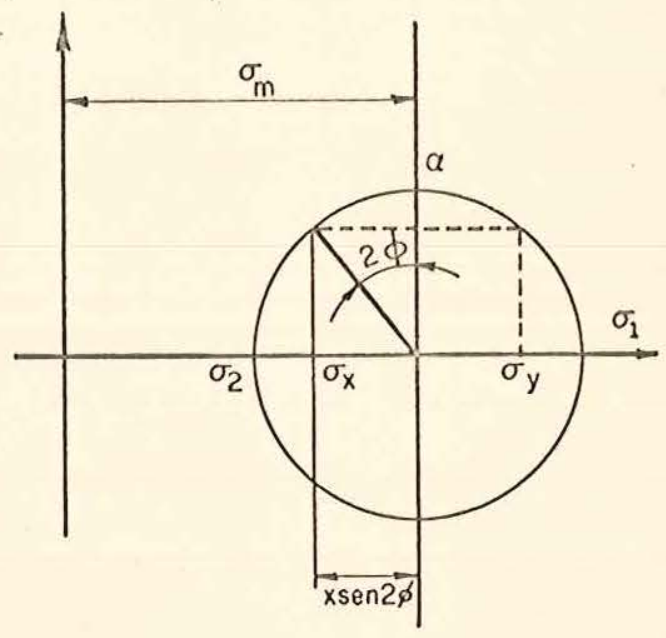

FIG. -4

Do círculo de Mohr da figura (4) representando o estado de tensões do ponto 0 da figura (5), teremos:

$$
\begin{aligned}
& \sigma_{x}=\sigma_{m}-k \operatorname{sen} 2 \phi \\
& \sigma_{y}=\sigma_{m}+k \operatorname{sen} 2 \phi \\
& \sigma_{x y}=\cos 2 \phi
\end{aligned}
$$

2.2- Estudo das tensões no ponto

Toma-se agora um ponto intersecção de uma linha de fluência com outras, conforme a figura (7) e seu círculo de Mohr correspondente, representado pela figura (6).

Seja então um ponto $P$ sob uma defornação plástica plana, este ponto está em um campo de linhas de fluência ( o pla no do papel representa o plano físico). Este ponto também é a ori gem de um sistema de coordenadas cartesianas, como mostrado na fi gura (7). 


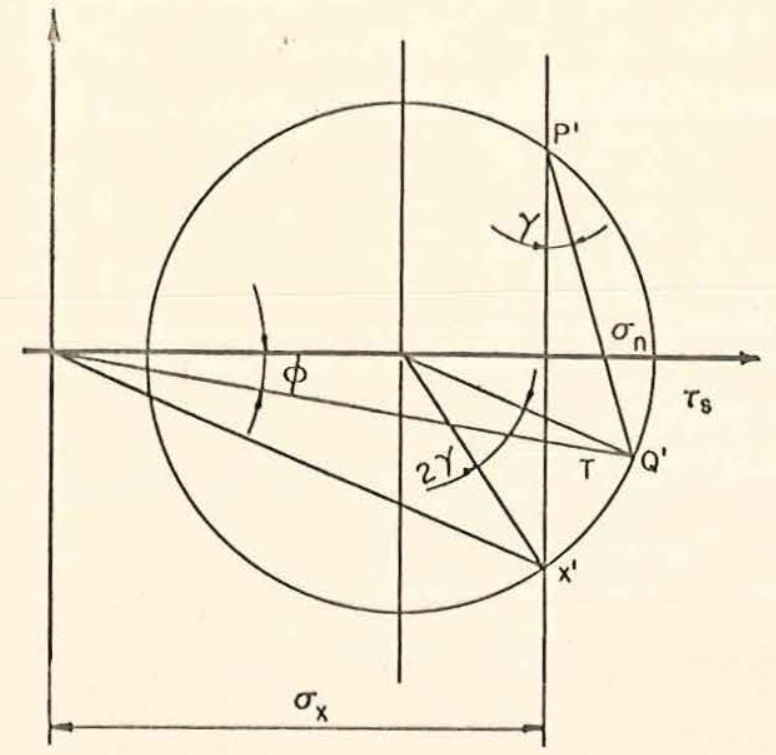

FIG. -6

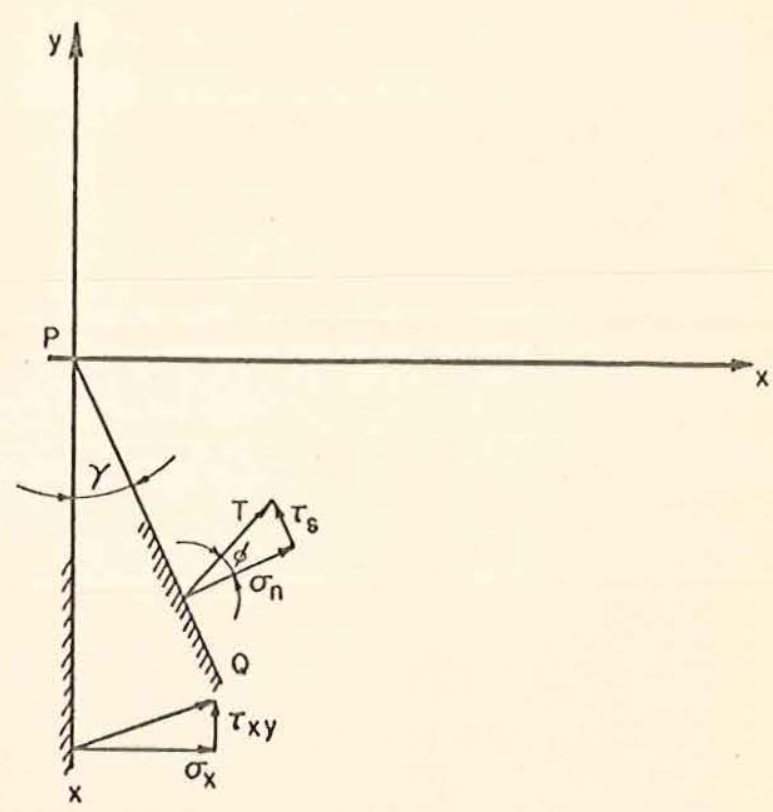

FIG.-7

Os ângulos, medidos no sentido antihorário, es tão relacionados não com as tensões, mas com os planos sobre os quais elas agem, в são contados a partir do ramo negativo do eixo dos y . As tensões atuantes no plano PX estão representadas pelo ponto $X '$ no círculo de fiohr da figura (6). Se considerarmos um plano PQ que fa ça um ângulo $\gamma^{\wedge}$ com o lado negativo do eixo dos $\gamma$, e neste ponto colocarmos uma tensão resultante $T$, esta tensão terá componentes $\tau_{S}$ e $\sigma_{\text {w }}$ que irão determinar o ponto $Q^{\prime}$ no círculo de Mohr. Para se deterninar a direção do vetor $T$, a normal $n$ do plano físico deve ser rodada de $\phi$, mas no sentido oposto. O diagrama de riobr resultanto é chamado de plano de tensões. 0 ângulo central que compreende o arco X' Q' é igual a $2 \gamma$ e medido no sentido antihorário a partir do raio que 
passa por $X^{\prime}$. As linhas $X^{\prime} P^{\prime}$ a $Q^{\prime} P^{\prime}$ são traçadas paralelas às $1 \underline{i}$ nhas $P X$ e formando assim un ângulo $\gamma_{\theta}$ con vertice em p', polo do círculo de mohr. Conhecido o polo, qualqquer outro ponto do círculo que corresponda a una superfície poderá determinar o sistema de ten sões descie que seja traçado a partir do polo, uma paralela à superfície dada. A interseção desta reta com o círculo correspondente a condição de tensões do elemento.

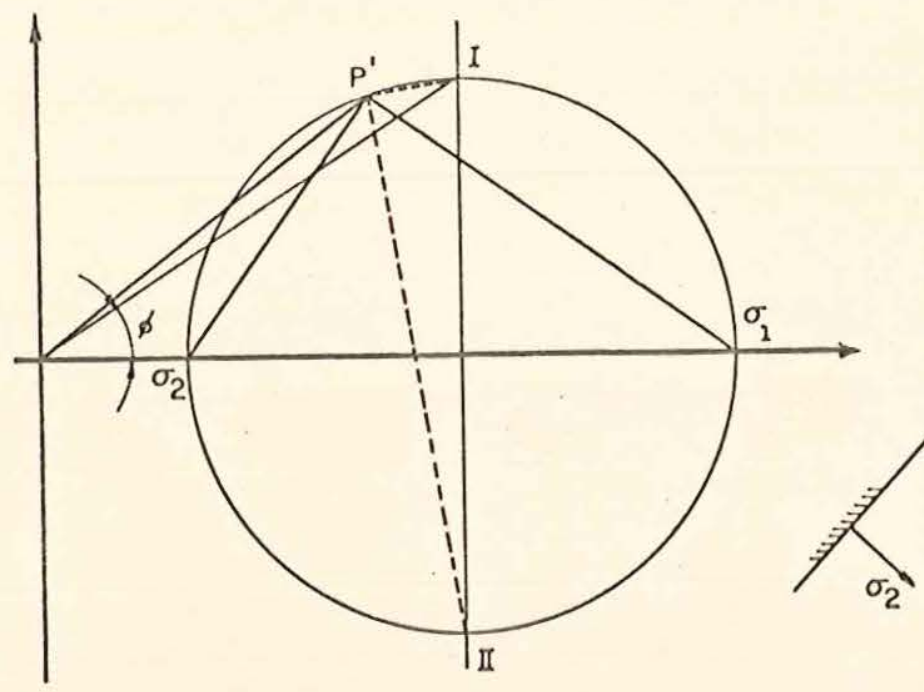

FIG. -8
FIG. -9

Os pontos I e II do círculo de Fohr da Pigura (8) a o correspondente plano físico representado pela figura (9), se repox ta`a elementos de superfície ondo se transmite a máxima tensão de cisą lhamento. As direções dos planos onde a tensão de cizalhamento é máxí ma são dadas por paralelas a p'I e prII e são chamadas de primeira e segunda tensão de cizalhamento correspondendo as linhas de fluência $\alpha$ e $\beta$.

2.3- Descontinuidade na tensão

Supõe-se agora que exista uma superfície en regi 
me de tração e que a resultante tonha um ângulo $\varnothing$ com a normal naquele ponto figuras (10) e (11).

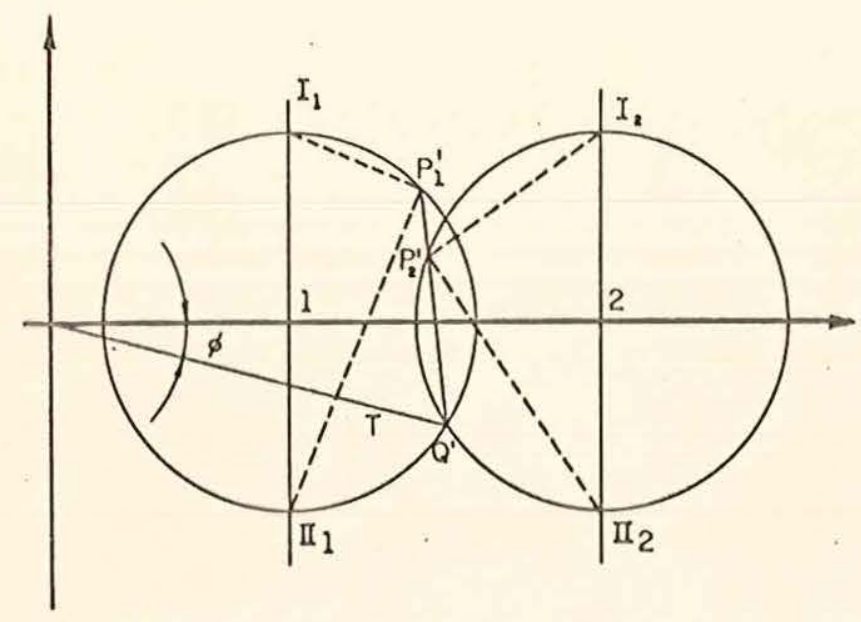

FIG. -10

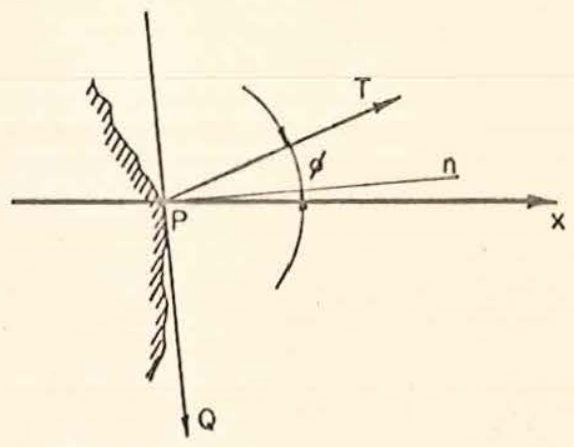

FIG.-1I

A tração $T$ e o ângulo $\not$ determinam o ponto $Q^{\prime}$ no círculo de fiohr que representa o estado de tensões no ponto P. Exis tem dois círculos com raio $\mathrm{K}$ que podem conter o ponto $\mathrm{Q}$, um com centro à direita e outro com centro à esquerda de $Q$. Q qualquer des tes círculos podem ser uma solução possível, se não tivermos outras condições de contorno. Esta incerteza vem do critério de resistência:

$$
\sigma_{x}-\sigma_{y}= \pm 2 \sqrt{k^{2}-\sigma_{x y}^{2}}
$$

Assim, se somente $\sigma x$ e 乙xy são conhecidos, existem dois valores para $\sigma_{y}$ (e com $\zeta_{x y}=0$, 0 valor máximo de $\sigma_{y}$ será função de $\pm 2 \sqrt{k^{2}}$ )

Existe então uma descontinuidade em uma das ten sões no porito P. Devido as condições de equilíbrio da figura (12), $\sigma x$ deve ser contínuo, mas $\sigma_{y}$ necessariamente não. 


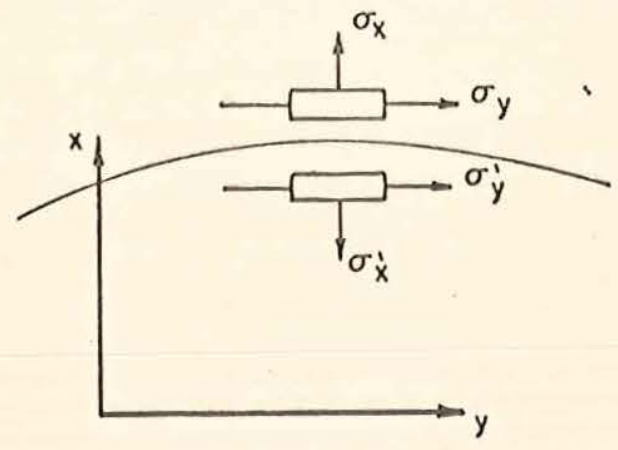

FIG. -12

2.4- Estudo de tensões ao longo da linha de fluência

Até aqui considerou-se o estudo de tensões em um ponto. Determina-se agora o estado de tensões quando nos movernos ao longo de uma linha de fl̆uência.

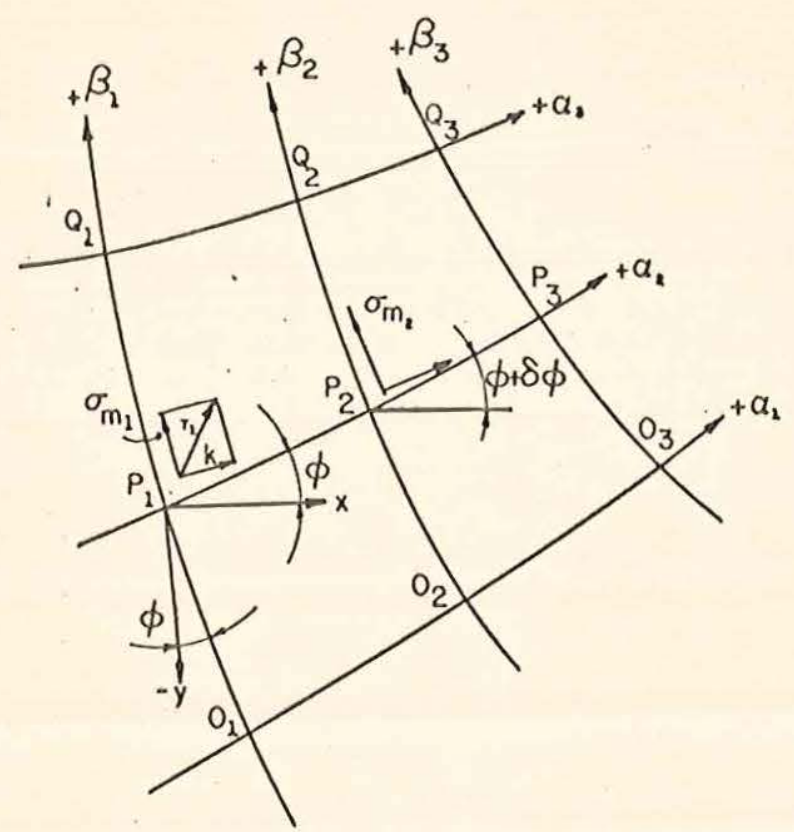

FIG. -13 
Seja a figura (13) representado o plano físico com Um campo de linhas de fluência nos quaís P1, P2, P3 ... são pontos de uma das linhas da família de curvas $\alpha$, e Q1, Q2, Q3 ... são os pontos de uma das linhas da família de curvas $\beta$. Supõe-se ainda que os ar cos de curvas P1, P2, P3 etc. possan ser substituídos por segmentos de retas (isto é, as distâncias $\mathrm{Pl}-\mathrm{P} 2, \mathrm{P} 2-\mathrm{P} 3$... são inuito pequenas) e que as direções entre o eixo $\mathrm{\theta}$ as linhas $\alpha$ formem ângulos positivos no sentido antihorário.

O estado de tensões é dado pelas equações (29) e (30) chamadas eq̣uações de Hencky

$$
\begin{aligned}
& \sigma m-2 k \phi=C_{\alpha}(1 \text { n'hos } \alpha) \\
& \left.\sigma m+2 k \phi=C_{\beta} \text { (linhal } \theta\right)
\end{aligned}
$$

Pela convenção de sinal adotada, as tensões de compressão são negativas e as de elongamento positivas. Na mesma con venção, as tensões surgem sobre os elementos achurados da figura (13).

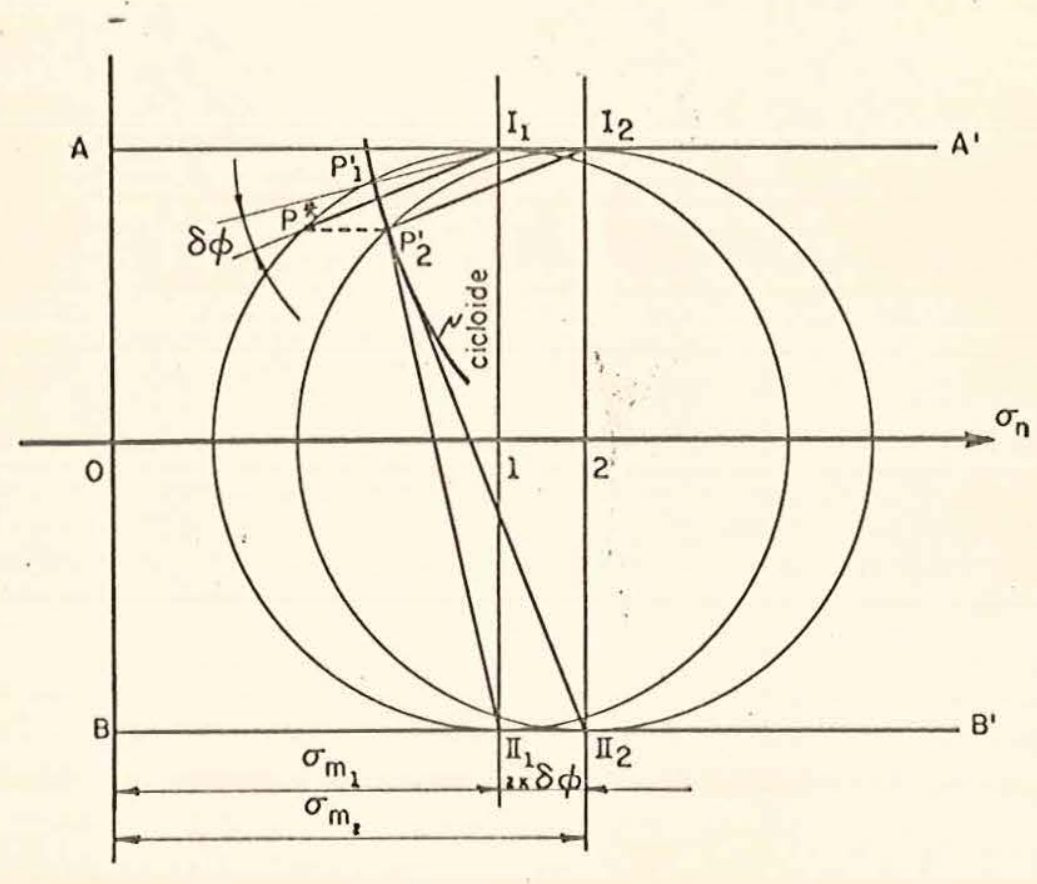

FIG. -14

0 vetor $T_{1}$ em $P_{1}$ dá o ponto $I$ da figura (14) 
Examina-se agora o que acontece ao círculo de Mohr ao longo da linha $\alpha$ quando a percorremos de Pl a P2. 0 estado de ten sões no ponto Pl é representado pelo círculo l da figura (14). Como foi visto, as linhas $I_{1}, P_{1}^{\prime}$ e II $I_{1}, P_{1}$ 'são paralelas às corresponden tes linhas de fluência peló ponto $P_{1}$ e dão o polo P'l. Das equações de Hencky, em Pl, temos:

$$
\sigma_{m 1}=c_{\alpha}+2 k \dot{\phi}_{1}
$$

в en $P 2$

$$
\sigma_{m_{2}}=c \alpha+2 k\left(\phi_{1}+\delta \phi\right)=\sigma_{m_{1}}+2 k \delta \phi
$$

0 estado de tensões no ponto P2 pode ser representado pelo 29 círculo de liohr a distância $\widetilde{\sigma} \mathrm{m}_{2}$ da origem e que pela nossa convenção de sinais, é consistente com o círculo traçado.

Novamente traçamos $I_{2} P_{2}^{\prime}$ e II $P_{2} P^{\prime}$ paralelas as $I \underline{i}$ nhas de fluência no ponto $P_{2}$, resultando o novo polo $P^{\prime}{ }^{\circ}$ As linhas $I_{2} P_{2}{ }^{\prime} I_{1} P^{\prime \prime}{ }_{1}$ formam un ângulo $\delta \varnothing$ entre si. Tracemos uma paralela a $\mathrm{I}_{2} \mathrm{P}_{2}$ por $\mathrm{I}_{1}$. Determinaremos o ponto $\mathrm{P}^{*}$ no círculo 1 . Devido ao fato que o ângulo no centro do círculo que subentende um arco é duas vezes maior àquele en cujo vértice está sua circunferência e suben tende o mesmo arco, 0 polo $\mathrm{P}_{2}$ é determinado por uma translação do círculo a uma distância $2 K \delta \varnothing$ e una rotação de um ângulo $2 \delta \varnothing$. Como o raio do círculo é $K$, isto é o mesmo que deslocarmos sem escor regamento o círculo $l$ ao longo da linha $A A^{\prime}$. Por este processo teremos então determinado o campo de tensões ao longo de urna linha $\alpha$. Da mesma maneira, pode-se ver que o campo de tensões ao longo de uma Winha $P$ estará determinado pelas ciclóides rolando ao longo da lí nha 83'. 0 arco $P_{1}{ }^{\prime} P_{2}$ 'é normal a linha $I_{1} P_{1}{ }^{\prime}$ no ponto $P_{1}{ }^{\prime}$. Como a linha $I_{1}, P^{\prime}$ é paralela a linha $P_{1} P_{2}$ do plano rísico, os segmentos de uma mesma linha de fluencia serão perpendiculares aos segmentos correspontentes da linha traçada no plano de tensões e a cada ciclóide(curva quo determina as tensões de uma linha de fluência) correspon de um valor de $C_{\alpha}$ ou $C_{\beta}$ constante. 
3- Estudo cinemático

3.1- Campo de velocidades

Determina-se agora o campo de velocidades das par tículas, en função das linhas de fluência.

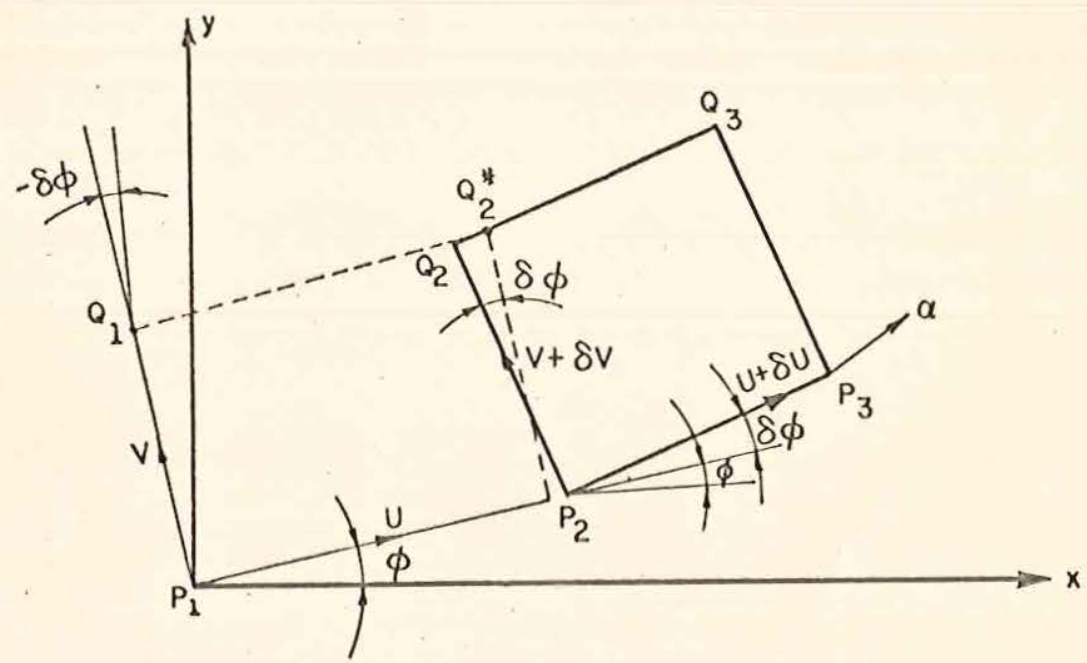

FIG.-15

Os segmentos de retas $P_{1}-P_{2} \quad P_{2}-P_{3}$, etc. fazem parte de uma linha $\propto$, como mostrado na figura (15), con ângulos $\not$, $\phi+\delta \not$, etc. no sontido antihorário a partix do oixo $\times$. A linha $\mathrm{P}_{2} \mathrm{Q}_{2}{ }_{2}$ é perpendicular a $\mathrm{P}_{1} \mathrm{P}_{2}$ e portanto un segmento de uma linha $\beta$. A velocidade do ponto $P_{1}$ tem componontes $\underline{u}$ e $\underline{v}$ nas direçues $\alpha$ e $\beta$ e a velocidade no ponto $P_{2}$ tem componentes $u+\delta u$ e $v+S v$ respecti vamente nas direções $\alpha$ e $\beta$. Para que não haja descontinuidade no ponto $P_{2}$, a projeção das velocidades neste ponto, na direção $P_{1} P_{2}$, deve ter como resultante $u$, assim:

$$
(u+\delta u) \cos \delta \phi-(v+\delta v) \operatorname{sen} \delta \phi=0
$$

e como $\delta \not$ é pequeno e desprezando os termos de 2 a ordem, temos:

$$
\delta v-v \delta \phi=0
$$


que no línite dá:

$$
\frac{\partial u}{\partial \phi}-v=0 \text { (linher } \alpha \text { ) }
$$

8 da mesma manoira:

$$
\frac{\partial v}{\partial \psi}+u=0(\text { nes linhes } \beta)
$$

As equações (39) e (40) são chamadas equações de Geiringer.

Prager mostrou como se constrói um diagrama de ve locidades ou hodógrafo, cori o uso da constância do volume do inate rial e das equações de Gejringer.

Vamos considerar o mesmo campo de linha de fluência mostrado na figura (13), e tomemos as componentes das velocidades nas direções $\alpha$ e $\beta$ como sendo $\underline{\underline{a}} \underline{\mathrm{v}}$ (fíjura 16 ).
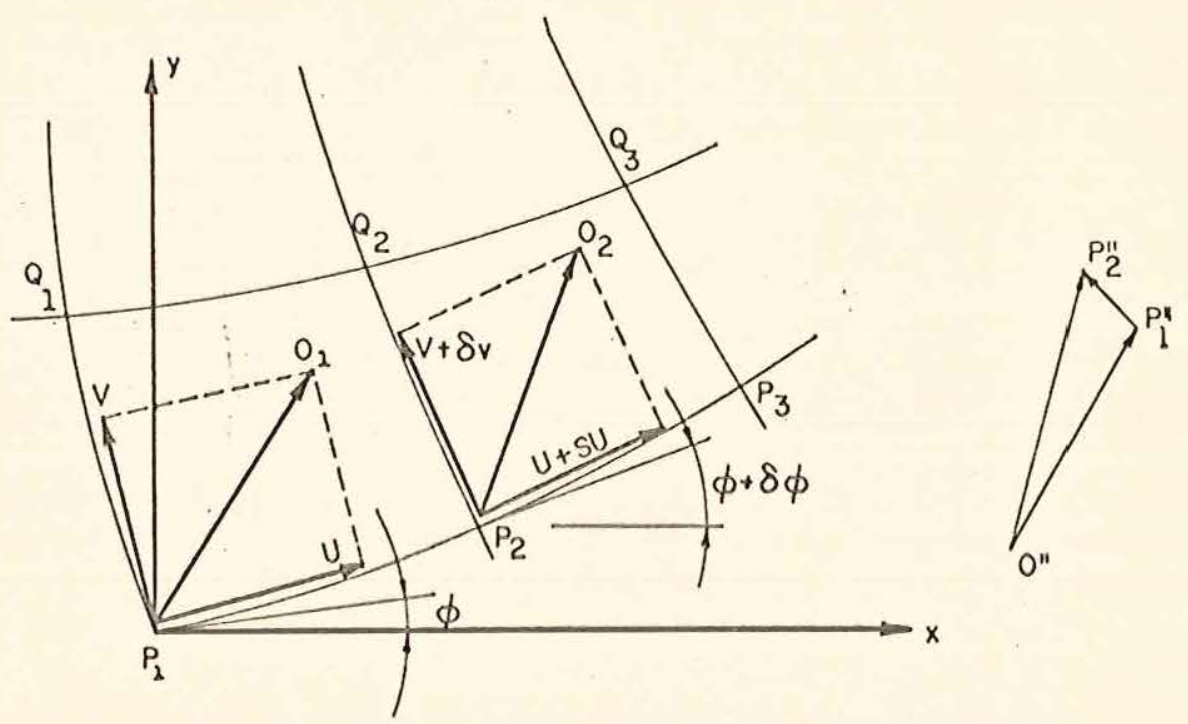

FIG. -16

A soma dos vetores $\underline{u}$ e $\underline{v}$ em $P_{\mathcal{J}}$ é a velocidade re sultinte $P_{1} \mathrm{O}_{1}$. Da mesma maneira a resultante em $P_{2}$ é $P_{2} 02$, soma 
de $u+\delta u$ e $v+\delta v$. Tomando una oxigem de velocidade 0 ' e traçado os vetores 0 ' $P_{1}$ ' rede de linhas de fluencia, a equacão de Geiringer Ju - $v \delta \phi=0$ diz que a projeção de $P_{1} \tilde{\prime}^{\prime} P_{2}{ }^{\prime \prime}$ sobro o elemento $P_{1} P_{2}$ deve ser zero, poi. $s$ $P_{1}{ }^{\prime} P_{2}{ }^{\prime \prime}$ é a diferença em velocidade de $P_{2}$ e $P_{1}$ a deve ser zoro na direção $P_{1} P_{2}$ para extensão nula da linha de rluência. Em outras pala vras, as linhas dos elementos do campo de velocidades devem ser nor mais as linhas correspondentes do elemento no campo físico. Em geral, os elementos correspondentes de um campo de linhas de fluência e o ho dógrafo são pexpendiculares entre si e os elementos correspondentes ao campo de tensões e o hodógrafo são paralelos entre si.

3.2- Descontinuidade na velocidadö

A incomprescibilidade do material nos diz que a componente normal da velocidade en uma linha de fluência deve ser con tínua, mas a componente tangencial, quando estamos em cada lado de uma linha de fluência, necessariamente não.

Como exemplo, vamos supor que a linha $A B$ da figu ra (17) seja parte de uma linha de fluência de una farnília $\propto$, e que nos pontos A a B tenhamos o cruzamento de outras linhas de flu彑 ência da família $\beta$ •

No ponto $A$, as componentes de velocidades nas di reções das linhas de fluência no lado 1 da curva $A B$ estão representa das, segundo a figura (17) por 뜨 e ‥ Se o material é contínuo e j.n compressível, a componente $v_{2}$ normal ao lado $A B$ deve ser igual a $v_{2}$, também normal a $A B$ o no lado 2 da figura (17). . Podemos visualiza o material do lado 2 deslizando sobre o material do lado 1 paralelo a linha de fluência em $A$, e isto pode ser indicado vetorialmente por $\mathrm{u}_{2}$ diferente de $u_{1}$ figura (18)。

$R_{1}$ é a velocidade resultante no lado 1 e $R_{2}$ é a velocidade resultante no lado 2 , com uma descontinuidade $\left(u_{2}-u_{l}\right)$ re lativa ao lado l. 0 vetor $u_{2}-u_{1}$ deve ser tangencial a linha de flu ência em $A$. 


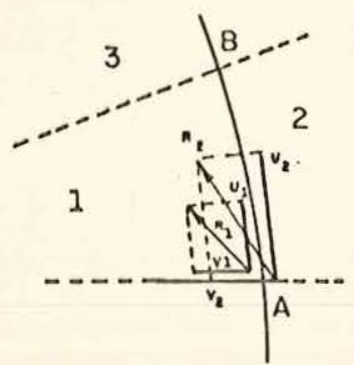

FIG -17

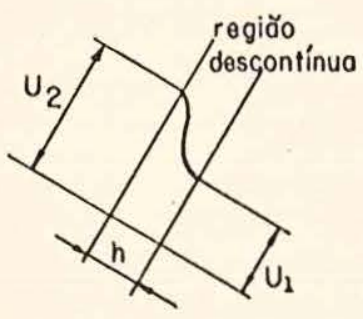

FIG.-19

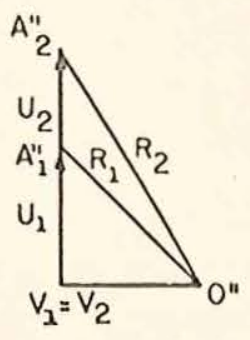

FIG. -18

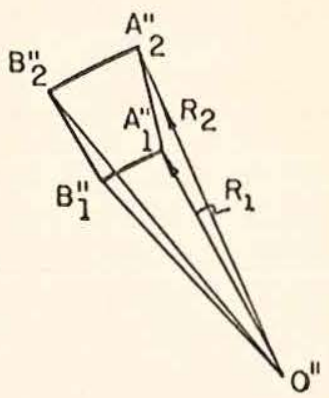

FIG -20

Pola figura (19) podemos considerar a descontinui dade no campo de velocidades como uma faixa muito pequena no plano fí sico, onde a velocidade varia rapidamente de una maneira contínua. Se h tende a zero a razão de cisalhamento tende a infinito. Referindo- se novamente à figura (17), as equações de Geiringer para o ponto A es tabelecem para o lado 1.

$$
\delta v_{1}-v_{1} \delta \phi=0
$$

e para o lado 2

$$
S v_{2}-v_{2} \delta \phi=0
$$


e como $v_{1}=v_{2}$, temos:

$$
\frac{d}{d \phi}\left(u_{2}-u_{1}\right)=0
$$

ou $u_{2}-u_{1}=$ cte

Ao longo de uma linha de descontinuidade, o sal to na componente tangencial é constante nesta linha. o hocógrafo a gora pode ser completado para o elemento AB, conforme a figura (20). Os pontos $0^{\prime \prime}$, A'' ${ }_{1}$ e $A^{\prime \prime}{ }_{2}$ rorman o diagrama de velocidades do pon to $A$. 0 vetor $0{ }^{\prime \prime} B^{\prime \prime}$, representa a velocidade do material inodiata mente no canto do setor 3 , determinado pelas equarões do Geiringer e o conhecimento do ângulo $\not$ ontre $A$ e B, pela linha AB. A equação(44) nos diz que $B^{\prime \prime}{ }_{1} B^{\prime \prime}{ }_{2}=A^{\prime \prime}{ }_{1} A^{\prime \prime}{ }_{2}$ e $B^{\prime \prime}{ }_{1} B^{\prime \prime}{ }_{2}$ é ortogonal a $A B$. qualquer ponto da curva $A^{\prime \prime}$ I. B' 1 rolativo ao ponto rixo G'' representa a velocidade do ponto correspondente en $A B$ do lado esquerdo, da mesma maneira, qualquer ponto de $A^{\prime \prime}{ }_{2} B^{\prime \prime}{ }_{2}$ relativo ao ponto 0 '' representa a velocidade correspondente do ponto no lado direito de A3. As curvas são paralelas.

4- Teorema de Hencky

Considera-se agora o elemento $A B P Q$ de uma malha de linhas de fluência $\alpha$ a $\beta$ mostrado na figura (21).

Das equações (29) e (30) têm-se que a diferença entre as tensões médias $\mathcal{V}_{m}$ calculada entre $A$ e $Q$, será pelo ponto $B$ :

$$
\sigma_{Q}-\sigma_{A}=\left(\sigma_{Q}-\sigma_{B}\right)+\left(\sigma_{B}-\sigma_{A}\right)
$$

e também

$$
\sigma_{Q}-\sigma_{A}=2 k\left(\phi_{Q}-\phi_{B}\right)+2 k\left(\phi_{A}-\phi_{B}\right)=2 k\left(\phi_{Q}+\phi_{A}-2 \phi_{B}\right)(46)
$$

Da mesma maneira pelo ponto P será:

$$
\begin{aligned}
\sigma_{Q}-\sigma_{A} & =\left(\sigma_{Q}-\sigma_{P}\right)+\left(\sigma_{P}-\sigma_{A}\right)=2 k\left(\phi_{P}-\phi_{Q}\right)+2 k\left(\phi_{P}-\phi_{A}\right)= \\
& =2 k\left(2 \phi_{P}-\phi_{Q}-\phi_{A}\right)
\end{aligned}
$$




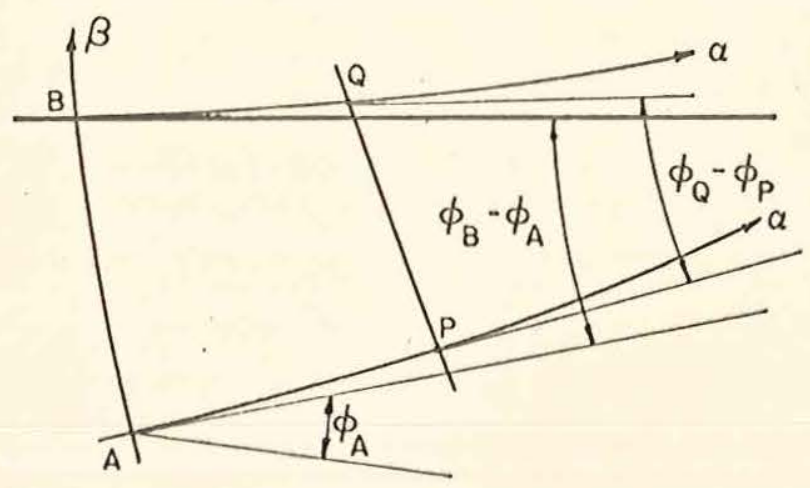

FIG.- 21

pela igualdade das equações (46) \& (47) temos:

$$
2 \phi_{P}-\phi_{Q}-\phi_{A}=\phi_{Q}+\phi_{A}-2 \phi_{B}
$$

ou

$$
\phi_{Q}-\phi_{P}=\phi_{B}-\phi_{A}
$$

isto é, " o ângulo entre duas linhas de fluência de uma mesma famália permanece constante nos pontos de intorseção com as linhas da outra família" (Henckyl.

5- Aplicação do método

5.1- Determinação dos pontos intersecção das linhas

A partir deste teorema pode-se determinas os pontos de intersecção das linhas de fluencia pelas interseções de segmentos de retas. Para tanto, vamos considerar o caso de forjamen to gom ferramenta reta e paralela a face a deformar.

No caso da operação de forjamento, o material imediatamente abaixo da ferramenta sofre uma deformação homogenea • A região homogenea fica definida por duas rolas, a primeira, saindo do canto díreito da ferramenta, que pertence à família de curvas $\beta$, 
faz um ângulo $\not$ a 1359 com o eixo $\times$, que estamos supondo perpendicular ao eixo da rerramonta, como mostrado na figura (22). 0 sistena de coor denadas adotado é o cartesiano com o eixo $x$ positivo na direção $A B$ e partindo do A para B, a origem colocada sobre o ponto $A$ e o eixo y com a direção perpendicular a $\times$ e sentido de cima para baixo.

A segunda reta, saindo do canto esquerdo da fer ramenta, pertence à família de curva $\alpha$ e faz um ângulo $\varnothing=1350$ com o eixo $y$, suposto na direção do eixo da ferranenta, como mostrada na figura (22).

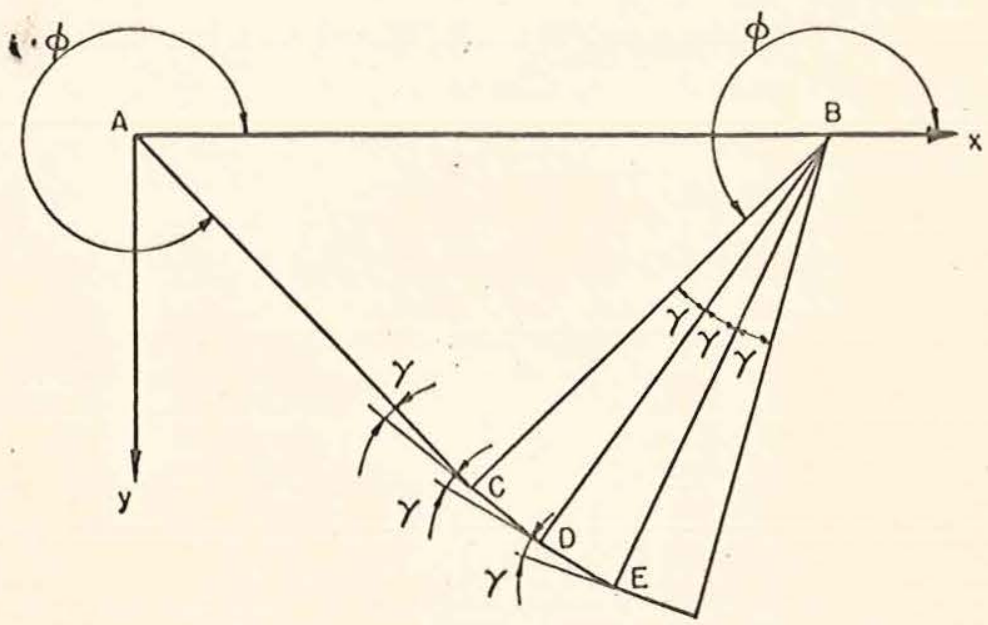

FIG. -22

como seguat

Podemos construir o restante das linhas $<$ e 3 , A partix do ponto fै irigura (22), tragaromos um

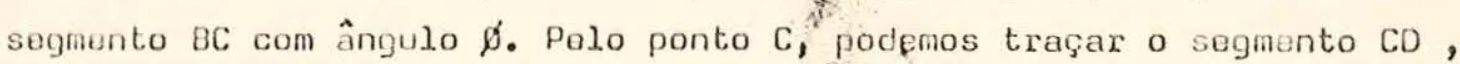
formando um ângulo com o segmento AC. Segundo o teorema de Hencky os segmentos $8 D$ a $C D$ pertencem às famílias de curva $\alpha$ e $\beta$ ortogonais. Os pontos $C, D, E$, etc, pertencem a um arço de círculo com centro em $B$ e raio $B C$. Os arcos (cordas) CD, DE, etc. pertencem à prineira cur va da família $\beta$ в os raios $B C, B D, B E$, etc. pertencem respectivamen 
te às 1a, 2a, 3a, otc curvas da família $\alpha$. Numerando as curvas a par tir do ponto $\mathrm{C}$ (este ponto pertence à primeira curva da família $\alpha$ e à primeira da família $\beta$ o porsimetria, teremos o conjunto de linha s e pontos oomo mostrado na figura (23)

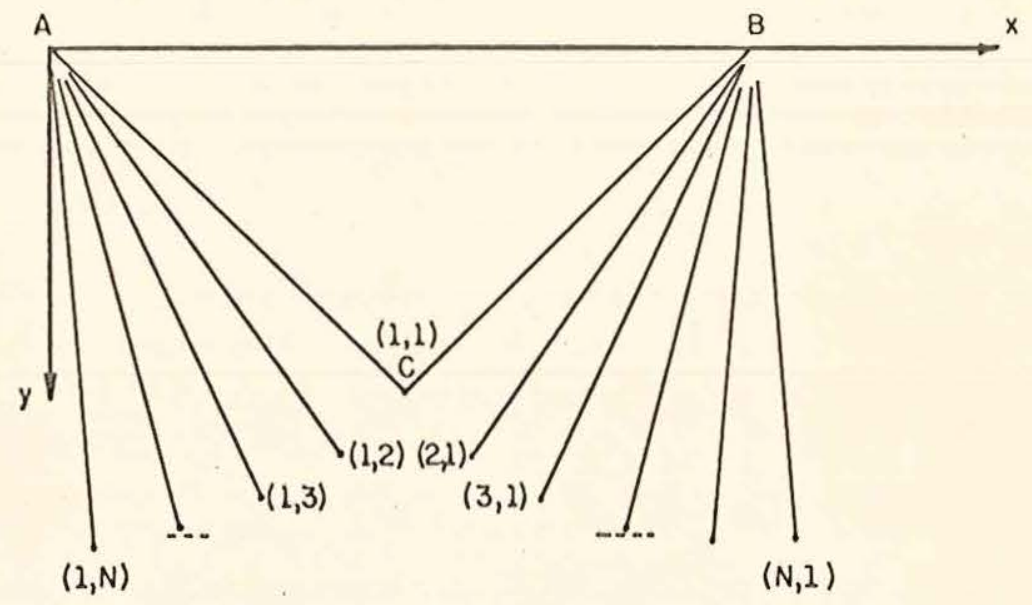

FIG. -23

Aplicando novamente o teorema de Hencky aos pon tos: $(1,2),(1,1)$ e $(2,1)$, podemos determinar o ponto $(2,2)$ como mos trado na figura (24).

Pola figura (24) ternos já determinados:

$\left(x_{C}, Y_{C}\right)=\operatorname{ponto}(1,1)$

pontos $D\left(X_{d}, Y_{d}\right) \quad E$ ponto $(2,1)$

$F\left(X_{f}, Y_{f}\right)=$ ponto $(1,2)$

ângulos

$$
\theta=45^{\circ}+\gamma-\gamma=45^{\circ}
$$

$$
\Delta=45^{\circ}+\gamma-\gamma=45^{\circ}
$$

e não conhecemos, as coordenadas do ponto $(2,2)$, isto é, $\left(x_{h}, y_{h}\right)$. 


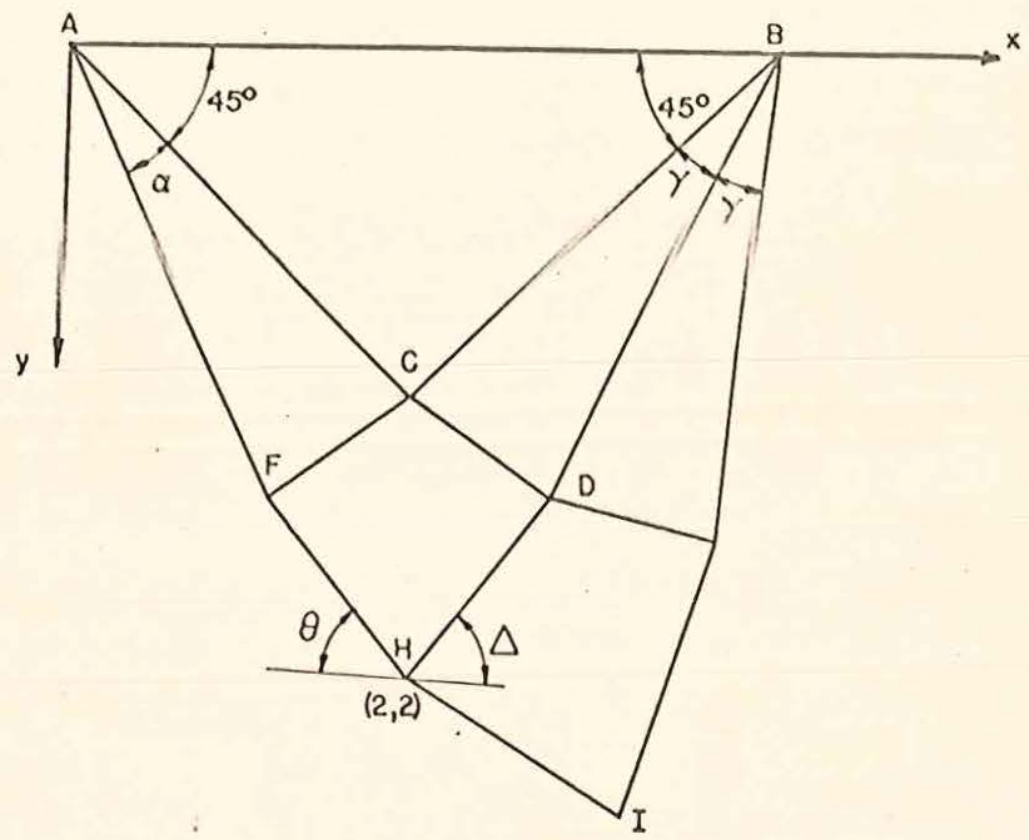

FIG. -24

Este ponto pode ser determinado pela interseç̧ão das retas que passarn polos pontos $\mathrm{FH}$ в DH. Podemos então, conhecidas as coordenadas do ponto $H$, deterninar de maneira semelhante as do ponto I, assim sucessivamente.

A sequência do cálculo fica sssin constiluída : A partir dos pontos A é B ( a distência $A B$ é igual ao com rimento da ferramenta) e com um sistema $x y$ de coordenadas como indicado na $f \underline{i}$ gura (22), teremos:

$$
\begin{aligned}
& J=1,2, \ldots \ldots N \\
& A B=b \\
& x(1, J)=b-\frac{b}{\sqrt{2}} \cos [45+(J-1) \gamma]
\end{aligned}
$$

linha 3

$$
y(1, J)=\frac{b}{\sqrt{2}} \quad \operatorname{sen}[45+(J-1) \gamma]
$$


$I=1,2 \ldots \ldots N$
$x(I, I)=\frac{\mathrm{b}}{\sqrt{2}} \cos [45+(I-1) \gamma]$

linha $\alpha_{1}$

$Y(I, I)=Y(I, J)$
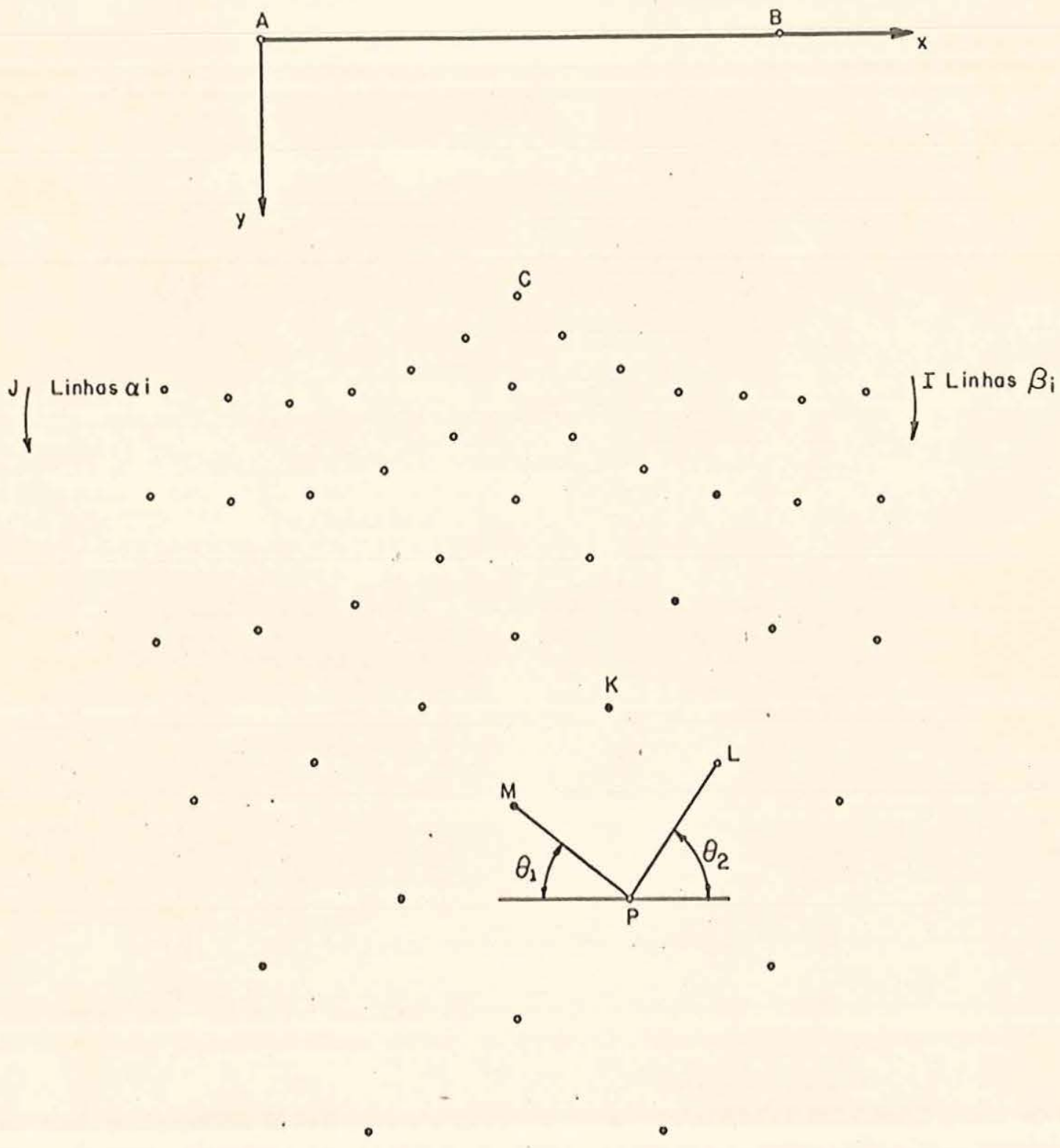

$(N, N)$ 
Os ponitos $(I, 1)$. IEl, $N$ da linha $\alpha_{1}$ e os pontos $(1, J)$, $J=l, \quad N$ da linha $P$ ficam determinados. Qualquer outro ponto pocierá ser determinado da seguinte maneira:

Estamos sup̣ondo a numeração indicada na figura (25), para os pontos, is to $\dot{\theta}$ :

As linhas $\beta_{i}$ e $\alpha_{i}$ recobem numeração crescente no sentido do triângulo $A B C$ para o ponto $(N, N)$

$\begin{array}{cl}\text { ponto } k & \times(I, J) \\ & y(I, J) \\ \text { pponto } m & x(I+1,3) \\ & y(I+1, J)\end{array}$

ponto $L \quad X(I, J+I)$

$y(I, J+1)$

ponto $P$

$x(x+1,3+1)$

$y(I+1, J+1)$

ângulos

$$
\theta_{1}=4 \dot{5}+(I-J) \gamma
$$

$$
\theta_{2}=45+(J-I) \gamma
$$

B

$$
\begin{aligned}
& x(I+1, J+1)= {[Y(I, J+1)-Y(I+1, J)-x(I+1, J)] t_{y} \dot{\theta}_{1}+} \\
&\left.\quad+x(I, J+1) \operatorname{tg} \theta_{2}\right] /\left(\operatorname{tg} \theta_{1}+\operatorname{tg} \theta_{2}\right) \\
& Y(I+1, J+1)= {[X(I+1, J+1)-x(I+1, J)] \operatorname{tg} \theta_{1}+Y(I+1, J) } \\
& \not(I, J)=\theta_{2}
\end{aligned}
$$

A partir dos pontos $(I, J)$ a $(I, I)$ podemos calcular todos os outros pontos com as expressões acima.

Determinaremos assim os pontos das linhas $\alpha_{i}$ e $\beta_{i}$ perpen diculares entre si e onde valem as expressões acima.

Como condição de contorno; pelo pontio $(N, N)$, junção das linhas $\alpha_{N}$ e $\beta_{N}$, passam duas tangentes às curvas $\alpha_{N}$ e $\beta_{N}$ que fazem un ângulo igual a 450 como o eixo $x$.

É conveniente fazermos uma translag̃ão do sistema de coor denadas para que o ponto $(N, N)$ coincida com a origem e os eixos $x$ e y te nham as orientações normais indicadas na literatura. Para tanto podemes fa zer: 
A B

。
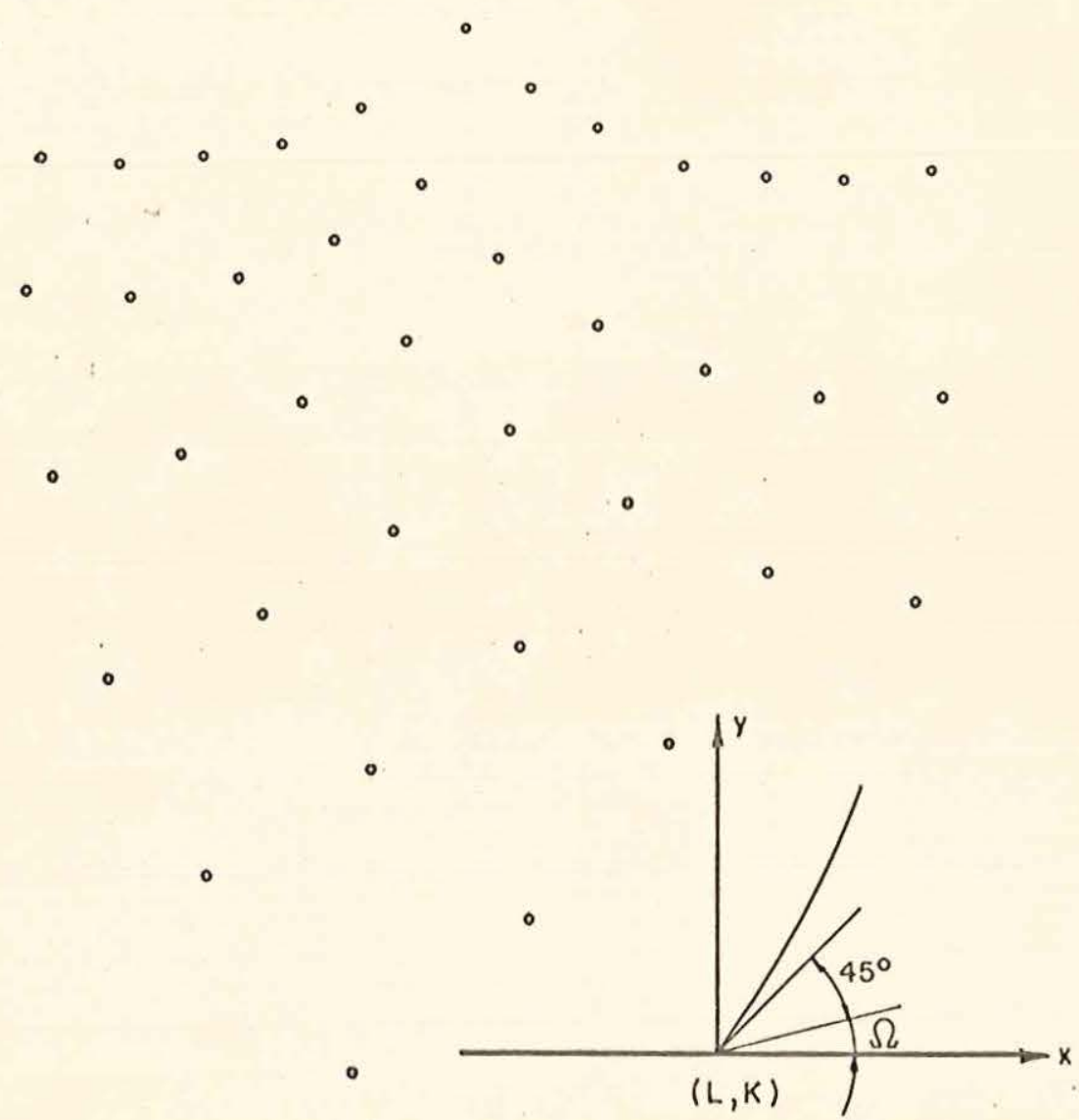

$0(N, N)$

FIG. -26 


$$
\begin{aligned}
& Y^{\prime}(I, J)=Y(N, N)-Y(I, J) \\
& X^{\prime}(I, J)=X(I, J)-X(N, N)
\end{aligned}
$$

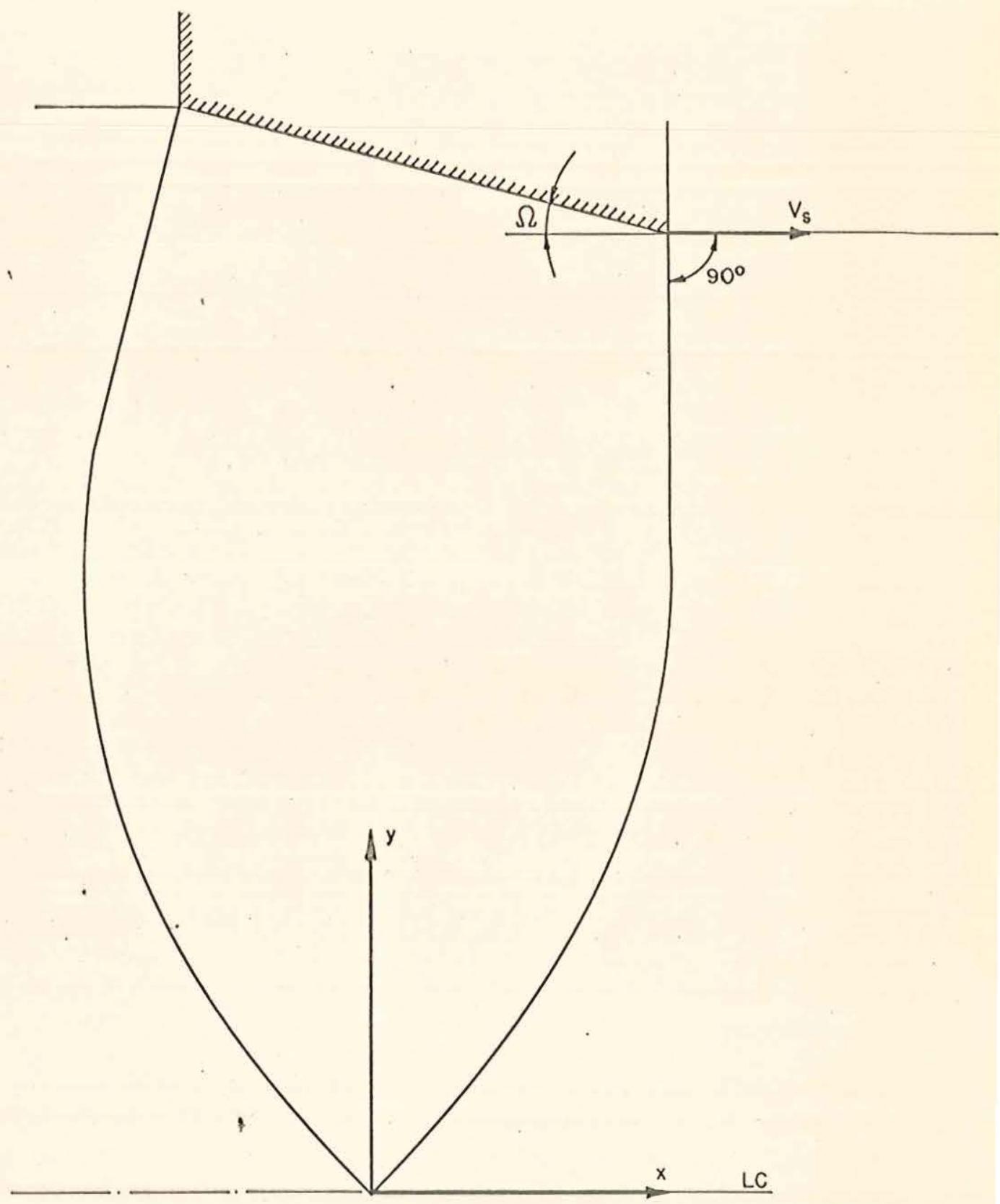

FIG. -27 
Para manter esta condição de contorno expressa pg las equações (61) a (62) nos caecsdo trệilação, \&̊ necessárío que so escoliha uma linha particular do tal modo que sua tangente no ponto om quo so encontra con a linha $\alpha$ tenha um ângulo igual a $45 \Omega$ com rolação so eixo $X_{0}$

Cum estas imposiçõos, a nossa oxigom soxả dosloca da para o ponto $\left(L_{0} K\right)$ como mostrado na figura (26).

A condição do quo as linhas $\alpha$ e $\beta$ se cruzam na orignn pormenco ênjulos do 45 con o oíxo $X$ podes ser obtida pos uma rotiação do um ângulo iqual ao da fỉsíra, cono mostracio na pigura (27).

As вqunçöos corrospendenius para a novo sistoma do coordonadas sorãos

$$
\begin{aligned}
& X(X, J)=Y\left(Y_{0} J\right) \operatorname{son} \Omega+X\left(X_{0} J\right) \cos \Omega \\
& Y(I, J)=\gamma\left(I_{0} J\right) \cos \Omega-X\left(X_{0} J\right) \operatorname{sen} \Omega
\end{aligned}
$$

Nos casos do extrusäo o porjanento con natríz Pe

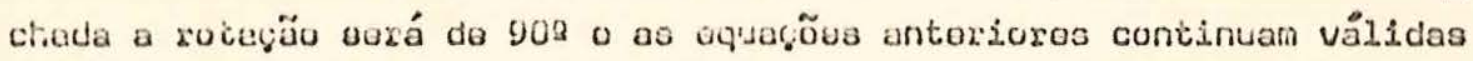
com a oxizea colocada no ponto (Hoiv).

Com os vários pontos das linhas, pociomos agora colcliar as éreas parciajo nas dirogõos $\mathrm{X}$ a $\mathrm{Y}$.

Ároas nas díroģõos $X$ \& $Y$

Linhas

$$
\begin{aligned}
& A_{X}=Y^{2}\left(I_{0}, 3\right)-Y^{2}\left(x_{0}, 3\right) \\
& A_{Y}=X\left(I_{0} J\right)-X\left(x_{3} 3\right)
\end{aligned}
$$

Linkas

$$
\begin{aligned}
& A_{X}=Y^{2}\left(r_{0} J\right)-Y^{2}\left(I I_{0} J\right) \\
& A_{y} \equiv X\left(I_{0} J\right)-X\left(I_{0} J\right)
\end{aligned}
$$

Tomos assin as tonsões nas direções $X$ o $Y$ calcuLodes polas expressôos (31)。(32)。(33)。(29)。(30)。 es áreas polas exprossōos (65)。(66), (67) e (68) o podomos ontão calcular a épça 
em cada trocho.

Das equar,ões (31), (32), (33), (29) a (30) aplica das ao ponto $(1,1)$ temos:

$$
\sigma_{x(1,1)}=0
$$

que substituida na (3.1):

$$
\begin{array}{r}
\sigma_{m}(1,1)=k \operatorname{sen}(2 \phi(1,1)) \\
c_{2}(1)=k[\operatorname{sen} 2 \phi(1,1)+2 \phi(1,1)]
\end{array}
$$

como $C_{2}$ (1) é constante ao longo da linha $\beta_{1}$ termos: no ponto $(1, i)$

alén disoo

$$
\sigma_{m}(1, N)+2 k \phi \varnothing(1, N)=C_{2}(N)
$$

$$
\sigma_{m}(1, N)-2 k \quad \not f(1, N)=C_{1}(N)
$$

a solução dio $(72)$ e $(73)$ dará o valor de $\sigma_{m}(1, N)$ e $c_{1}$ (N)

$$
\begin{aligned}
& c_{1}(N)=k[4 \phi(1, N)+\operatorname{sen} 2 \phi(1,1)+2 \phi(1,1)] \\
& \sigma_{m}(1, N)=c_{1}(N)-2 k \phi(1, N) \\
& \sigma_{x}(1, N)=\sigma_{m}(1, N)-k \operatorname{sen} 2 \phi(1, N) \\
& \sigma_{y}(1, N)=\sigma_{m}(1, N)+k \operatorname{sen} 2 \phi(1, N) \\
& \sigma_{x y}(1, N)=k \cos 2 \phi(1, N)
\end{aligned}
$$

como $C_{1}(n)$ é constante ao longo da linha $\alpha_{N}$, teremos para locios os pontos desta linha:

$$
\begin{aligned}
& \sigma_{m}(I, i)=c_{I}(i)+2 k \phi(I, N) \\
& \sigma_{x}(I, N)=\sigma_{m}(I, i)-k \operatorname{sen} 2 \phi(I, N) \\
& \sigma_{y}(I, i)=\sigma_{m}(I, N)+k \operatorname{sen} 2 \phi(I, N) \\
& \sigma_{x y}(I, N)=k \cos 2 \phi(I, N)
\end{aligned}
$$

A força em cada trecho e nas diregões $x$ e $y$ será, respectivamente o prodiuto da (80) pela (65) ou (67) e da (81) pe la (66) ou $(68)$.

A soma das forjas elementares ao longo de uma 
IInha ou segundo a direção $X$ ou $Y$ será a resultante nesta mesma dirração.

5.2- Hodógrafo

0 hodógrafo pode ser determinado pela condição de perpendicularidade entre os elementos correspondentes, como já de monstrado. Este perpendicularismo poder ser conseguido pela image m inversa dos pontos com relação ao eixo Y figura (28).

Além da inversão da imagem em relação ao eixo $Y$, é necessário uma troca dos indices $X$ a $J$ que depinem as linhas $\chi$ o $\beta$ a uma mudança do ponto origem (o ponto $(1,1)$ passará a ser $(L, K)$ para trefilação ou $(N, N)$ para forjamento).

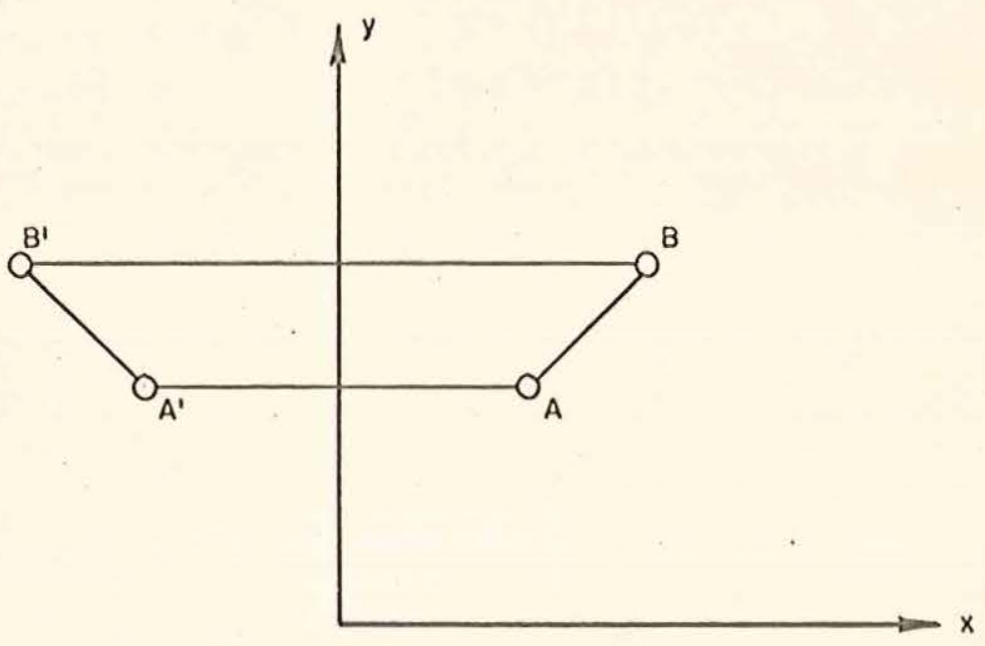

FIG. -28

5.3- Interpolação de pontos

Queremos agora determinar os ângulos das linhas de cisalhamento máxímo em um ponto $P$ que não pertença as linhas já determinadas. Para isso, vamos determinar o ponto 0 da figura (29), intersecção das retas que passam por $D, A, \theta C, 8$, pontos das linhas de pluêncía já determinadas. 


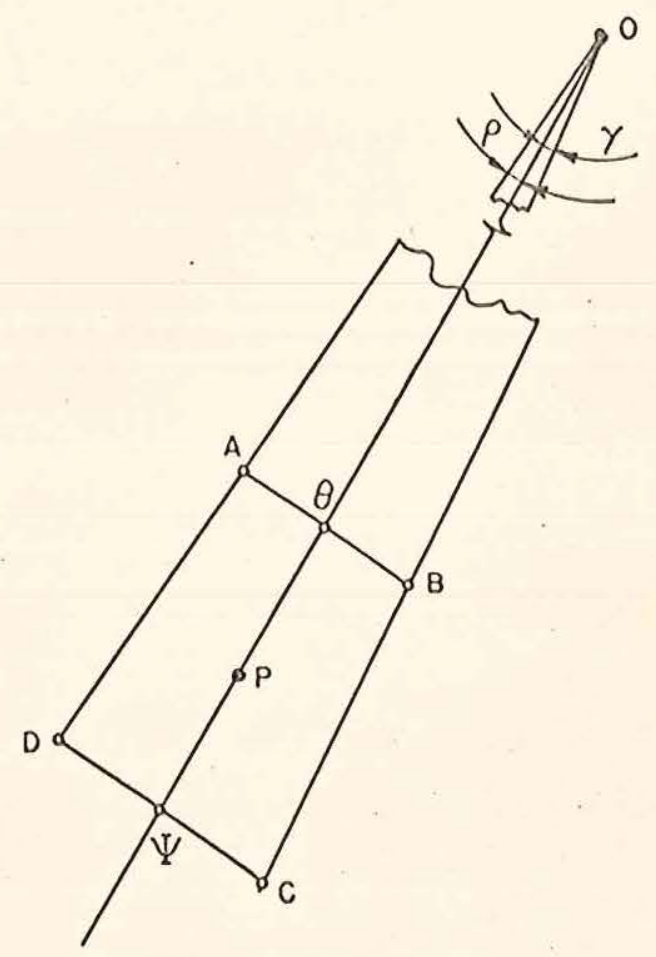

FIG. -29

Supondo que a reta $A B$ seja a corda de um arco $A B$ com centro em o e raio $r \simeq r_{A} \simeq r_{B}$, a que a reta $D C$ seja também a cor da cle un arco DC com centro em 0 , podemos traçar a reta op que irá in terceptar as retas AB e CD nos pontos $\theta$ e $\psi$, respectivamente.

A relação entre $A O$ e $A B$ é proporcional a relação en tre $\rho$ e $\gamma$, sendo $\gamma$ o ângulo incremento para o traçado das linhas de ruvencia, e $P$ o ângulo incremento da. Iinha de fluência que pas sa pelo ponto $P$. A relação entre $\theta P$ e $\theta \psi$ ó proporcional a rulação entre $\rho$ e $\gamma$, sendo $\rho$ o ângulo incremento da linha de fluéncia que passa pelo ponto $P$.

Dotorminados este ângulos, podemos, pelo teorema de 
Hencky, traçar as linhas $\alpha \beta \beta$ que passam pelo ponto $P$ de maneira idên ti.ca a utilizada para a deterninação dos pontos da malha.

Tendo as coordenadas dos pontos deformados no ma terial, podemos, com a colocação destes pontos no gráfico oriuncio da passagem do programa pelo computador, determinar as linhas I e $J$ majs próximas do ponto. Entrando com as coordenadas dos pontos o as li nhas I o $J$ mais próximas, teremos os pontos $A, B, C$ e $D$ com coordena das respectivamente iguais a $(X(I, J), Y(I, J)),(X(I+1, J)$, $(Y(I+1, J)),(X(I+1, J+1), Y(I+1, J+1))$ e $(X(X, J+1), Y(I, J+1))$

0 ponto 0 terá coorcienadas, com

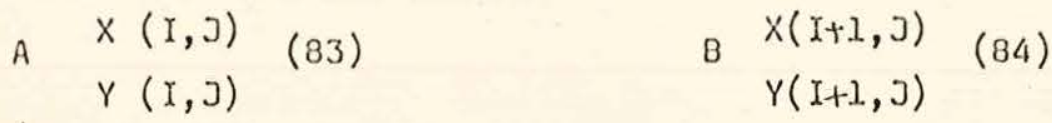

$$
\begin{aligned}
& \text { C } \begin{array}{l}
X(1, J+1) \\
Y(I 1, J+1)
\end{array} \\
& \text { D } \begin{array}{l}
X(I, J+1) \\
Y(I, J+1)
\end{array} \\
& Y_{P}=\frac{\left(x_{B} Y_{C}-X_{C} Y_{B}\right)\left(Y_{A}-Y_{D}\right)-\left(X_{A} Y_{D}-X_{D} Y_{A}\right)\left(Y_{B}-Y_{C}\right)}{\left(Y_{B}-Y_{C}\right)\left(X_{D}-x_{A}\right)-\left(X_{C}-x_{B}\right)\left(Y_{A}-Y_{D}\right)} \\
& X_{P}=\frac{Y_{P}\left(X_{D}-X_{A}\right)+X_{A} Y_{D}-X_{D} Y_{A}}{Y_{D}-Y_{A}}
\end{aligned}
$$

0 ponto $\theta$ terá coordenadas, com ponto lído $X, Y$

$$
Y_{\theta}=\frac{\left(Y_{D}-Y_{C}\right)\left(X_{P} Y-X_{P}\right)-\left(Y_{P}-Y\right)\left(X_{D} Y_{C}-X_{C} Y_{D}\right)}{\left(X_{C}-X_{D}\right)\left(Y_{P}-Y\right)-\left(Y_{D}-Y_{C}\right)\left(X-X_{P}\right)}
$$

$$
x_{\theta}=\frac{Y_{\theta}\left(X-X_{p}\right)+X_{p} Y-X Y_{p}}{Y-Y_{p}}
$$

e o ponto $\psi$

$$
Y_{\Psi}=\frac{\left(X_{A} Y_{B}-X_{B} Y_{A}\right)\left(Y_{P}-Y\right)-\left(X_{P} Y-X_{P}\right)\left(Y_{A}-Y_{B}\right)}{\left(X-X_{P}\right)\left(Y_{A}-Y_{B}\right)-\left(X_{B}-X_{A}\right)\left(Y_{P}-Y\right)}
$$




$$
X_{Y}=\frac{\left(X_{A} Y_{B}-X_{B} Y_{A}\right)-Y_{Y}\left(X_{B}-X_{A}\right)}{Y_{B}-Y_{A}}
$$

Os pontos da linha $\beta$ que passam pelo ponto $(X, Y)$, pelo teorema de Hencky, terão as coordenadas:

$$
\begin{aligned}
& X(J)=\left(X_{\theta}-X_{A}\right)(X(I+1, J)) /\left(X_{B}-X_{A}\right)+X(I, J) \\
& Y(J)=\left(Y_{\theta}-Y_{A}\right)(Y(I+1, J)-Y(I, J)) /\left(Y_{B}-Y_{A}\right)+Y(I, J)
\end{aligned}
$$

os pontos da linhas $\alpha$ que passam pelo ponto $(X, Y)$, peìo mesmo te orema, Łerão as coordenadas:

$$
\begin{aligned}
& X(I)=\left(X-X_{\theta}\right) \quad(X(I+I, J)-X(I, J)) /\left(X_{\Psi}-X_{\theta}\right)+X(I, J) \\
& Y(I)=\left(Y-Y_{\dot{\theta}}\right) \quad(Y(I+I, J)-Y(I, J)) /\left(Y_{\Psi}-X \theta\right)+Y(I, J)
\end{aligned}
$$

Com L a M, respectivamente I,J, o número das lintias $\alpha$ e $\beta$ menores mais próximos do ponto lido, teremos pa ra o hodógrafo, segundo a rigura (30).

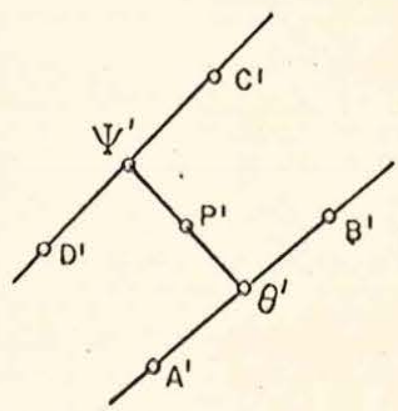

FIG. -30

e os pontos $\theta^{\prime}, \psi^{\prime}$ e p' ficarão com coordenadas:

$$
\frac{Y_{B}-Y_{A}}{Y_{B}{ }_{B}-Y^{\prime}{ }_{A}} \cdot\left(Y_{\theta}-Y_{B}\right)+Y_{B}=Y_{B}
$$




$$
\begin{aligned}
& \frac{x_{B}-x_{A}}{x^{\prime}{ }_{B}-x^{\prime}} \cdot\left(x_{B}-x_{B}\right)+\left(x^{\prime}{ }_{B}=x^{\prime}{ }_{B}\right. \\
& \frac{Y_{D}-Y_{C}}{Y^{\prime}{ }_{D}-Y^{\prime}{ }_{C}} \cdot\left(Y_{\psi}-Y_{C}\right)+\nabla_{C}^{\prime}=Y_{\psi}^{\prime} \\
& \frac{x_{D}-x_{C}}{x^{\prime}{ }_{D}-x_{C}^{\prime}} \cdot\left(x_{\psi}-x_{C}\right)+x_{C}^{\prime}=x_{\psi}^{\prime} \\
& \frac{x_{\theta}-x_{Y}}{X_{Q}^{\prime}-x_{\psi}^{\prime}} \cdot\left(x-x_{\psi}\right)+x_{\psi}=x_{\psi}^{\prime} \\
& \frac{Y_{\theta}-Y_{\psi}}{Y_{\theta}^{\prime}-Y_{\psi}} \cdot\left(Y-Y_{\psi}\right)+Y_{\psi^{\prime}}=Y_{P}^{\prime}
\end{aligned}
$$

6- Programa FORTRAN

Para se obter os resultados em maior velocidade e precisão, elaborou-se um programa ForTRAN, com as equações acima, cujo diagrama em blocos, programa e informações estão relacionados como se segue:

6.1- Diagrama em blocos 


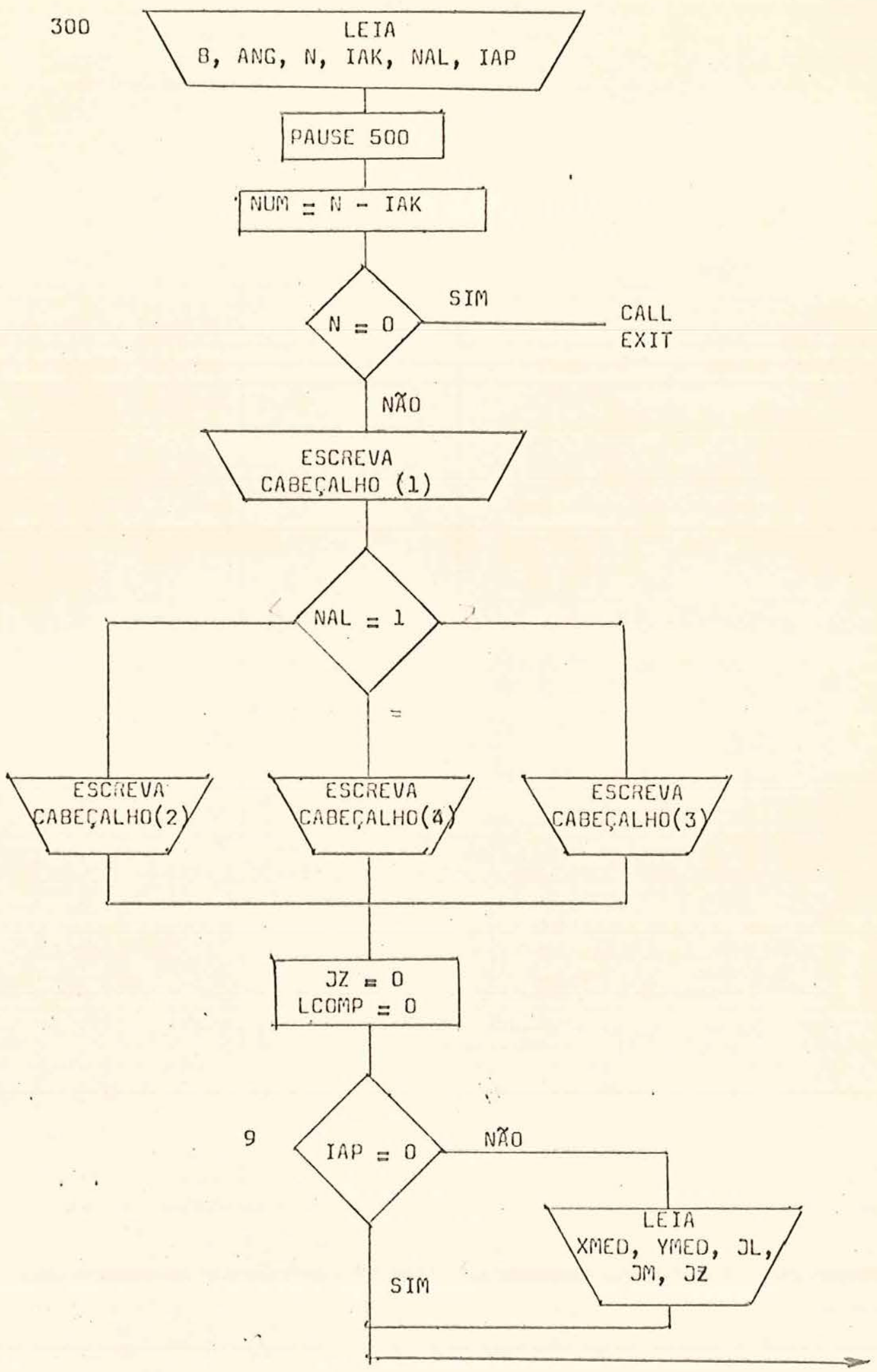




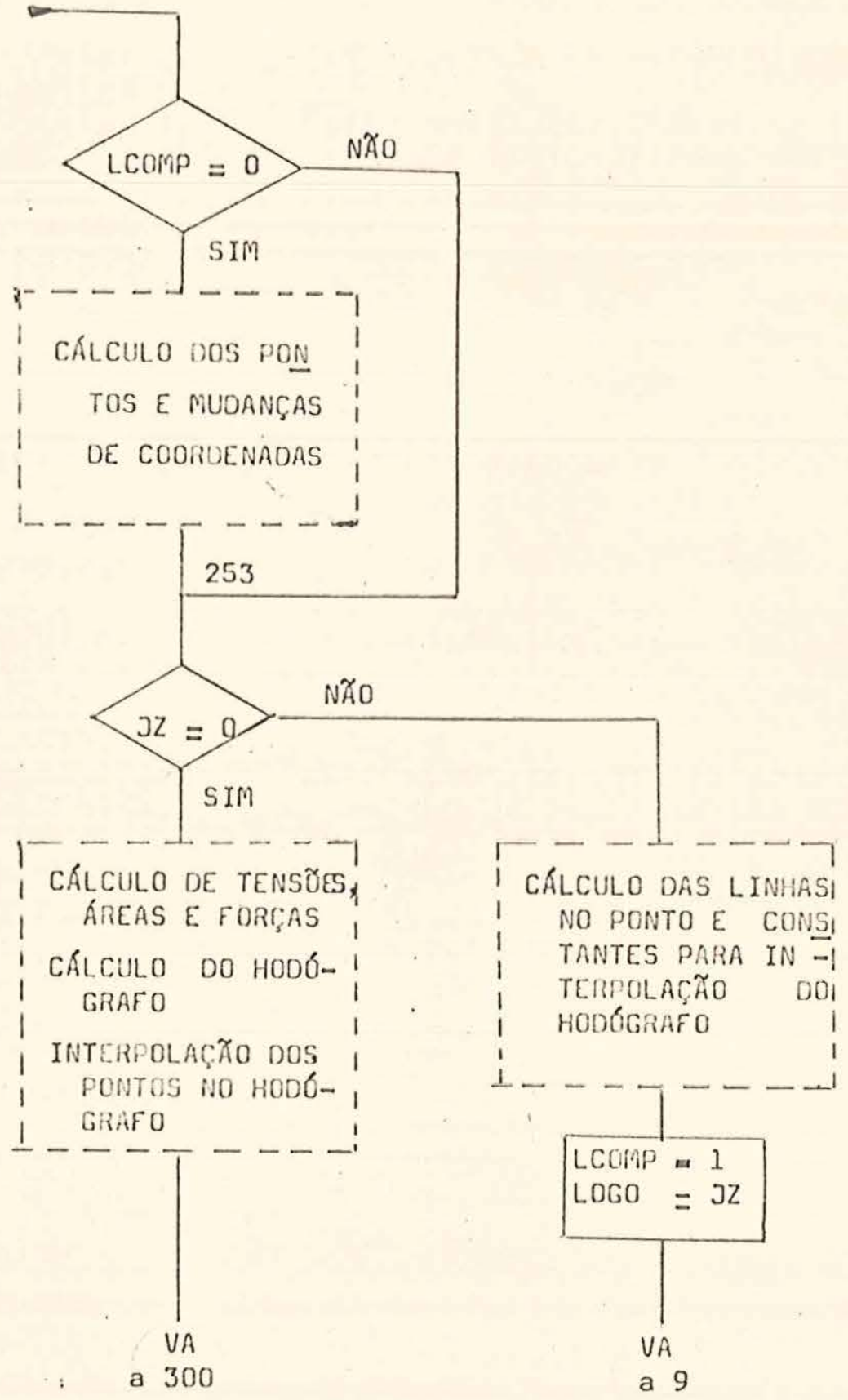

08 valores adiante não correspondem à fôrça de forjamento no exemplo dado, mas sim às fôrças devidas à uma línha de fluêncía. 
6.2- Listagem e resultados obtidos

PAGE 1 SAMUEL

// Jo8 i

SAMUEL $\quad 1$

$\begin{array}{cccc}\text { LOG DRIVE } & \text { CART SPEC } & \text { CART AVAIL } & \text { PHY DRIVE } \\ 0000 & 0016 & 0016 & 0000\end{array}$

V2 M1O ACTUAL $32 K$ CONFIG $32 K$

*EQUAT (PRNTZ, PRNZ)

// FOR

*IOCS (CARD, 11 32PRINTER, TYPEWR ITER, KEYBOARD, DISK,PLOTTER, ג403PRINTER)

*LIST SOURCE PROGRAM

DIMENSION X(18,18), Y $(18,18), A N(18,18), X G A M A(10), Y G A M A(10)$,

IXALFA $(10)$, YALFA $(10)$, XME (10), YME (10), RO1 (10), RO2(10),

$2 \mathrm{LOC}(10), \mathrm{MOC}(10), \mathrm{RO} 3(10), \mathrm{RO} 4(10)$

$300 \operatorname{READ}(2,1) B, A N G, N, I A K, N A L$, IAP

I FORMAT (2F6.3,4I2)

PAUSE 500

$N U M=N-I A K$

299 CONTINUE

WRITE $(3,69)$

69 FURMAT(10X,'ESTUDO DAS TENSOES PELO METODO DAS LINHAS DE FLUENCIA $.1, / /)$

IF (NAL-1)61,62,63

61 WRITE $(3,64) B, N$

64 FORMAT(IOX, 'OPERACAD FORJAMENTO COM MATRIZ FECHADA, //,

$110 X$, 'ALTURA DO FORJADO= $, F 6.3,1$ (CM) 1,8

$210 X,\left[2,{ }^{\circ}\right.$ LINHAS,$/ / / 1$

GO TO 298

62 WRITE $(3,65) 8$, ANG

65 FORMAT(10X, 'DPERACAO TREFILACAO',//,10X, 'LARGURA DA TREFILA', $1 F 6.3,7^{\prime}$ (CM) ',//,10X,'ANGULO DA TREFILA=',F6.3,' (GR)',///) $60 \quad 10298$

63 WRITE $(3,66) B$

66 FORMAT(LOX, 'OPERACAO FORJAMENTO COM MATRYZ ABERTA',//, 10X, I'LARGURA DA FERRAMENTA=',F6.3,' (CM)',///)

298 CONTINUE

$\mathrm{JZ}=0$

LCOMP $=0$

$9 \operatorname{IF}(\operatorname{IAP}) 6,7,6$

6 READ $(2,2) X M E D, Y M E D, J L, J M, J Z$

2 FORMAT $(2 F 6.3,312)$

7 CONTINUE

IF (LCOMP) $251,252,251$

251 GO TO 253

252 CONTINUE

$X A=0$.

$Y A=0$ 。

$X B=B$

$\mathrm{YB}=0$.

$B A N G=N N G / 5$.

GAH $=5$.

$C A B=3.1416 / 180$.

RAIO $=B /(2 \cdot * * 5)$

DO $20 \mathrm{~J}=1, \mathrm{~N}$

$\mathrm{C}=\mathrm{J}$

$B E=(45 .+(C-1) * G A M) * C A$.

$X(1, J)=B-R A I Q * \operatorname{CUS}(B E)$

$Y(1, J)=$ RAIO*SIN(BE) 
$20 Y(J, 1)=Y(1, J)$

$0030 \quad I=1, N$

$0030 \mathrm{~J}=1, \mathrm{~N}$

$\mathrm{C}, \mathrm{l}=\mathrm{l}$

$\mathrm{C} \cdot 2=\mathrm{J}$

BET $1=45 .+(C, 1-C 2) * C A M$

$B \in T 2=45 \circ+(C 2-C 1) * G A M$

$B E L 2=B E T 2$

$B F L 1=B E T 2$

$B E 1=B E T 1 * C A B$

$B E 2=B E T 2 * \mathrm{CAB}$

$\mathrm{L}=\mathrm{I}+\mathrm{I}$

$$
M=J \div 1
$$

$A C H 1=\operatorname{SIN}(B E 1) / \operatorname{COS}(B E 1)$

$A C H 2=\operatorname{SIN}(B E 2) / C O S(B E 2)$

$X(L, M)=(Y(I, M)-Y(L, J)+X(L, J) * A C H 1+X(I, M) * A C H 2) /(A C H 1+A C H 2)$

$Y(L, M)=(X(L, M)-X(L, J)) * A C H L+Y(L, J)$

30 AN $(I, J)=B E T I$

$\mathrm{YA}=\mathrm{Y}(\mathrm{N}, \mathrm{H})-\mathrm{YA}$

$X A=X A-X(N, N)$

$X B=X B-X(N, N)$

$Y B=Y(N, N)-Y B$

$0037 \mathrm{I}=1, \mathrm{~N}$

$0037 \mathrm{~J}=1, \mathrm{~N}$

$X(I, J)=X(Y, J)-X(N, N)$

$Y(I, J)=Y(N, N)-Y(I, J)$

37 CONT INUE:

$G A=A N G * C A B$

$\mathrm{CAS}=\operatorname{COS}(\mathrm{GA})$

$S U N=\operatorname{SIN}(G A)$

$K A=N-I A K$

DO $33 \mathrm{~J}=1, \mathrm{~N}$

DO $33 \mathrm{I}=1, \mathrm{KA}$

$\operatorname{AN}(I, J)=\operatorname{AN}(I, J)-A N G$

33 CONTINUE

$\operatorname{IF}(A N(K A, N)) 407,409,409$

407 DO $430 \quad \mathrm{~J}=1$, N

DO $430 \quad I=1, K A$

$\operatorname{AN}(I, J)=-\operatorname{AN}(I, J)$

430 CONTINUE:

409 CONTINUE:

$$
\begin{aligned}
& Y A=Y A-Y(K A, N) \\
& Y B=Y B-Y(K A, N) \\
& X A=X A-X(K A, N) \\
& X B=X B-X(K A, N) \\
& D O 34 J=1, N \\
& D O 34 I=1, K A \\
& X(I, J)=X(I, J)-X(K A, N)
\end{aligned}
$$

$34 Y(I, J)=Y(X, J)-Y(K A, N)$ $A X=X B$

$A Y=Y B$

$X B=A Y * S U N+A X * C A S$

$Y B=A Y * C A S-A X * S U N$

$A X=X A$

$A Y=Y A$

$X A=A Y * S U N+A X * C A S$

$Y A=A Y * C A S-A X * S U N$

WRITE $(3,412) \times A, Y A, X B, Y B$

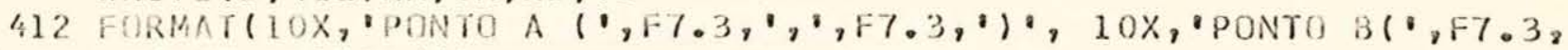
$10,1,(10,3,1) 0, / 1)$ 
PAGE 3 SAMULL.

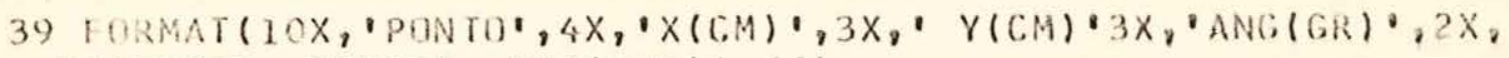
1'SEN2FI COS2FI $2 F I(R A D) \cdot, / /)$

$0035 \quad I=1, K A$

Dit $35 \mathrm{~J}=1, \mathrm{~N}$

$A X=X(I, J)$

$A Y=Y(I, J)$

$X([, J)=A Y * S U N+A X * C A S$

$Y(i, J)=A Y * C A S-A X * S U N$

$A L I Y=A N(I, J) * C A B$

$S A C=S I N(2, * A L F I)$

$C A C=\operatorname{COS}(2 \cdot * A L F I)$

$A L F 0=2 \cdot \therefore A L I I$

WRITE $(3,36) I, J, X(I, J), Y(I, J), A N(I, J), S A C, C A C, A L F O$

36 FORMAT $(10 X, 12, ', ', 12,3 X, F 7.3, F 7.3,2 X, F 7.3, F 8.4, F 8.4, F 11.4,1)$

35 CONTINU

IF(ANG -90.$) 440,431,431$

431 ANGU $=-1.57$

RAT $=-20$.

$\mathrm{CAD}=0$.

$\mathrm{RAD}=.5$

$B A D=1.5$

$A \cup D=0$.

Gu TO 437

440 CONTINUE

$$
\begin{aligned}
& \text { RAD }=0.0 \\
& C A D=.5 \\
& \text { BAD }=0 . \\
& \text { AUD }=1.5 \\
& \text { RAT }=0 . \\
& \text { ANGU }=0 .
\end{aligned}
$$

437 GONTINUE

CALL SCALF $(0.4,0.4,-60$, RAT $)$

$Z=X(1,1)$

$W=Y(1,1)$

CALL FPLOT $(-2, Z, W)$

CALL FPLOT $(0, X A, Y A)$

CALL. FPLOT $(0, X B, Y B)$

CALL FPLOT $(-2, Z, W)$

DO $800 \mathrm{~J}=1, \mathrm{~N}$

DO $301 \quad i=1, K A$

$\mathrm{Z}=\mathrm{X}(\mathrm{I}, \mathrm{J})$

$W=Y(I, J)$

801 CALL FPLOT $(-2, Z, W)$

$Z=X(K A, J)-A U D$

$W=Y(K A, J)+B A D$

CALL FCHAR $(Z, W, .10, .10$, ANGU)

800 WRITE $(7,802) \mathrm{J}$

802 FORHAT $(\cdot \mathrm{J}=1, \mathrm{I} 2)$

$Z=X(1,1)$

$W=Y(1,1)$

CALL FPLOT $(-2, Z, W)$

CALL POINT(I)

D0 $803 \quad I=1, K A$

DO $804 \mathrm{~J}=1, \mathrm{~N}$

$Z=X(I, J)$

$W=Y(I, J)$

804 CALL FPLOT $(-2, Z, W)$

$Z=X(I, N)+C A D$

$W=Y(I, N)-R A D$

CALL FCHAR $(L, W, .10, .10$, ANGU) 
PAGE 4 SAMU:L

805 FORMAT(' I=', I 2)

$X Y=X(K A, N)$

$Y I=Y(K A, N)$

CALL FPLOT $(2, X I, Y I)$

CALL FPLOT $(-3, X I, Y I)$

$Z O=X B \div .5$

CALL FPLOT $(2,20, Y I)$

$\angle Q=\angle D+.2$

CALL FPLOT $(-3, Z 0, Y T)$

CALL FPLOT $(2,20, Y I)$

CALL POINT(2)

$Z 0=Z O+.5$

WD $=Y I-.05$

CALL FCHAR $(Z 0, W 0, .10, .10,0$.

WRITE $(7,811)$

811 FORMAT $\left(X^{\prime}\right)$

CALL FPLOT $(-2, X i, Y i)$

$C E=Y A$

IF $(Y A) 461,462,462$

$461 \mathrm{CE}=-Y \mathrm{~A}$

462 CONTINUE

WO=CE+. 5

CALL FPLOT $(0, X I, W O)$

$W O=W O+.1$

CALL FPLOT $(2, X I, W O)$

CALL FPLOT $(-2, X Y$, WO $)$

CALL POINT(5)

$W O=W O+.5$

$Z 0=X I-.05$

CALL FCHAR $(Z 0, W O, .10, .10,0$.

WRITE $(7,809)$

809 FORMAT ( $\mathrm{Y}$ ')

$Z=X A+B A D$

$W=Y A+A U D$

CALL FCHAR(Z,W,.15,.20, ANGU)

WRITE $(7,1000)$

1000 FORMAT('LINHAS DE FLUENCIA')

CALL FPLOT $(0, X I, Y I)$

253 CONTINUE:

IF $(J Z) 3,4,3$

$3 X A O=X(J L, J M)$

$Y A D=Y(J L, J M)$

$J M 1=J M+1$

$X B Q=X(J L, J M I)$

$Y B O=Y(J L, J M I)$

$\mathrm{JLI}=\mathrm{JL}+1$

$X C O=X(j L 1, J M L)$

$\mathrm{YCO}=\mathrm{Y}(\mathrm{JL}, \mathrm{L}, \mathrm{JML})$

$X D D=X(J L 1, J M)$

$Y D Q=Y(J L 1, J M)$

CONS $I=(X B O * Y C O-X C O * Y B O) *(Y A O-Y D O)-(X A O * Y D O-X D O * Y A O) *(Y B O-Y C O)$

CONSO $=(Y B O-Y C O) *(X D O-X A O)-(X C O-X B O) *(Y A O-Y D O)$

$Y P O=$ CONS $1 /$ CONS2

$X P Q=(Y P O)(X D O-X A O)+X A O * Y D O-X D O * Y A O) /(Y D D-Y A O)$

CONS $)=(X P O * Y M E D-X M E D * Y P O) *(Y D O-Y C O)-(Y P D-Y M C D) *(X D O * Y C O-X C O * Y D O)$

CONSZ $=(X C O-X D O) \div(Y P D-Y M E D)-(Y D O-Y C O) *(X M E D-X P O)$

YALFA $(J /)=$ CONS1/CONS2

$X A L F A(J Z)=(Y A L F A(J Z) *(X M E O-X P O)+X P O * Y M E D-X M E D * Y P O) /(Y M E D-Y P O)$

CONS $1=(X A D * Y B D-X B O * Y A O) *(Y P O-Y M E D)-(X P O * Y M E D-X M E D * Y P O) *(Y A O-Y B O)$

COASZ $=(X M E D-X P D) *(Y A O-Y B O)-(X B O-X A O) *(Y P O-Y M E D)$ 
$X G A M A(J Z)=(Y G A M A(J Z) *(X B O-X A O)+X A O * Y B O-X B O * Y A O) /(Y B O-Y A D)$

$R O C L=(X U O-X C O) /(X A L F A(J Z)-X U O)$

$R O G 2=(Y D O-Y C O) /(Y A L F A(J Z)-Y D O)$

$J A=J M+1$

$X P T U=((X(1, J M)-X(1, J A)) / R O C 1)+X(1, J M)$

$Y P T O=((Y(1, J M)-Y(1, J A)) / R O C 2)+Y(1, J M)$

WRITE $(3,23) \mathrm{JL}, X M E D, Y M E D, J M, J A$

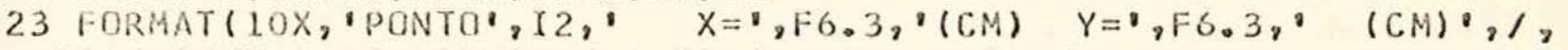

$110 X,{ }^{\circ}$ ENTRE AS LINHAS $\mathrm{J}=0,12,{ }^{\circ}$ E $\mathrm{J}=0,12,1$,

$\left.211 \times, \cdot 1,5 \times, \cdot \times(C M) \quad Y\left(C_{1}\right)^{\prime}, 1 / 1\right)$

$0021 \quad I=1, K A$

$X P T O=((X(I, J M)-X(I, J A)) / R O C I)+X(X, J M)$

$Y P T U=((Y(I, J M)-Y(I, J A)) / R O C 2)+Y(I, J M)$

ANGUL $=A N(I, J M)+30 C, 1 * G A M$

WRITE $(3,22) \mathcal{L}$, XPT, YPTO

22 FORMAT $(10 X, I 2,4 X, F 6.3,2 X, F 6.3,1)$

21 CONTINUE

$I A=J L+1$

WRITE $(3,24), \mathrm{L}, \mathrm{IA}$

24 FORMAT $(10 X$, LNTKE AS LINHAS $I=0, x 2, "$ E $I=0,12, / 1$,

$\left.111 \mathrm{X}, \mathrm{J}^{\prime}, 5 \mathrm{X}, \mathrm{X}(\mathrm{CM}) \quad \mathrm{Y}(\mathrm{CM}) \cdot, / 1\right)$

ROC $1=(X G A M A(J Z)-X A L F A(J Z)) /(X M E D-X G A M A(J Z))$

$\operatorname{RDC} 2=(Y G A M A(J Z)-Y A L F A(J Z)) /(Y M E D-Y G A M A(J Z))$

$X P T E=((X(J L, 1)-X(I A, I)) / R O C I)+X(J L, I)$

YPTO $=((Y(J L, 1)-Y(I A, 1)) / R O C 2)+Y(J L, I)$

DO $25 \mathrm{~J}=1, \mathrm{~N}$

$Y P T O=((Y(J L, J)-Y(I A, J)) / R O C 2)+Y(J L, J)$

$X P T U=((X(J L, J)-X(I A, J)) / R O C I)+X(J L, J)$

ANGUL $=A N(\mathrm{JL}, \mathrm{J})+R O C 1 * G A M$

WRITE $(3,26) \mathrm{J}$, XPTO, YPTO

26 FORMAT $(10 X, 12,4 X, F 6.3,2 X, F 6.3,1)$

25 CONTINUE

$X P T U=X M E D$

$\mathrm{YPTO}=\mathrm{YMEO}$

CALL FPLUT $(-2$, XPTO,YPTO)

CALL POINT(1)

$X M E(J Z)=X M E D$

$X M E D=X M E D+.1$

CALL FCHAR (XMED, YMED, $.10, .10,0$.

WRITE $(7,27) \mathrm{JZ}$

27 FORMAT (12)

ROI $(J Z)=(X B O-X A O) /(X G A M A(J Z)-X A O)$

$R O 2(J Z)=(Y B Q-Y A O) /(Y G A M A(J Z)-Y A O)$

$R 03(\mathrm{JZ})=(X C O-X D O) /(X A L F A(J Z)-X D O)$

$R 04(\mathrm{JZ})=(Y C O-Y D O) /(Y A L F A(J Z)-Y D O)$

YME $(J Z)=Y M E D$

$\operatorname{LOC}(J Z)=J L$

$\operatorname{MOC}(\mathrm{JZ})=J M$

LCOMP $=1$

$\operatorname{LOGO}=3 Z$

GO TO 9

4

CONTINUE

$X C=X I+20$.

CALL SCALF $(0.4,0.4, X C, 0$.

$S M=S \operatorname{SN}(A N(1,1) * C A B)$

$C 2=S M-2 \cdot * A N(1,1) * C A B$

$Y I B=Y B$

$Y Y A=Y A$

$X I A=X A$

$x I=-x b$ 
PAGE 6 SAMUEL

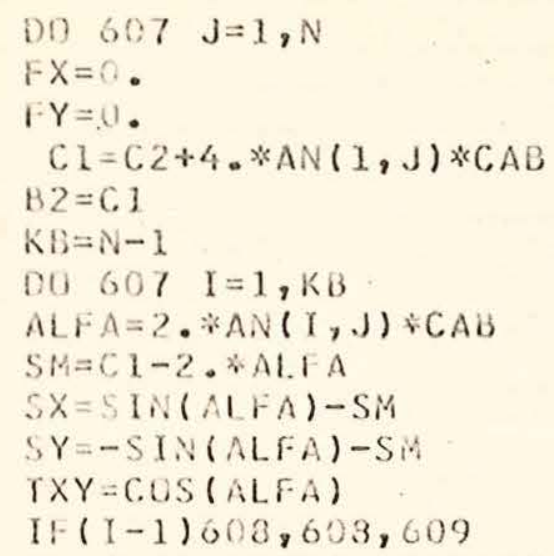

$608 A I=(Y(I, J) * 2 .-Y I B * 2) *$.

IF (A1) $509,509,510$

$509 \mathrm{Al}=-\mathrm{Al}$

$510 \quad A 2=(X(I, J)-X X B) * 6.2832$

IF (A2) $511,511,512$

$511 \quad A 2=-A 2$

$512 \quad F X=S X * A 1$

$F Y=S Y * A 2$

100 WRITE $(3,32) \mathrm{J}, \mathrm{BI}$

32 FORMAT(IOX,'PARA A LINHA $J=", 12, "$ COM C $1=1, F 7.3,1 /, 12 X^{\circ}$ PONTOS (TR
1 ( HOS)
AREA
SIGMA
FORCA
SOMA
TAU
$(0, / / 1$

WRITE $(3,400) A 1, S X, F X, F X, I, J, T X Y, B 2, A 2, S Y, F Y, F Y$

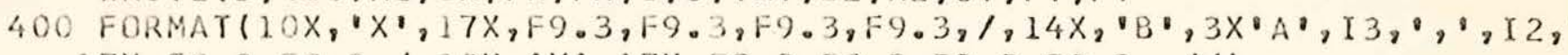

$17 X, F 9.3, F 9.3,1,10 X, 1 Y^{\circ}, 17 X, F 9.3, F 9.3, F 9.3, F 9.3,1 / 1$

$609 A I=(Y(I, J) * 2 .-Y(I+1, J) * 2) *$.

IF $(A)) 513,513,514$

$513 A 1=-41$

$514 A 2=(X(I, J)-X(X+1, J)) * 6.2832$

IF $(A 2) 515,515,516$

515 A.2 $=-A 2$

$516 \mathrm{Fl}=5 \mathrm{X} * \mathrm{Al}$

$F 2=S Y * A 2$

$F X=F 1+F X$

$F Y=F 2+F Y$

$J U M=I+1$

50 WRITE $(3,51) A 1, S X, F 1, F X, I, J, J U M, J, T X Y, B 2, A 2, S Y, F Z, F Y$

51 FORMATIIOX, 'X', I7X,F9.3,F9.3,F9.3,F9.3,/,12X, I2, ', ", I2, " A ', I2," $\left.1^{\prime}, I_{2}, 37 X, F 9.3, F 9.3, /, 10 X, Y^{\prime}, 17 X, F 9.3, F 9.3, F 9.3, F 9.3, / 1\right)$

607 CONTINUE

WRITE $(3,713)$

713 FORMAT(1OX, 'HODHGRAFO'//,

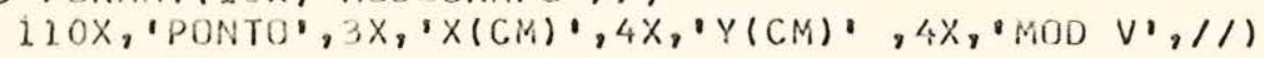

$X I A=-X A$

$X Y B=-X B$

IF $(A N G-90) 731,732,$.

731 CONTINUE

$A Y=Y, A$

$A X=X A$

$X A=A X * C A S-A Y * S U N$

$Y A=A X * S U N+A Y * C A S$

$A Y=Y B$

$A X=X B$

$Y B=A X * S U N+A Y * C A S$

$X B=A X * C A S-A Y * S U N$

$Y I A=Y A$

$X I A=-X A$

$Y I B=Y B$ 
IF (IAK) $977,978,977$

977 CONGINUE

$X O=-(X A+X B) / 2$.

60 ro 919

$978 X_{0}=-X_{A}-7$.

979 CONTINUE

$Y \mathrm{O}=(Y A+Y B) / 2$.

WRITE $(3,733)$ XIA, YIA, XIB, YIB

733 FURMAT(10X,' A ', $2 X, F 7.3,2 X, F 7.3,1$,

$110 x, 8 \quad, 2 X, F 7.3,2 X, F 7.3,11$

D0 $734 \quad I=1, K A$

DO $734 \mathrm{~J}=1, \mathrm{~N}$

$A Y=Y(I, J)$

$A X=X(I, J)$

$Y(X, J)=A X * S U N+A Y * C A S$

$X(I, J)=A X * C A S-A Y * S U N$

LUMA $=\mathrm{N} \div 1-\mathrm{J}$

$N U M=K A+1-1$

$X(I, J)=-X(I, J)$

DISTA $=\left((X 0-X(I, J)) * * 2_{0}+(Y O-Y(I, J)) * 2_{0}\right) * * x_{0}$

WRITE $(3,735)$ NUM, LUM, X $(I, J), Y(I, J)$, DISTA

735 FORMAT $\left(10 X, Y 2, x^{\prime}, Y 2,2 X, F 7.3,2 X, F 7.3,2 X, F 7.3,1\right)$

734 CONTINUE

60 TO 910

732. CONTINUE

WRITE $(3,714)$ XIA, YIA, XXB, YIB

714 FURMAT $(10 X$, A $, 2 X, F 7.3,2 X, F 7.3, \%$,

$110 X, \because B, 2 X, F 7.3,2 X, F 7.3,1)$

$X 0=-(X A+X B) / 2$.

$Y O=(Y A+Y B) / 2$.

DO $715 \quad I=1, K A$

DO $715 \mathrm{~J}=1, \mathrm{~N}$

$X(I, J)=-X(I, J)$

DISTA $=\left((X 0-X(I, J)) * * 2_{0}+(Y O-Y(I, J)) * * 2_{0}\right) * * .5$

NUM $=K A+1-I$

$L U M=N+1-J$

WRITE $(3,716)$ NUM, LUM, X $(I, J), Y(I, J), D I S T A$

716 FORMAT $\left(10 X_{2} 12,{ }^{\circ},, 12,2 X_{3} F 7.3,2 X_{2} F 7.3,2 X_{2} F 7.3,1\right)$

715 CONTTNUE

910 CONTINUE

$X A=-X A$

$X B=-X B$

$Z=X(1,1)$

$W=Y(1,1)$

CALL FPLOT $(-2, Z, W)$

CALL FPLOT $(0, X B, Y B)$

CALL FPLOT $(O, X A, Y A)$

CALL FPLOT $(0, Z, W)$

DO $900 \mathrm{~J}=1, \mathrm{~N}$

DO $901 \quad I=1, K A$

$Z=X(I, J)$

$W=Y(I, J)$

901 CALL FPLOT $(-2, Z, W)$

$Z=X(K A, J)+C A D$.

$W=Y(K A, J)+B A D$

LUM $=N \div 1-J$

CALL FCHAR $(Z, W, .10, .10$, ANGU)

900 WRITE $(7,902)$ LUM

902 FURMAT $(v \mathrm{~J}=1,12)$

$Z=X(1,1)$ 
CALL FPLET $(-2, \angle, W)$

$00903 \dot{I}=1, K A$

Do $904 \mathrm{~J}=1, \mathrm{~N}$

$z=X(1, j)$

$W=Y(I, j)$

904 CALL FPLOT $(-2, Z, W)$

$Z=X(I, N)-A U D$

$\mathrm{W}=Y(I, N)-R A D$

NUN $=K A+1-I$

CALL FCHAR $(Z, 6,010, .10, \operatorname{ANGU})$

903 WRITE $(7,905)$ NUM

905 FORMAT $(" 1=1,12)$

IF (ANG-90.) $871,872,872$

$371 \times I=X(K A, N)$

$Y I=Y(K A, N)$

$Z Y=X A$

$W Y=Y A$

GO TO 873

$872 \times I=(X A+X B) / 2$.

$Y I=(Y A+Y B) / 2$ 。

$Z I=X(K A, N)$

$W I=Y(K A, N)$

873 CONTINUE

CALL FPLOT $(2, X I, Y T)$

$Z U=Z T+.2$

CALL FPLOT $(-2, Z O, Y I)$

$\angle O=Z O H+.1$

CALL POINT(2)

CALL FPLOT $(2, Z 0, Y I)$

$Z O=Z O+.1$

$W Q=Y \Gamma-.05$

CALL FCHAR $(Z 0, W 0,010, .10,0$.

WRITE $(7,911)$

911 FORMAT(' $\left.x^{\prime}\right)$

CALL FPLOT $(-2, X 1, Y I)$

$W O=W I+2$.

CALL FPLOT $(0, X I, W O)$

CALL FPLOT $(2, X I, W O)$

$W 0=16 \div .1$

CALL FPLOT $(-2, X I, Y I)$

CALL POLATT 5 )

$W C=W C \div .1$

$Z[j=X i-.05$

CALL FCHAR $(20, W O, \cdot 10,010,0$.

WRT TE $(7,909)$

909 FORMAT('Y')

$Z=X A+B A D$

$W=Y A \div A U D$

CALL FCHAR ( $Z, W, .15, .20,-A N G U)$

WRITE $(7,1.001)$

1001 FORMAT('HODOGRAFO')

DO $46 \mathrm{JZ}=1, \mathrm{LOGO}$

$\mathrm{LUMO}=\mathrm{KA}+\mathrm{I}-\mathrm{LOC}(\mathrm{JZ})$

$N U M O=N+1-M O C(\mathrm{JZ})$

$N A M O=N U M O-1$.

$\mathrm{LAMO}=\mathrm{LU} H \mathrm{HO}-1$

$X G A M=((X(L A M O, N U M O)-X(L U M O, N U M O)) / R D 1(J Z))+X(L A M O, N U M O)$

$Y G A M=((Y(L A M O, N U M O)-Y(L U M O, N U M O)) / R O 2(J Z))+Y(L A M O, N U M O)$

$X / L F=((X(L A M D, N A M O)-X(L$ UMO, NAMO $)) / R O 3(\mathrm{JZ}))+X(L U M O, N A M O)$

$Y A H=((Y(L A M O, N A M O)-Y(L U M O$, KAMO $)) / R U 4(\mathrm{~J} \angle))+Y(L U M O, N A M O)$ 
PAGE 9 SAMULL

$Y P T=((Y G A M-Y A L F) /((Y G A M A(J Z)-Y A L F A(J Z)) /(Y M E(J Z)-X A L F A(J Z))))+Y A L$

CALL FPLOT $(-2, X P T, Y P T)$

CALL POINT (O)

$X T=X P T+.1$

CALL FCHAR(XT,YPT, .10,.10,0.)

WRITE $(7,44) \mathrm{JZ}$

44 FCRMAT (12)

46 CONIINUE

GO TO 300

301 CONTINU:

CALL EXIT

(EN)

UNREFERENCED STATEMENTS

100

50

FEATURES SUPPORTED

IUCS

CORE REQUTREMENTS FOR

COMMON O VARIABLES 2440 PROGRAM 4666

END GF COMPILATION

// XEQ

ESTUDO DAS TENSOES PELO METODO DAS LINHAS DE FLUENCYA

DPERACAO FORJAMENTO COM MATRIZ ABERTA

LARGURA DA FERRAMENTA $=7.000$ (CM)

6.2.1- Fontos da malha de linhas de fluência

PONTO A $(-3.525,21.913)$ PONTO B $(3.474,21.913)$

PONTO X(CM) Y(CM) ANG(GR) SEN2FI COS2FI $2 F I(R A D)$

$1,1 \quad-0.02518 .413 \quad 45.000 \quad 1.0000-0.0000 \quad 1.5707$

$\begin{array}{lllllll}1.2 & 0.292 & 13.121 & 40.000 & 0.9848 & 0.1736 & 1.3962\end{array}$

$\begin{array}{lllllll}1.3 & 0.635 & 17.858 & 35.000 & 0.9396 & 0.3420 & 1.2217\end{array}$

$\begin{array}{lllllll}1,4 & 0.999 & 17.626 & 30.000 & 0.8660 & 0.4999 & 1.0471\end{array}$

$\begin{array}{lllllll}1.5 & 1.382 & 17.427 & 25.000 & 0.7660 & 0.6427 & 0.8726\end{array}$

$\begin{array}{lllllll}1,6 & 1.781 & 17.262 & 20.000 & 0.6427 & 0.7660 & 0.6981\end{array}$

$\begin{array}{lllllll}1.7 & 2.193 & 17.132 & 15.000 & 0.5000 & 0.8660 & 0.5235\end{array}$

$\begin{array}{lllllll}1,8 & 2.614 & 17.038 & 10.000 & 0.3420 & 0.9396 & 0.3490\end{array}$

$\begin{array}{lllllll}1.9 & 3.042 & 16.982 & 5.000 & 0.1736 & 0.9848 & 0.1745\end{array}$

$\begin{array}{lllllll}1.10 & 3.474 & 16.963 & 0.000 & 0.0000 & 1.0000 & 0.0000\end{array}$ 


\begin{tabular}{|c|c|c|c|c|c|c|}
\hline 1,11 & 3.905 & 16.982 & -5.000 & -0.1736 & 0.9848 & -0.1745 \\
\hline 1,12 & 4.333 & 17.039 & -10.000 & -0.3420 & 0.9396 & -0.3490 \\
\hline 1,13 & 4.755 & 17.132 & -15.000 & -0.5000 & 0.8660 & -0.5235 \\
\hline 1,14 & 5.167 & 17.262 & -20.000 & -0.6427 & 0.7660 & -0.6981 \\
\hline 1,15 & 5.566 & 17.427 & -25.000 & -0.7660 & 0.6427 & -0.8726 \\
\hline 2,1 & -0.344 & 18.121 & 50.000 & 0.9848 & -0.1736 & 1.7453 \\
\hline 2,2 & -0.025 & 17.803 & 45.000 & 1.0000 & -0.0000 & 1.5707 \\
\hline 2,3 & 0.334 & 17.500 & 40.000 & 0.9848 & 0.1736 & 1.3962 \\
\hline 2,4 & 0.721 & 17.230 & 35.000 & 0.9396 & 0.3420 & 1.2217 \\
\hline 2,5 & 1.131 & 16.993 & 30.000 & 0.8660 & 0.4999 & 1.0471 \\
\hline 2,6 & 1.562 & 16.792 & 25.000 & 0.7660 & 0.6427 & 0.8726 \\
\hline 2,7 & 2.010 & 16.629 & 20.000 & 0.6427 & 0.7660 & 0.6981 \\
\hline 2,8 & 2.471 & 16.505 & 15.000 & 0.5000 & 0.8660 & 0.5235 \\
\hline 2,9 & 2.944 & 16.422 & 10.000 & 0.3420 & 0.9396 & 0.3490 \\
\hline 2,10 & 3.423 & 16.380 & 5.000 & 0.1736 & 0.9848 & 0.1745 \\
\hline 2,11 & 3.905 & 16.380 & 0.000 & 0.0000 & 1.0000 & 0.0000 \\
\hline 2,12 & 4.387 & 16.422 & -5.000 & -0.1736 & 0.9848 & -0.1745 \\
\hline 2,13 & 4.365 & 16.507 & -10.000 & -0.3420 & 0.9396 & -0.3490 \\
\hline 2,14 & 5.335 & 16.633 & -15.000 & -0.5000 & 0.8660 & -0.5235 \\
\hline 2,15 & 5.794 & 16.800 & -20.000 & -0.6427 & 0.7660 & -0.6981 \\
\hline 3,1 & -0.686 & 17.858 & 55.000 & 0.9396 & -0.3420 & 1.9198 \\
\hline 3,2 & -0.386 & 17.500 & 50.000 & 0.9848 & -0.1736 & 1.7453 \\
\hline 3,3 & -0.025 & 17.140 & 45.000 & 1.0000 & -0.0000 & 1.5707 \\
\hline 3,4 & 0.368 & 16.809 & 40.000 & 0.9848 & 0.1736 & 1.3962 \\
\hline 3,5 & 0.794 & 16.511 & 35.000 & 0.9396 & 0.3420 & 1.2217 \\
\hline 3,6 & 1.248 & 16.249 & 30.000 & 0.8660 & 0.4999 & 1.0471 \\
\hline 3,7 & 1.728 & 16.025 & 25.000 & 0.7660 & 0.6427 & 0.8726 \\
\hline 3,8 & 2.230 & 15.842 & 20.000 & 0.6427 & 0.7660 & 0.6981 \\
\hline 3,9 & 2.751 & 15.703 & 15.000 & 0.5000 & 0.8660 & 0.5235 \\
\hline 3,10 & 3.287 & 15.608 & 10.000 & 0.3420 & 0.9396 & 0.349 \\
\hline 3,11 & 3.834 & 15.560 & 5.000 & 0.1736 & 0.9848 & 0.1745 \\
\hline
\end{tabular}




\begin{tabular}{|c|c|c|c|c|c|c|}
\hline & & & & & & -46 \\
\hline 3,13 & $4 \cdot 944$ & 15.609 & -5.000 & -0.1736 & 0.9848 & -0.1745 \\
\hline 3,14 & 5.499 & 15.707 & -10.000 & -0.3420 & 0.9396 & -0.3490 \\
\hline 3,15 & 6.047 & 15.854 & -15.000 & -0.5000 & 0.8660 & -0.5235 \\
\hline 4,1 & -1.050 & 17.626 & 60.000 & 0.8660 & -0.5000 & 2.0943 \\
\hline 4,2 & -0.772 & 17.230 & 55.000 & 0.9396 & -0.3420 & 1.9198 \\
\hline 4,3 & -0.420 & 16.809 & 50.000 & 0.9848 & -0.1736 & 1.7453 \\
\hline 4,4 & -0.025 & 16.415 & 45.000 & 1.0000 & -0.0000 & 1.5707 \\
\hline 4,5 & 0.408 & 16.051 & 40.000 & 0.9848 & 0.1736 & 1.3962 \\
\hline 4,6 & 0.879 & 15.721 & 35.000 & 0.9396 & 0.3420 & 1.2217 \\
\hline 4,7 & 1. 384 & 15.429 & 30.000 & 0.8660 & 0.4999 & 1.0471 \\
\hline 4,3 & 1.921 & 15.179 & 25.000 & 0.7660 & 0.6427 & 0.8726 \\
\hline 4,9 & 2.485 & 14.973 & 20.000 & 0.6427 & 0.7660 & 0.6981 \\
\hline 4,10 & 3.074 & 14.815 & 15.000 & 0.5000 & 0.8660 & 0.5235 \\
\hline 4,11 & 3.683 & 14.708 & 10.000 & 0.3420 & 0.9396 & 0.3490 \\
\hline 4,12 & 4.308 & 14.653 & 5.000 & 0.1736 & 0.9848 & 0.1745 \\
\hline 4,13 & 4.944 & 14.653 & 0.000 & 0.0000 & 1.0000 & 0.0000 \\
\hline 4,14 & 5.586 & 14.710 & -5.000 & -0.1736 & 0.9848 & -0.1745 \\
\hline 4,15 & 6.229 & 14.823 & -10.000 & -0.3420 & 0.9396 & -0.3490 \\
\hline 5,1 & -1.433 & 17.427 & 65.000 & 0.7650 & -0.6427 & 2.2689 \\
\hline 5,2 & -1.183 & 16.993 & 60.000 & 0.8660 & -0.5000 & 2.0943 \\
\hline 5,3 & -0.345 & 16.511 & 55.000 & 0.9396 & -0.3420 & 1.9198 \\
\hline 5,4 & -0.459 & 16.051 & 50.000 & 0.9848 & -0.1736 & 1.7453 \\
\hline $5,5$. & -0.025 & 15.617 & 45.000 & 1.0000 & -0.0000 & 1.5707 \\
\hline 5,6 & 0.454 & 15.214 & 40.000 & 0.9848 & 0.1736 & 1.3962 \\
\hline 5,7 & 0.977 & 14.848 & 35.000 & 0.9396 & 0.3420 & 1.2217 \\
\hline 5,8 & 1.541 & 14.522 & 30.000 & 0.8660 & 0.4999 & 1.0471 \\
\hline 5,9 & 2.144 & 14.241 & 25.000 & 0.7660 & 0.6427 & 0.8726 \\
\hline 5,10 & 2.781 & 14.009 & 20.000 & 0.6427 & 0.7660 & 0.6981 \\
\hline 5,11 & 3.448 & 13.830 & 15.000 & 0.5000 & 0.8660 & 0.5235 \\
\hline 5,12 & 4.141 & 13.708 & 10.000 & 0.3420 & 0.9396 & 0.3490 \\
\hline 5,13 & 4.856 & 13.646 & 5.000 & 0.1736 & 0.9848 & 0.1745 \\
\hline
\end{tabular}




\begin{tabular}{|c|c|c|c|c|c|c|}
\hline 5,14 & 5.586 & 13.646 & 0.000 & 0.0000 & 1.0000 & 0.0000 \\
\hline 5,15 & 6.327 & 13.710 & -5.000 & -0.1736 & 0.9848 & -0.1745 \\
\hline 6,1 & -1.832 & 17.262 & 70.000 & 0.6427 & -0.7660 & 2.4434 \\
\hline 6,2 & -1.613 & 16.792 & 65.000 & 0.7660 & -0.6427 & 2.2689 \\
\hline 6,3 & -1.299 & 16.249 & 60.000 & 0.8660 & -0.5000 & 2.0943 \\
\hline 6,4 & -0.930 & 15.721 & 55.000 & 0.9396 & -0.3420 & 1.9198 \\
\hline 6,5 & -0.505 & 15.214 & 50.000 & 0.9848 & -0.1736 & 1.7453 \\
\hline 6,6 & -0.025 & 14.735 & 45.000 & 1.0000 & -0.0000 & 1.5707 \\
\hline 6,7 & 0.507 & 14.287 & 40.000 & 0.9848 & 0.1736 & 1.3962 \\
\hline 6,8 & 1.091 & 13.878 & 35.000 & 0.9396 & 0.3420 & 1.2217 \\
\hline 6,9 & 1.724 & 13.513 & 30.000 & 0.8060 & 0.4999 & 1.0471 \\
\hline 6,10 & 2.402 & 13.197 & 25.000 & 0.7660 & 0.6427 & 0.8726 \\
\hline 6,11 & 3.122 & 12.935 & 20.000 & 0.6427 & 0.7660 & 0.6981 \\
\hline 6,12 & 3.880 & 12.732 & 15.000 & 0.5000 & 0.8660 & 0.5235 \\
\hline 6,13 & 4.670 & 12.592 & 10.000 & 0.3420 & 0.9396 & 0.3490 \\
\hline 6,14 & 5.487 & 12.521 & 5.000 & 0.1736 & 0.9848 & 0.1745 \\
\hline 6,15 & 6.327 & 12.521 & 0.000 & 0.0000 & 1.0000 & 0.0000 \\
\hline 7,1 & -2.244 & 17.132 & 75.000 & 0.4999 & -0.8660 & 2.6179 \\
\hline 7,2 & -2.061 & 16.629 & 70.000 & 0.6427 & -0.7660 & 2.4434 \\
\hline 7,3 & -1.779 & 16.025 & 65.000 & 0.7660 & -0.6427 & 2.2689 \\
\hline 7,4 & -1.435 & 15.429 & 60.000 & 0.8660 & -0.5000 & 2.0943 \\
\hline 7,5 & -1.028 & 14.848 & 55.000 & 0.9396 & -0.3420 & 1.9198 \\
\hline 7,6 & -0.558 & 14.287 & 50.000 & 0.9848 & -0.1736 & 1.7453 \\
\hline 7,7 & -0.025 & 13.754 & 45.000 & 1.0000 & -0.0000 & 1.5707 \\
\hline 7,8 & 0.568 & 13.256 & 40.000 & 0.9848 & 0.1736 & 1.3962 \\
\hline 7,9 & 1.223 & 12.797 & 35.000 & 0.9396 & 0.3420 & 1.2217 \\
\hline 7,10 & 1.934 & 12.387 & 30.000 & 0.8660 & 0.4999 & 1.0471 \\
\hline 7,11 & 2.700 & 12.030 & 25.000 & 0.7660 & 0.6427 & 0.8726 \\
\hline 7,12 & 3.516 & 11.733 & 20.000 & 0.6427 & 0.7560 & 0.6981 \\
\hline 7,13 & 4.378 & 11.502 & 15.000 & 0.5000 & 0.8660 & 0.5235 \\
\hline 7,14 & 5.280 & 11.343 & 10.000 & 0.3420 & 0.9396 & 0.3490 \\
\hline
\end{tabular}




\begin{tabular}{|c|c|c|c|c|c|c|}
\hline 8,1 & -2.666 & 17.038 & 80.000 & 0.3420 & -0.9396 & 2.7925 \\
\hline 8,2 & -2.523 & 16.505 & 75.000 & 0.4999 & -0.8660 & 2.6179 \\
\hline 8,3 & -2.281 & 15.842 & 70.000 & 0.6427 & -0.7660 & 2.4434 \\
\hline 8,4 & -1.972 & 15.179 & 65.000 & 0.7660 & -0.6427 & 2.2689 \\
\hline 3,5 & -1.593 & 14.522 & 60.000 & 0.8660 & -0.5000 & 2.0943 \\
\hline 8,6 & -1.142 & 13.378 & 55.000 & 0.9396 & -0.3420 & 1.9198 \\
\hline 3,7 & -0.620 & 13.256 & 50.000 & 0.9848 & -0.1736 & 1.7453 \\
\hline 8,8 & -0.025 & 12.661 & 45.000 & 1.0000 & -0.0000 & 1.5707 \\
\hline 8,9 & 0.640 & 12.102 & 40.000 & 0.9848 & 0.1736 & 1.3962 \\
\hline 8,10 & 1.375 & 11.588 & 35.000 & 0.9396 & 0.3420 & 1.2217 \\
\hline 8,11 & 2.177 & 11.124 & 30.000 & 0.8660 & 0.4999 & 1.0471 \\
\hline 8,12 & 3.64 & 10.720 & 25.000 & 0.7660 & 0.6427 & 0.8726 \\
\hline 8,13 & 3.970 & 10.383 & 20.000 & 0.6427 & 0.7660 & 0.6981 \\
\hline 8,14 & $4 \cdot 52$ & 10.120 & 15.000 & 0.5000 & 0.8660 & 0.5235 \\
\hline 8.25 & 5.983 & 9.938 & 10.000 & 0.3420 & 0.9396 & 0.3490 \\
\hline 9,1 & -3.094 & 16.982 & 85.000 & 0.1736 & -0.9848 & 2.9670 \\
\hline 9,2 & -2.395 & 16.422 & 80.000 & 0.3420 & -0.9396 & 2.7925 \\
\hline 9,3 & -2.802 & 15.703 & 75.000 & 0.4999 & -0.8660 & 2.6179 \\
\hline 9,4 & -2.537 & 14.973 & 70.000 & 0.6427 & -0.7660 & 2.4434 \\
\hline 9,5 & -2.195 & 14.241 & 65.000 & 0.7660 & -0.6427 & 2.2689 \\
\hline 9,6 & -1.775 & 13.513 & 60.000 & 0.8660 & -0.5000 & 2.0943 \\
\hline 9,7 & -1.274 & 12.797 & 55.000 & 0.9396 & -0.3420 & 1.9198 \\
\hline 9,8 & -0.691 & 12.103 & 50.000 & 0.9848 & -0.1736 & 1.7453 \\
\hline 9,9 & -0.025 & 11.437 & 45.000 & 1.0000 & -0.0000 & 1.5707 \\
\hline 9,10 & 0.722 & 10.809 & 40.000 & 0.9848 & 0.1736 & 1.3962 \\
\hline 9,11 & 1.550 & 10.229 & 35.000 & 0.9396 & 0.3420 & 1.2217 \\
\hline 9,12 & 2.458 & 9.705 & 30.000 & 0.8660 & 0.4999 & 1.0471 \\
\hline 9,13 & 3.441 & 9.247 & 25.000 & 0.7660 & 0.6427 & 0.8726 \\
\hline 9.14 & 4.495 & 3.863 & 20.000 & 0.6427 & 0.7660 & 0.6981 \\
\hline 9,15 & 5.615 & 8.563 & 15.000 & 0.5000 & 0.8660 & 0.5235 \\
\hline & 525 & 16. & 90.000 & -0.0000 & -1 & 3. \\
\hline
\end{tabular}




\begin{tabular}{|c|c|c|c|c|c|c|}
\hline 10,2 & -3.474 & 16.380 & 85.000 & 0.1736 & -0.9848 & 2.9670 \\
\hline 10,3 & -3.338 & 15.608 & 80.000 & 0.3420 & -0.9396 & 2.7925 \\
\hline 10,4 & -3.126 & 14.815 & 75.000 & 0.4999 & -0.8660 & 2.6179 \\
\hline 10,5 & -2.832 & 14.009 & 70.000 & 0.6427 & -0.7660 & 2.4434 \\
\hline 0,6 & -2.453 & 13.197 & 65.000 & 0.7660 & -0.6427 & 2.2689 \\
\hline 10,7 & -1.936 & 12.387 & 60.000 & 0.8660 & -0.5000 & 2.0943 \\
\hline 10,8 & -1.426 & 11.588 & 55.000 & 0.9396 & -0.3420 & 1.9198 \\
\hline 10,9 & -0.773 & 10.809 & 50.000 & 0.9848 & -0.1736 & 1.7453 \\
\hline 10,10, & -0.025 & 10.061 & 45.000 & 1.0000 & -0.0000 & 1.5707 \\
\hline 10,11 & 0.817 & 9.354 & 40.000 & 0.9848 & 0.1736 & 1.3962 \\
\hline 10,12 & 1.753 & 8.699 & 35.000 & 0.9396 & 0.3420 & 1.2217 \\
\hline 10,13 & 2.781 & 8.105 & 30.000 & 0.8660 & 0.4999 & 1.0471 \\
\hline 10,14 & 3.898 & 7.584 & 25.000 & 0.7660 & 0.6427 & 0.8726 \\
\hline 10,15 & 5.099 & 7.147 & 20.000 & 0.6427 & 0.7660 & 0.6981 \\
\hline 11,1 & -3.357 & 16.932 & 95.000 & -0.1736 & -0.9848 & 3.3161 \\
\hline 11,2 & -3.957 & 16.394 & 90.000 & -0.0000 & -1.0000 & 3.1415 \\
\hline 11,3 & -3.884 & 15.560 & 85.000 & 0.1736 & -0.9848 & 2.9670 \\
\hline 11,4 & -3.733 & 14.708 & 80.000 & 0.3420 & -0.9396 & 2.7925 \\
\hline 11,5 & -3.498 & 13.831 & 75.000 & 0.4999 & -0.8660 & 2.6179 \\
\hline 11,6 & -3.172 & 12.935 & 70.000 & 0.6427 & -0.7660 & 2.4434 \\
\hline 11,7 & -2.750 & 12.030 & 65.000 & 0.7660 & -0.6427 & 2.2689 \\
\hline 11,8 & -2.228 & 11.125 & 60.000 & 0.8660 & -0.5000 & 2.0943 \\
\hline 11,9 & -1.601 & 10.230 & 55.000 & 0.9396 & -0.3420 & 1.9198 \\
\hline 11,10 & -0.867 & 9.355 & 50.000 & 0.9848 & -0.1736 & 1.7453 \\
\hline 11,12 & -0.024 & 8.512 & 45.000 & 1.0000 & -0.0000 & 1.5707 \\
\hline 11,12 & 0.927 & 7.71 .4 & 40.000 & 0.9848 & 0.1736 & 1.3962 \\
\hline 11,13 & 1.987 & 6.971 & 35.000 & 0.9396 & 0.3420 & 1.2217 \\
\hline $1.1,14$ & 3.155 & 6.297 & 30.000 & 0.8660 & 0.4999 & 1.0471 \\
\hline & 4.426 & 5.704 & 25.000 & 0.7660 & 0.6427 & 0.8726 \\
\hline
\end{tabular}




\begin{tabular}{|c|c|c|c|c|c|c|}
\hline 12,1 & -4.385 & 17.039 & 100.000 & -0.3420 & -0.9396 & 3.4906 \\
\hline 12,2 & -4.437 & 16.436 & 95.000 & -0.1736 & -0.9848 & 3.3161 \\
\hline 12,3 & -4.437 & 15.624 & 90.000 & -0.0000 & -1.0000 & 3.1415 \\
\hline 12,4 & $-4 \cdot 353$ & 14.654 & 85.000 & 0.1736 & -0.9848 & 2.9670 \\
\hline 12,5 & $-4 \cdot 186$ & 13.709 & 80.000 & 0.3420 & -0.9396 & 2.7925 \\
\hline 12,6 & -3.925 & 12.734 & 75.000 & 0.4999 & -0.8660 & 2.6179 \\
\hline 12,7 & -3.561 & 11.735 & 70.000 & 0.6427 & -0.7660 & 2.4434 \\
\hline 12,8 & -3.089 & 10.723 & 65.000 & 0.7660 & -0.6427 & 2.2689 \\
\hline 12,9 & -2.503 & 9.709 & 60.000 & 0.8660 & -0.5000 & 2.0943 \\
\hline 12,10 & -1.799 & 8.702 & 55.000 & 0.9396 & -0.3420 & 1.9198 \\
\hline 12,11 & -0.972 & 7.717 & 50.000 & 0.9848 & -0.1736 & 1.7453 \\
\hline 12,12 & -0.021 & 6.765 & 45.000 & 1.0000 & -0.0000 & 1.5707 \\
\hline 12,13 & 1.056 & 5.861 & 40.000 & 0.9848 & 0.1736 & 1.3962 \\
\hline 12,14 & 2.260 & 5.018 & 35.000 & 0.9396 & 0.3420 & 1.2217 \\
\hline 12,15 & 3.588 & 4.252 & 30.000 & 0.8660 & 0.4999 & 1.0471 \\
\hline 13,1 & -4.806 & 17.132 & 105.000 & -0.5000 & -0.8660 & 3.6651 \\
\hline 13,2 & -4.914 & 16.520 & 100.000 & -0.3420 & -0.9396 & 3.4906 \\
\hline 13,3 & -4.988 & 15.672 & 95.000 & -0.1736 & -0.9848 & 3.3161 \\
\hline 13,4 & $-4 \cdot 988$ & 14.698 & 90.000 & -0.0000 & -1.0000 & 3.1415 \\
\hline 13,5 & -4.896 & 13.647 & 85.000 & 0.1736 & -0.9848 & 2.9670 \\
\hline 13,6 & -4.711 & 12.595 & 80.000 & 0.3420 & -0.9396 & 2.7925 \\
\hline 13,7 & -4.419 & 11.505 & 75.000 & 0.4999 & -0.8660 & 2.6179 \\
\hline 13,8 & -4.012 & 10.387 & 70.000 & 0.6427 & -0.7660 & 2.4434 \\
\hline 13,$9 ;$ & -3.483 & 9.252 & 65.000 & 0.7660 & -0.6427 & 2.2689 \\
\hline 13,10 & -2.824 & 8.111 & 60.000 & 0.8660 & -0.5000 & 2.0943 \\
\hline 13,11 & -2.030 & 6.977 & 55.000 & 0.9396 & -0.3420 & 1.9198 \\
\hline 13,12 & -1.096 & 5.863 & 50.000 & 0.9848 & -0.1736 & 1.7453 \\
\hline 13,13 & -0.018 & 4.786 & 45.000 & 1.0000 & -0.0000 & 1.5707 \\
\hline 13,14 & 1.204 & 3.760 & 40.000 & 0.9848 & 0.1736 & 1.3962 \\
\hline 13,15 & 2.573 & 2.801 & 35.000 & 0.9396 & 0.3420 & 1.2217 \\
\hline 14,1 & -5.218 & 17.262 & 110.000 & -0.6427 & -0.7660 & 3.8397 \\
\hline 4,2 & -5.383 & 16.646 & 105.000 & -0.5000 & -0.3660 & 3.6651 \\
\hline
\end{tabular}


$14,3 \quad-5.53815 .769 \quad 100.000-0.3420-0.9396$

3.4906

$14.4 \quad-5.627 \quad 14.754 \quad 95.000-0.1736-0.9848$

3.3161

$14,5 \quad-5.62713 .780 \quad 90.000-0.0000-1.0000$

3.14 .15

$14,6 \quad-5.517 \quad 12.524 \quad 85.000 \quad 0.1736-0.9848$

2.9670

14,7

$-5.30911 .348$

$30.000 \quad 0.3420-0.9396$

2.7925

$14,8 \quad-4.982 \quad 10.127$

$75.000 \quad 0.4999-0.8660$

2.6179

$14,9 \quad-4.525 \quad 8.872 \quad 70.000 \quad 0.6427-0.7660$

2.4434

14,10

$-3.930 \quad 7.595$

65.000

$0.7660-0.6427$

2.2689

14,11

$-3.187 \quad 6.308$

60.000

$0.8660-0.5000^{\circ}$

2.0943

14,12

$-2.290 \quad 5.027$

55.000

$0.9396-0.3420$

1.9198

14,13

$-1.232 \quad 3.767$

50.000

$0.9848-0.1736$

1.7453

14,14

$-0.010 \quad 2.545$

45.000

$1.0000-0.0000$

1.5707

14,15

$1.379 \quad 1.379$

40.000

0.9848

0.1736

1.3962

15, 1

$-5.617 \quad 17.427$

$115.000-0.7660-0.6427$

4.0142

15,2

$-5.841 \quad 16.812$

$110.000-0.6427-0.7660$

3.8397

15,3

$-6.08115 .915$

$105.000-0.5000-0.8660$

3.6651

15,4

$-6.26614 .867$

$100.000-0.3420-0.9396$

3.4906

15,5

$-6.356 \quad 13.844$

$95.000-0.1736-0.9848$

3.3161

15,6

$-6.035612 .708$

$90.000-0.0000-1.0000$

3.1415

15,7

$-6.23011 .268$

85.000

$0.1736-0.9848$

2.9670

15,8

$-5.9979 .948$

80.000

$0.3420-0.9396$

2.7925

15,

$-5.629 \quad 8.577$

75.000

$0.4999-0.8660$

2.6179

15.10

$-5.1157 .164$

70.000

$0.6427-0.7660$

2.4434

15,11

$-4.443 \quad 5.723$

65.000

$0.7660-0.6427$

2.2689

15,12

$-3.604 \quad 4.269$

60.000

$0.8660-0.5000$

2.0943

15,13

$\begin{array}{ll}-2.588 & 2.818\end{array}$

55.000

$0.9396-0.3420$

1.9198

15,14

$-1.388 \quad 1.388$

50.000

$0.9848-0.1736$

1.7453

15,15

$0.000 \quad 0.000$

45.000

$1.0000-0.0000$

1.5707

6.2.2- pontos interpolados

ENTRE AS LINHAS $J=14$ I

$X(C M) \quad Y(C M)$

$Y=5.200$

E $J=1.5$

(CM) 


$\begin{array}{lll}2 & 5.622 & 16.737 \\ 3 & 5.841 & 15.799 \\ 4 & 5.988 & 14.780 \\ 5 & 6.048 & 13.686 \\ 6 & 6.011 & 12.521 \\ 7 & 5.865 & 11.291 \\ 8 & 5.596 & 10.006 \\ 9 & 5.194 & 8.676 \\ 10 & 4.648 & 7.311 \\ 11 & 3.949 & 5.927 \\ 12 & 3.089 & 4.540 \\ 13 & 2.058 & 3.161 \\ 14 & 0.357 & 1.817 \\ 15 & -0.521 & 0.521\end{array}$

ENTRE AS LINHAS $I=11$

E $\quad I=12$

$J \quad X(C M) \quad Y(C M)$

$1 \quad-4.181 \quad 17.012$

$2 \quad-4.208 \quad 16.416$

$3 \quad-4.174 \quad 15.594$

$4 \quad-4.058 \cdot 14.680$

$5 \quad-3.859 \quad 13.767$

6. $\quad-3.566 \quad 12.829$

$\begin{array}{lll}7 & -3.175 & 11.875\end{array}$

$8 \quad-2.679 \quad 10.914$

$9 \quad-2.074 \quad 9.957$

$10 \quad-1.355 \quad 9.013$

$11 \quad-0.521 \quad 8.096$

$12 \quad 0.430 \quad 7.217$

$131.500 \quad 6.390$

$14 \quad 2.686 \quad 5.627$ 
PONTO $2 \quad X=3.500(\mathrm{CM}) \quad Y=7.400 \quad$ (CM)

ENTRE AS LINHAS $J=13 \quad$ E $J=14$

I $\quad X(C M) \quad Y(C M)$

$\begin{array}{lll}1 & 5.083 & 17.236 \\ 2 & 5.240 & 16.607 \\ 3 & 5.386 & 15.687 \\ 4 & 5.456 & 14.698 \\ 5 & 5.438 & 13.646 \\ 6 & 5.322 & 12.535 \\ 7 & 5.097 & 11.375 \\ 8 & 4.753 & 10.173 \\ 9 & 4.231 & 8.941 \\ 10 & 3.672 & 7.689 \\ 11 & 2.919 & 6.433 \\ 12 & 2.016 & 5.189 \\ 13 & 0.956 & 3.968 \\ 14 & -0.258 & 2.792 \\ 15 & -1.631 & 1.678\end{array}$

ENTRE AS LINHAS $I=10 \quad$ E $I=11$

$J \quad X(C M) \quad Y(C M)$

$1 \quad-3.625 \quad 16.968$

$2 \quad-3.585 \quad 16.383$

$3 \quad-3.464 \quad 15.597$

$4 \quad-3.266 \quad 14.791$

$5 \quad-2.986 \quad 13.968$

$6 \quad-2.619 \quad 13.136$

$7 \quad-2.162 \quad 12.304$

$8 \quad-1.611 \quad 11.481$

$9 \quad-0.964 \quad 10.676$

$10 \quad-0.219 \quad 9.899$

$110.623 \quad 9.160$ 


$\begin{array}{lll}12 & 1.563 & 8.472 \\ 13 & 2.598 & 7.844 \\ 14 & 3.727 & 7.287 \\ 15 & 4.944 & 6.814\end{array}$

PONTO $3 \quad X=1.500(\mathrm{CM}) \quad Y=8.000 \quad$ (CM) CNTRE AS LINGAS $J=12$
$X(C M) \quad Y(C M)$$\quad$ E $J=13$

$\begin{array}{lll}1 & 4.420 & 17.058 \\ 2 & 4.486 & 16.440 \\ 3 & 4.502 & 15.570 \\ 4 & 4.439 & 14.653 \\ 5 & 4.288 & 1.3 .695 \\ 6 & 4.042 & 12.703 \\ 7 & 3.693 & 11.685 \\ 8 & 3.234 & 10.651 \\ 9 & 2.660 & 9.611 \\ 10 & 1.964 & 8.576 \\ 11 & 1.145 & 7.561 \\ 12 & 0.200 & 6.580 \\ 13 & -0.874 & 5.642 \\ 14 & -2.072 & 4.768 \\ 15 & -3.395 & 3.970\end{array}$

ENTRE AS LINMAS $I=10 \quad$ E $Y=11$

$J \quad X(C M) \quad Y(C M)$

$1 \quad-3.770 \quad 16.974$

$2:-3.748 \quad 16.388$

$3-3.648 \quad 15.581$

$4 \quad-3.471 \quad 14.755$

$5 \quad-3.210 \quad 13.908$

$6 \quad-2.862 \quad 13.048$

$7 \quad-2.420 \quad 12.184$ 


$\begin{array}{rrr}9 & -1.243 & 10.480 \\ 10 & -0.503 & 9.660 \\ 11 & 0.338 & 8.876 \\ 12 & 1.284 & 8.139 \\ 13 & 2.330 & 7.461 \\ 14 & 3.476 & 6.853 \\ 15 & 4.717 & 6.378\end{array}$

6.2.3- Tensões, áreas e forcas nos trechos

PARA A LINHA $J=1$ COM C $L=-0.863$

\begin{tabular}{|c|c|c|c|c|c|c|c|c|}
\hline PONTO: & SIT & RECHOS) & AREA & SIGMA & FORCA & SOMA & TAU & $\mathrm{C}$ \\
\hline$B$ & A & 1,1 & $\begin{array}{r}443.420 \\
21.991\end{array}$ & $\begin{array}{r}1.863 \\
-0.136\end{array}$ & $\begin{array}{r}826.399 \\
-2.997\end{array}$ & $\begin{array}{r}826.399 \\
-2.997\end{array}$ & -0.000 & 2.277 \\
\hline 1,1 & A & 2,1 & $\begin{array}{r}33.483 \\
2.000\end{array}$ & $\begin{array}{r}1.863 \\
-0.136\end{array}$ & $\begin{array}{r}62.403 \\
-0.272\end{array}$ & $\begin{array}{r}888.803 \\
-3.270\end{array}$ & -0.000 & 2.277 \\
\hline 2,1 & A & 3,1 & $\begin{array}{r}29.713 \\
2.152\end{array}$ & $\begin{array}{l}2.197 \\
0.227\end{array}$ & $\begin{array}{r}65.297 \\
0.490\end{array}$ & $\begin{array}{r}954.101 \\
-2.779\end{array}$ & -0.173 & 2.277 \\
\hline 3,1 & $A$ & 4,1 & $\begin{array}{r}25.865 \\
2.288\end{array}$ & $\begin{array}{l}2.501 \\
0.622\end{array}$ & $\begin{array}{r}64.702 \\
1.423\end{array}$ & $\begin{array}{r}1018.803 \\
-1.355\end{array}$ & -0.342 & 2.277 \\
\hline 4.1 & A & 5,1 & $\begin{array}{r}21.957 \\
2.406\end{array}$ & $\begin{array}{l}2.776 \\
1.044\end{array}$ & $\begin{array}{c}60.974 \\
2.514\end{array}$ & $\begin{array}{r}1079.778 \\
1.158\end{array}$ & -0.500 & 2.277 \\
\hline 5,1 & A & 6,1 & $\begin{array}{r}28.008 \\
2.506\end{array}$ & $\begin{array}{l}3.025 \\
1.493\end{array}$ & $\begin{array}{r}54.494 \\
3.744\end{array}$ & $\begin{array}{r}1134.272 \\
4.903\end{array}$ & -0.642 & 2.277 \\
\hline 6,1 & A & 7,1 & $\begin{array}{r}14.030 \\
2.587\end{array}$ & $\begin{array}{l}3.251 \\
1.966\end{array}$ & $\begin{array}{r}45.624 \\
5.087\end{array}$ & $\begin{array}{r}1179.897 \\
9.991\end{array}$ & -0.766 & 2.277 \\
\hline 7,1 & A & 8,1 & $\begin{array}{r}10.033 \\
2.648\end{array}$ & $\begin{array}{l}3.458 \\
2.458\end{array}$ & $\begin{array}{r}34.695 \\
6.511\end{array}$ & $\begin{array}{r}1214.592 \\
16.502\end{array}$ & -0.866 & 2.277 \\
\hline 8,1 & $A$ & 971 & 6.023 & 3.649 & 21.980 & 1236.573 & -0.939 & 2.27 \\
\hline
\end{tabular}




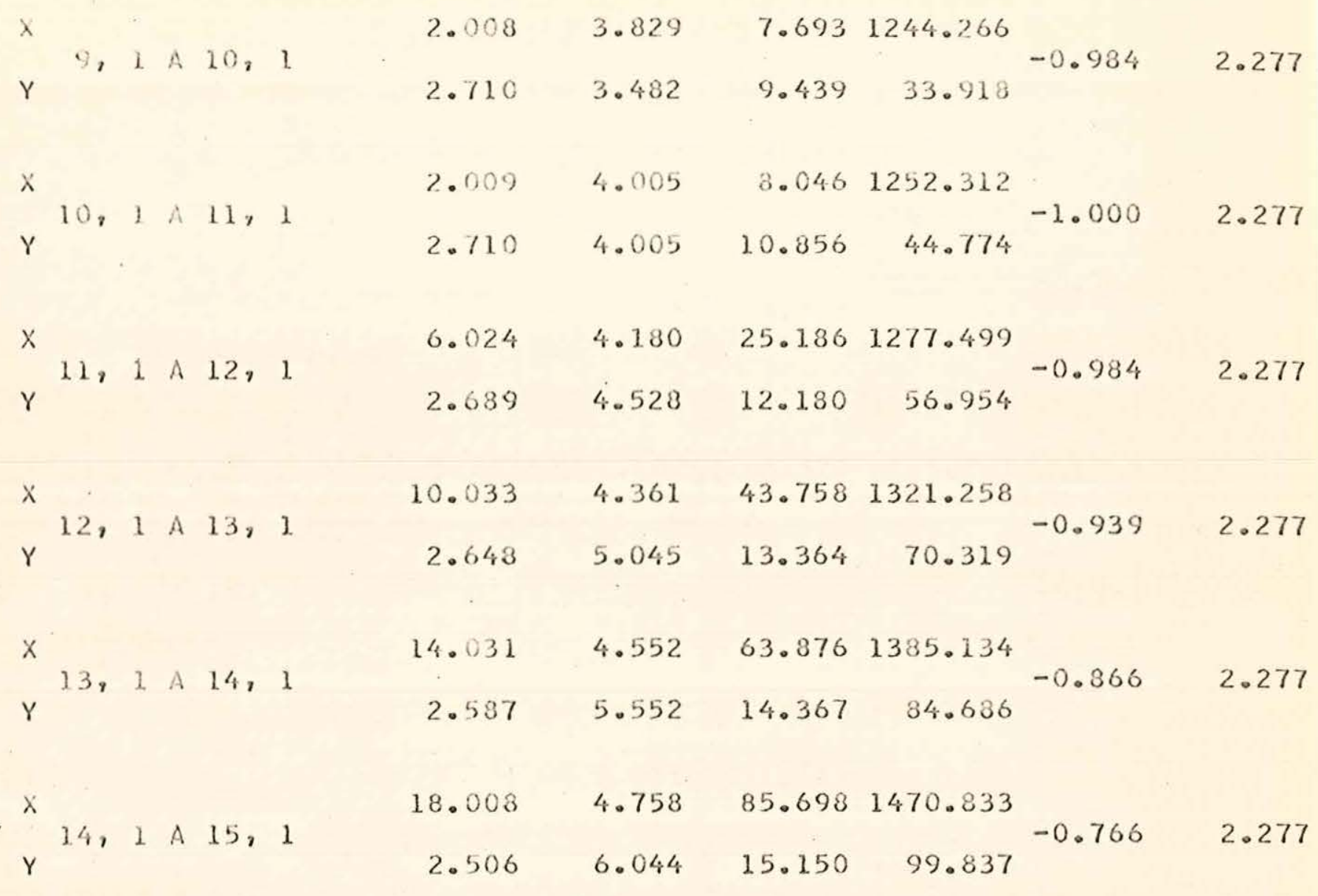

PARA A LINHA $J=2 \operatorname{COM} C I=-0.863$

PONTOS(TRECHOS) AREA SIGMA

FORCA SOMA TAU

C

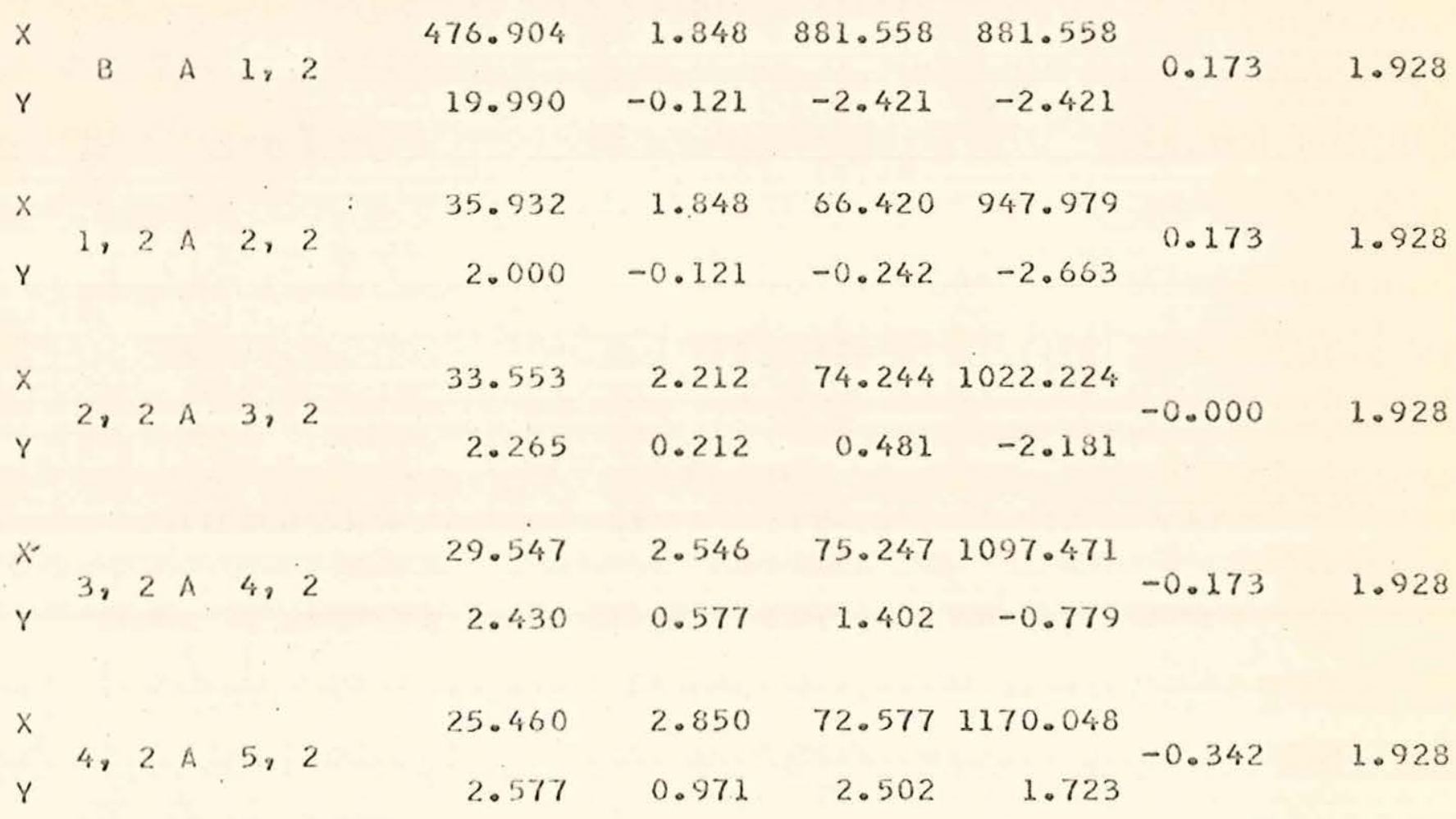




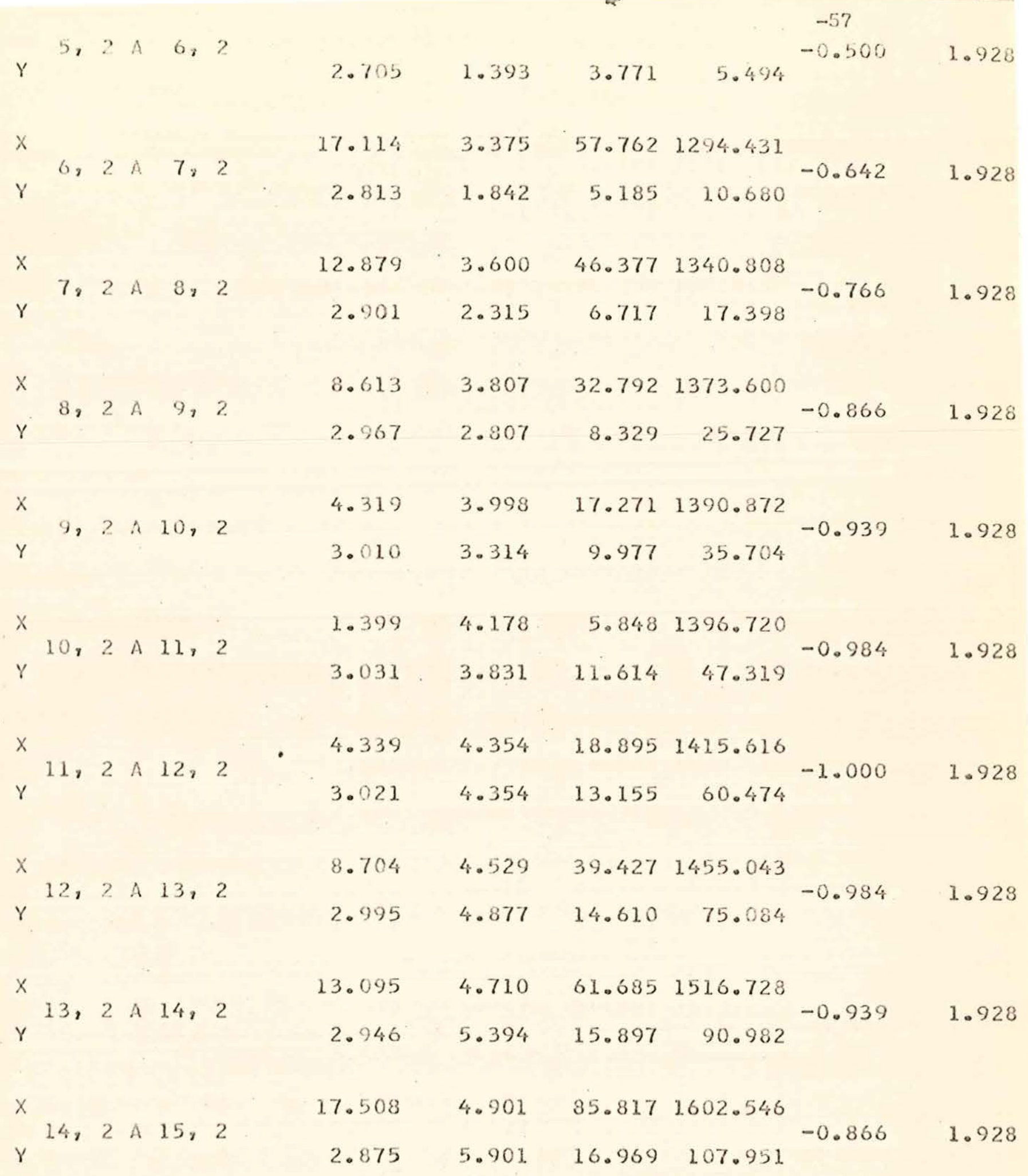

PARA A LINHA $J=3 \operatorname{COM~CI}=-0.863$

PONTOS(TRECHOS) AREA SIGMA FORCA SOMA TAU

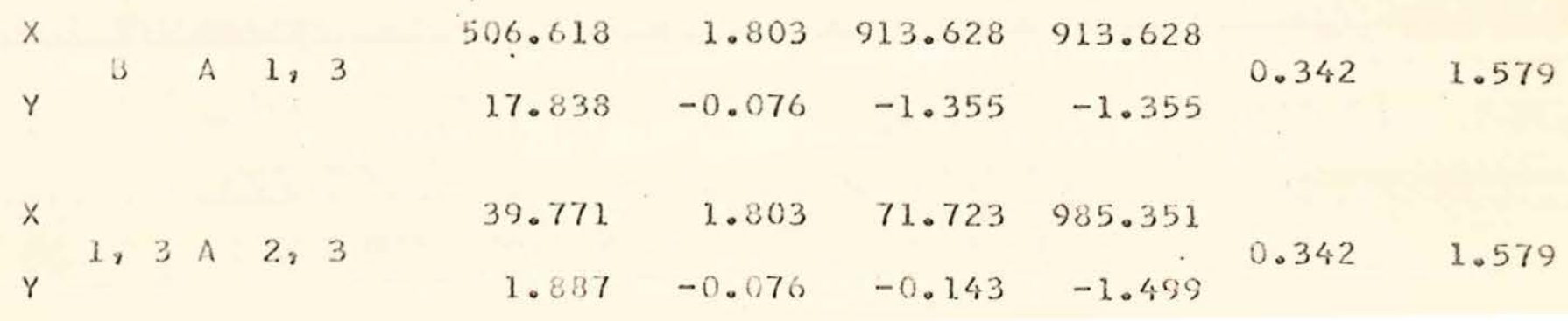




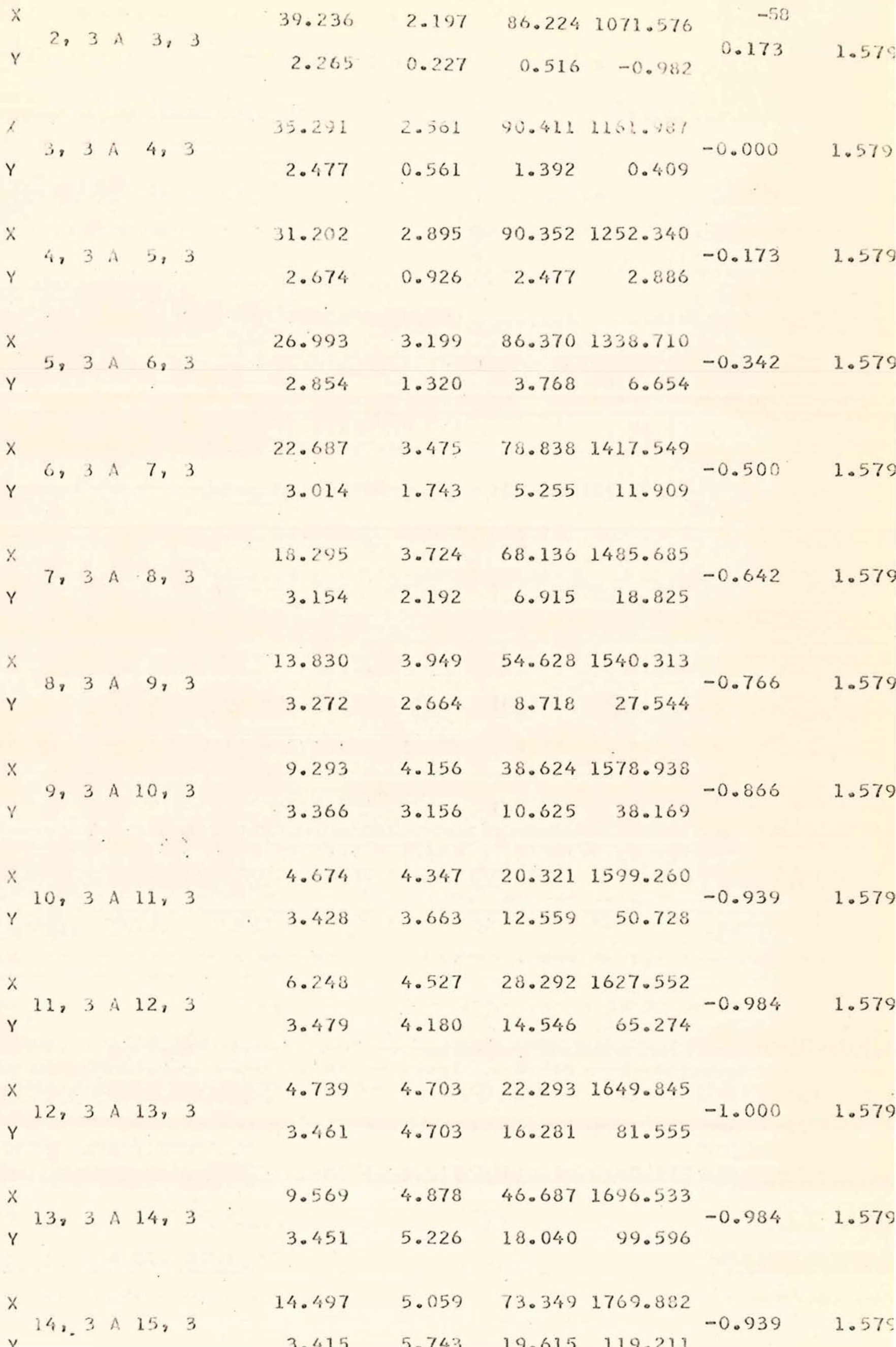


PARA A LINHA J $=4$ COM $\mathrm{C} 1=-0.863$

SIGMA

FORCA SOMA

TAU

C

$x$

B A 1,4

$532.484 \quad 1.729 \quad 921.046 \quad 921.046$

$\begin{array}{llll}15.550 & -0.002 & -0.036 & -0.036\end{array}$

0.499

1.230

Y

$x$

I, 4 A 2,4

$43.454 \quad 1.729 \quad 75.163 \quad 996.209$

$1.745 \quad-0.002 \quad-0.004 \quad-0.040$

0.499

1.230

Y

$44.980 \quad 2.152 \quad 96.818 \quad 1093.028$

2,4 A 3,4

2.217

0.273

0.605

0.342

1.230

Y

$41.160 \quad 2.546 \quad 104.820 \quad 1197.848$

$\times 3,4 \& 4,4$

$2.477^{\circ}$

0.577

1.429

1.994

0.173

1.230

37.127

2.910

108.0731305 .922

2.725

0.910

2.482

4.477

$-0.000$

1.230

$32.916 \quad 3.244 \quad 106.804 \quad 1412.727$

$5,4 A 6,4$

2.959

$1.27 \underline{5}$

3.773

8.251

$-0.173$

1.230

$28.555 \quad 3.548 \quad 101.336 \quad 1514.063$

$\mathrm{X}, 4 \times 7,4$

3.175

1.669

5.301

13.552

$-0.342$

1.230

$24.068 \quad 3.824 \quad 92.041 \quad 1606.105$

7,4 A 8,4

3.372

2.092

$-0.500$

1.230

$19.469 \quad 4.073 \quad 79.303 \quad 1685.408$

$\begin{array}{llll}3.548 & 2.541 \quad 9.016 \quad 29.623\end{array}$

$-0.642$

1.230

$\gamma$

$8,4 \therefore 9,4$

14.766

4.299

$63.481 \quad 1748.889$

3.699

3.013

11.149

$-0.766$

1.230

$\gamma$

9,4 A 10,4

9.940

4.505

$44.784 \quad 1793.673$

$\gamma$

$10,4 \mathrm{~A} 11,4$

3.818

3.505

13.386

54.159

$-0.866$

1.230 
$\gamma^{11,4 A 12,4}$

$\begin{aligned} & X \\ & Y\end{aligned} 12,4,13,4$

$\times 13,4,14,4$

$X 14,4 \times 15,4$
$3.890 \quad 4.012 \quad 15.608 \quad 69.767$

$-0.939$

1.230

$4.082 \quad 4.877 \quad 19.910 \quad 1837.051$

$\begin{array}{llll}3.994 & 4.529 & 18.095 & 37.863\end{array}$

$-0.984$

1.230

$5.167 \quad 5.052 \quad 26.107 \quad 1863.158$

$4.009 \quad 5.052^{20.260} \quad 208.123^{-1.000}$

1.230

$10.493 \quad 5.227 \quad 54.861 \quad 1918.020$

$\begin{array}{llll}4.018 & 5.575 & 22.401 & 130.525\end{array}$

$-0.984 \quad 1.230$

TAU

C

PARA A LINHA $J=5$ COH $C I=-0.863$

PONTOS(TRECHOS) AREA

SIGMA

FORCA SOMA

$554.441 \quad 1.629 \quad 903.594 \quad 903.594$

$\begin{array}{llll}13.143 & 0.097 & 1.283 & 1.283\end{array}$

0.642

0.881

Y

B. A 1,5$$
0.097
$$

46.957

1.629

76.527980 .121

1.575

0.097

0.153

1.437

0.642

0.881

50.722

$2.0 \% 8$

105.4401085 .562

2.120

0.346

0.735

2.172

0.499

0.881

$\begin{array}{llll}47.085 & 2.501 & 117.785 & 1203.347\end{array}$

2.426

0.622

1.509

3.682

0.342

0.881

$43.159 \quad 2.895 \quad 124.977 \quad 1328.324$

2.725

0.926

2.524

6.206

0.173

0.381

38.989

3.259

$127.103 \quad 1455.427$

3.014

1. 259

$3.797 \quad 10.003$

$-0.000$

0.881

$\gamma$

5,5 A 6. 5

34.616

3.593

$124.404 \quad 1579.831$

6,5 A 7,5

3.288

1.624

5.341

1.5 .345

$-0.173$

0.881

$30.073 \quad 3.897 \quad 117.218 \quad 1697.050$

$3.546 \quad 2.018$ 
$x$
$\mathrm{y} 85,29$

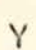

$9,5 \times 10,5$

$x$

10,5 A 11,5

Y

x

Y

$x$

$\gamma 12,5413,5$

X 13,5 A 14, 5

Y

$x$

$\gamma$

14,5 A 15,5
25.336

4.173

$105.943 \quad 1802.993$

3.785

2.441

$20.573 \quad 4.422$

4.001

2.890

90.9791893 .972

$11.565 \quad 43.310$

15.610

4.648

4.185

3.362

72.5591966 .531

14.072

57.383

10.491

4.854

50.9312017 .462

$4 \cdot 321$

3.854

16.654

74.038

$5.341 \quad 5.045 \quad 26.952 \quad 2044.415$

$4.463 \quad 4.361 \quad 19.467 \quad 93.506$

$11.466 \quad 5.226 \quad 59.9242104 .340$

$\begin{array}{llll}4.587 & 4.878 & 22.382 & 115.889\end{array}$

$-0.984$

$5.535 \quad 5.401 \quad 29.9022134 .242$

$\begin{array}{llll}4.580 & 5.401 & 24.741 & 140.630\end{array}$
$-0.939$

0.881

0.881

0.881

0.881

0.881

0.881

$-1.000$

0.881

PARA A LINHA $J=6$ COH CI $=-0.863$

PONTOS(TRECHOS) AREA STGMA

FORCA SOMA

TAU

C

$x$

B A 1,6

572.450

1.506

862.385

862.385

10.636

0.220

2.349

2.349

0.766

0.532

$\begin{array}{llll}50.260 & 1.506 & 75.715 & 938.101\end{array}$

$1,6 A^{\circ} 2,6$

1.376

0.220

0.304

0.766

0.532

Y

$x 2,6 \wedge 3,6$

56.404

1.978

111.6141049 .715

1.971

0.446

0.880

0.642

0.532

$\times, 6 A 4,6$
$Y$ A 4,6

.53 .007

2.427

128.6941178 .409

2.321

0.695

1.615

5.149

0.499

0.532 


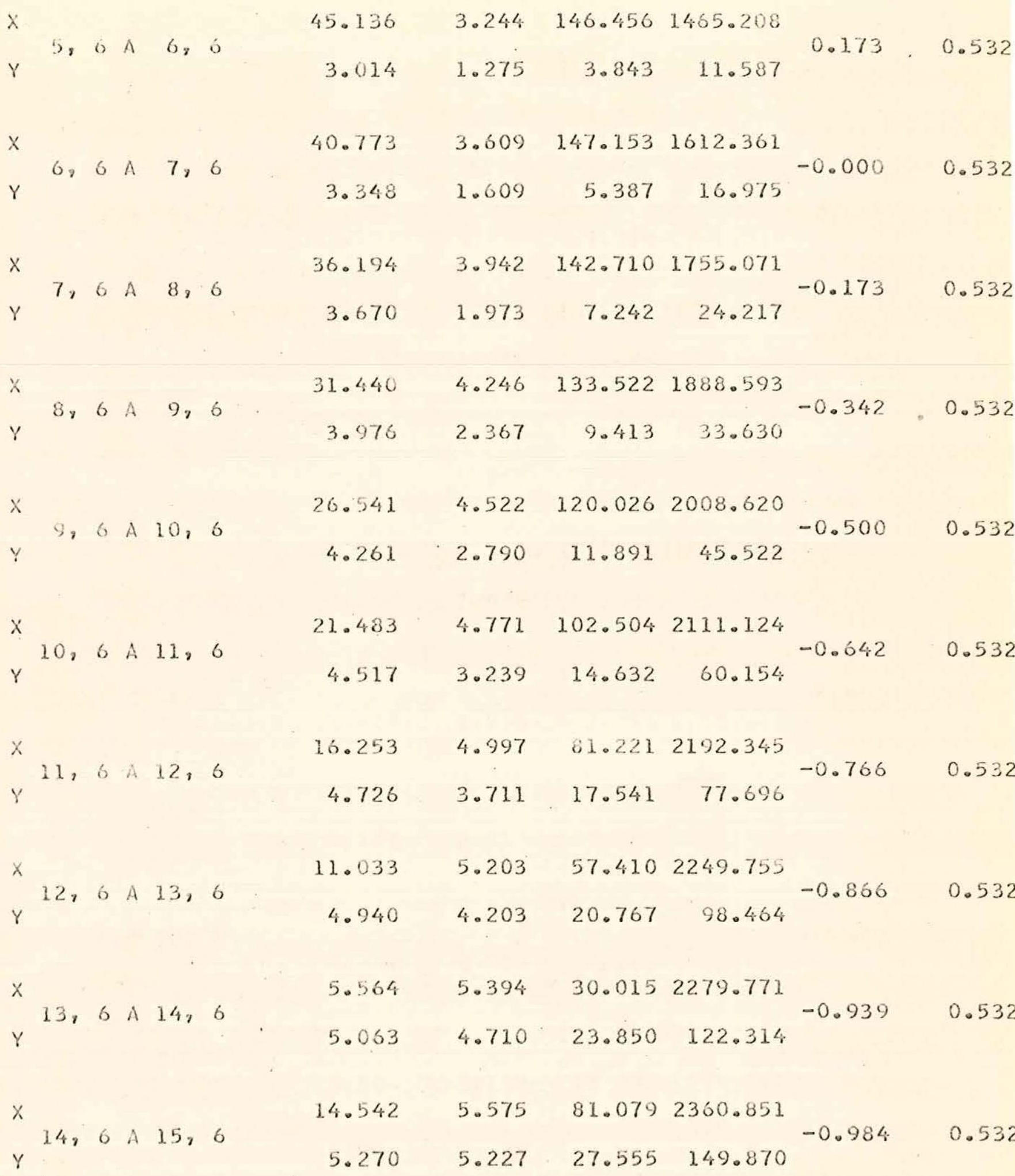

PARA A LINHA $J=7$ COM $C 1=-0.863$

PONTOS(TRECHOS) AREA SIGMA FORCA SOMA TAU

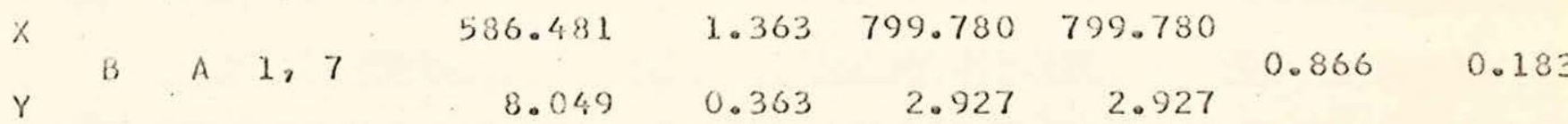


$1,7 \times 2,7$

$x \quad 2,7$ A 3,7

$x$

3,7 A 4,7

Y

x

4,7 A 5,7

Y

$x$

5,7 A 6,7

Y

$x$

$6,7 \AA 7,7$

Y

$\mathrm{x}$

7,7 A 3,7

Y.

$x$

$8,7 \wedge 9,7$

Y

$\times 3,7$ A 10,7

Y

$x$ Y

10,7 A 11,7

$X 11,7$ A 12, 7

Y

\begin{tabular}{l}
$X, 7$ A 13,7 \\
\hline
\end{tabular}

$\gamma$

$x$

13,7 A 14,7
1.150

0.363

0.418

3.345

0.866

$\begin{array}{llll}01.977 & 1.855 & 115.001 & 987.525\end{array}$

1.770

0.569

1.008

4.354

0.765

0.183

$\begin{array}{llll}58.876 & 2.327 & 137.057 & 1124.582\end{array}$

2.161

0.795

1.719

6.074

0.642

0.283

$\begin{array}{llll}55.292 & 2.776 & 153.543 & 1278.125\end{array}$

$\begin{array}{llll}2.557 & 1.044 & 2.672 & 8.746\end{array}$

0.499

0.183

$51.294 \quad 3.199 \quad 164.123 \quad 1442.249$

$2.954 \quad 1.320 \quad 3.900 \quad 12.647$

0.342

0.183

$46.951 \quad 3.593 \quad 168.734 \quad 1610.983$

$\begin{array}{llll}3.348 & 1.624 & 5.438 & 28.086\end{array}$

0.173

0.183

$\begin{array}{lllll}42.332 & 3.958 & 167.556 & 1778.540\end{array}$

$\begin{array}{llll}3.735 & 1.958 \quad 7.314 & 25.400\end{array}$

$-0.000$

0.183

$\begin{array}{llll}37.500 & 4.291 & 160.950 & 1939.490\end{array}$

$\begin{array}{llll}4.111 & 2.322 & 9.547 & 34.948\end{array}$

$-0.173$

0.183

$32.506 \quad 4.595 \quad 149.398 \quad 2088.889$

$\begin{array}{llll}4.471 & 2.716 & 12.146 \quad 47.094\end{array}$

$-0.342$

0.183

$27.352 \quad 4.871 \quad 133.243 \quad 2222.132$

$\begin{array}{llll}4.804 & 3.139 & 15.082 & 62.177\end{array}$

$-0.500$

0.183

$22.032 \quad 5.120 \quad 112.816 \quad 2334.948$

$\begin{array}{llll}5.094 & 3.588 & 18.279 & 80.456\end{array}$

$-0.642$

0.183

$16.781 \quad 5.346 \quad 89.717 \quad 2424.666$

$5.389 \quad 4.060 \quad 21.885 \quad 102.342$

$-0.766$

0.183

$11.272 \quad 5.552 \quad 62.593 \quad 2487.258$

5.594

$-0.366$

0.183 
$\begin{array}{r}x \\ y\end{array} 14,7 \wedge 15,7$
5.743

32.8572520 .116

$5.782 \quad 5.059 \quad 29.255 \quad 157.068$ $-0.939$

PARA A LINHA $J=8 \operatorname{COM} \mathrm{Cl}=-0.863$

PUNTOS(TRECHOS

$x$

B A 1,8

Y

$x$

$Y$

$1, \& A 2,8$

$x$

Y

2,8 A 3,8

$x$

$Y$

3,8 A 4,8

$x$

4,8 A 5,8

Y

$x$

Y

5,8 A 6,8

$x$

Y

6,8 A 7, 8

x

Y

7,8 A 8,8

$x$

Y

$8,8 \times 9,8$

x

Y

9,8 A 10,8

x

$10,8 \wedge 11,8$
AREA

596.514

5.400

0.521

2.817

2.817

56.190

1.205

0.897

0.521

67.749

0.468

3.285

67.393

1.712

1.516

0.712

115.428

902.403

1.0804 .366

64.649

1.943

2.204

0.919

1.786

6.152

61.297

2.676

$164.089 \quad 1209.018$

2.383

1.144

2.728

8.830

57.415

3.125

2.831

1.393

$179.479 \quad 1338.497$

$3.946 \quad 12.827$

53.089

3.540

18

3.283

1.669

5.481

1576.896

48.407

3.735

3.942

190.8671767 .763

1.973

7.372

25.679

43.458

4.182

4.307

$187.181 \quad 1954.944$

2.307

9.650

35.329

$-0.000-0.165$

38.320

4.641

177.845

2132.790

4.620

2.671

12.342

47.671

$-0.173-0.165$ 


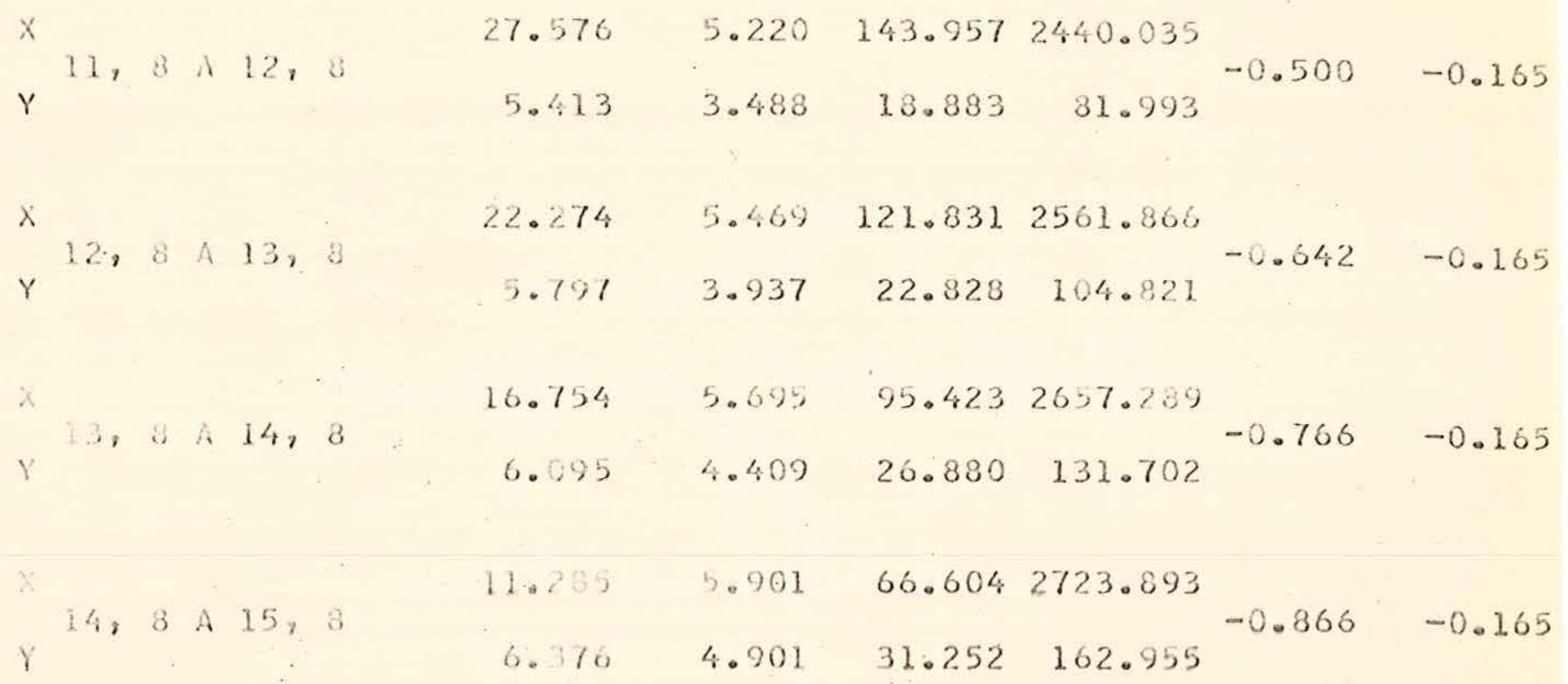

PARA A LIMHA $J=3$ GUM $C 1=-0.863$

PONTUS(TRECHOS) AREA SYGMA FORCA SOMA TAU T

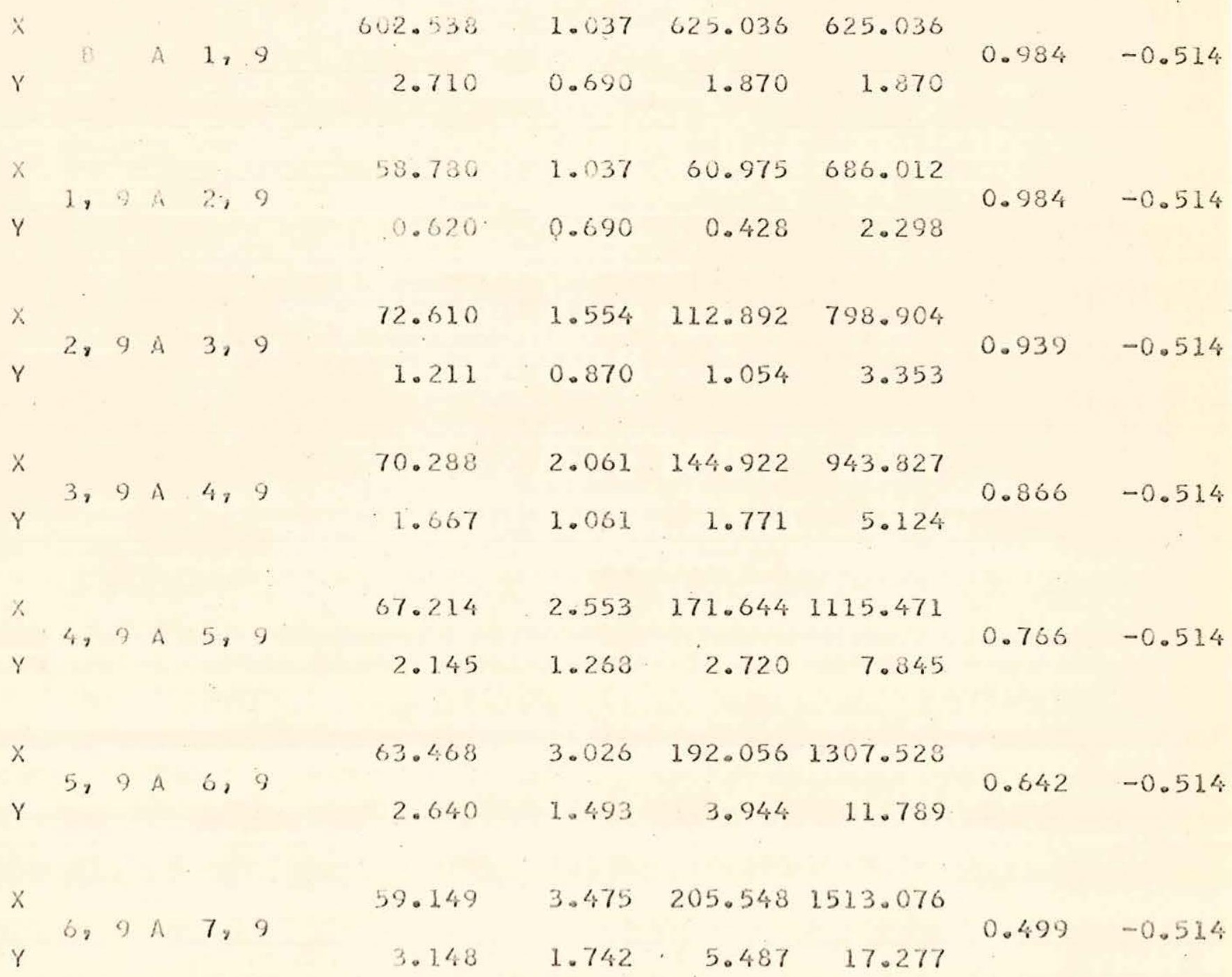




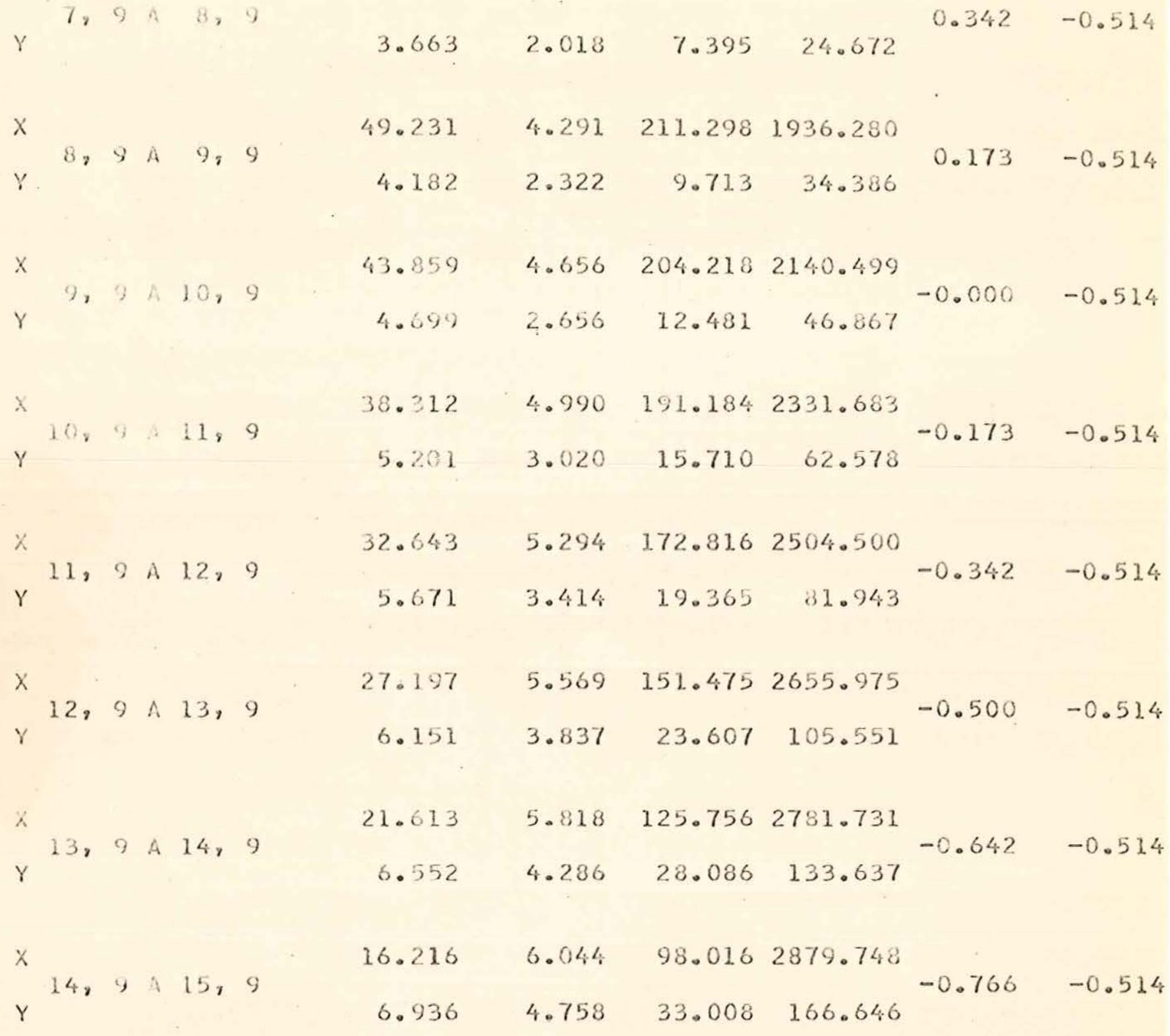

PARA A LINHA $J=10$ COM CI $=-0.863$

PONTOS (TRECHOS) AREA

FORCA SOMA

TAU

C

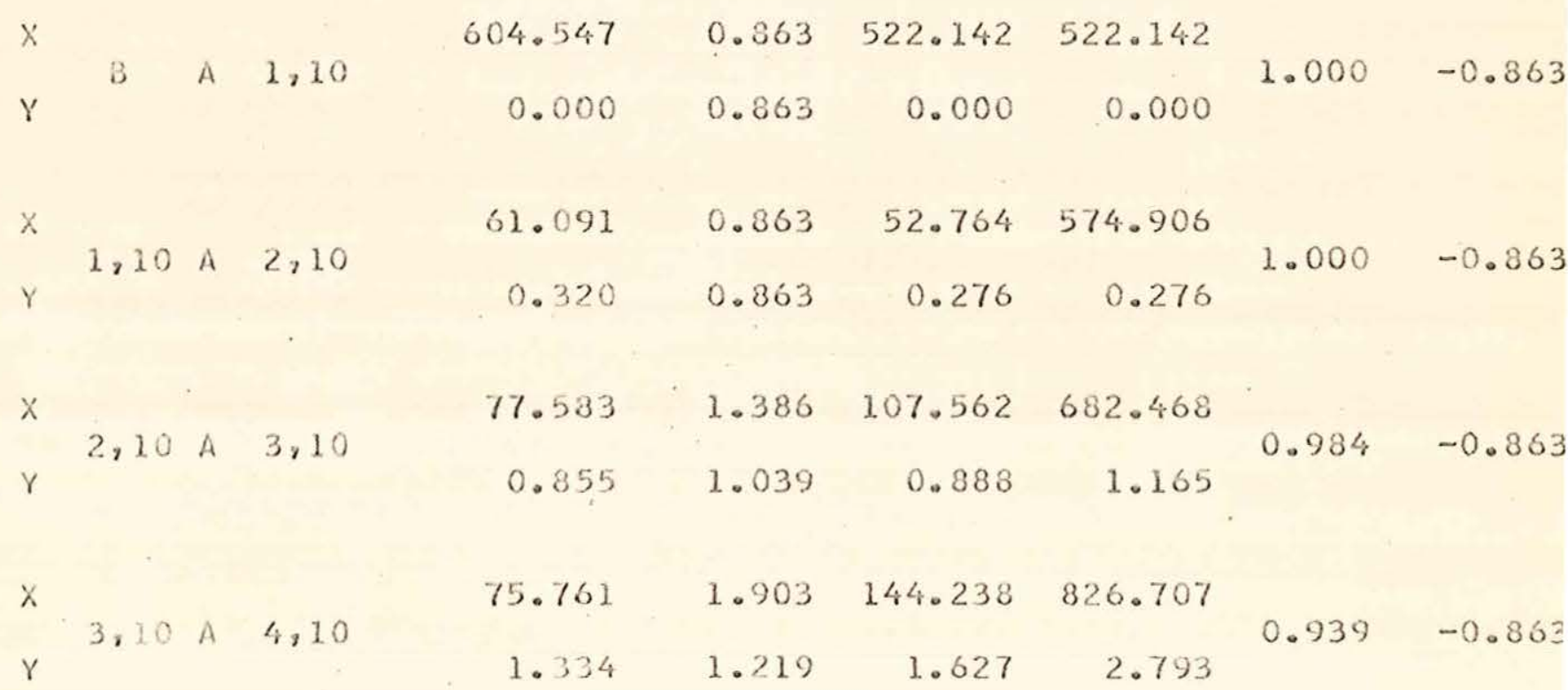




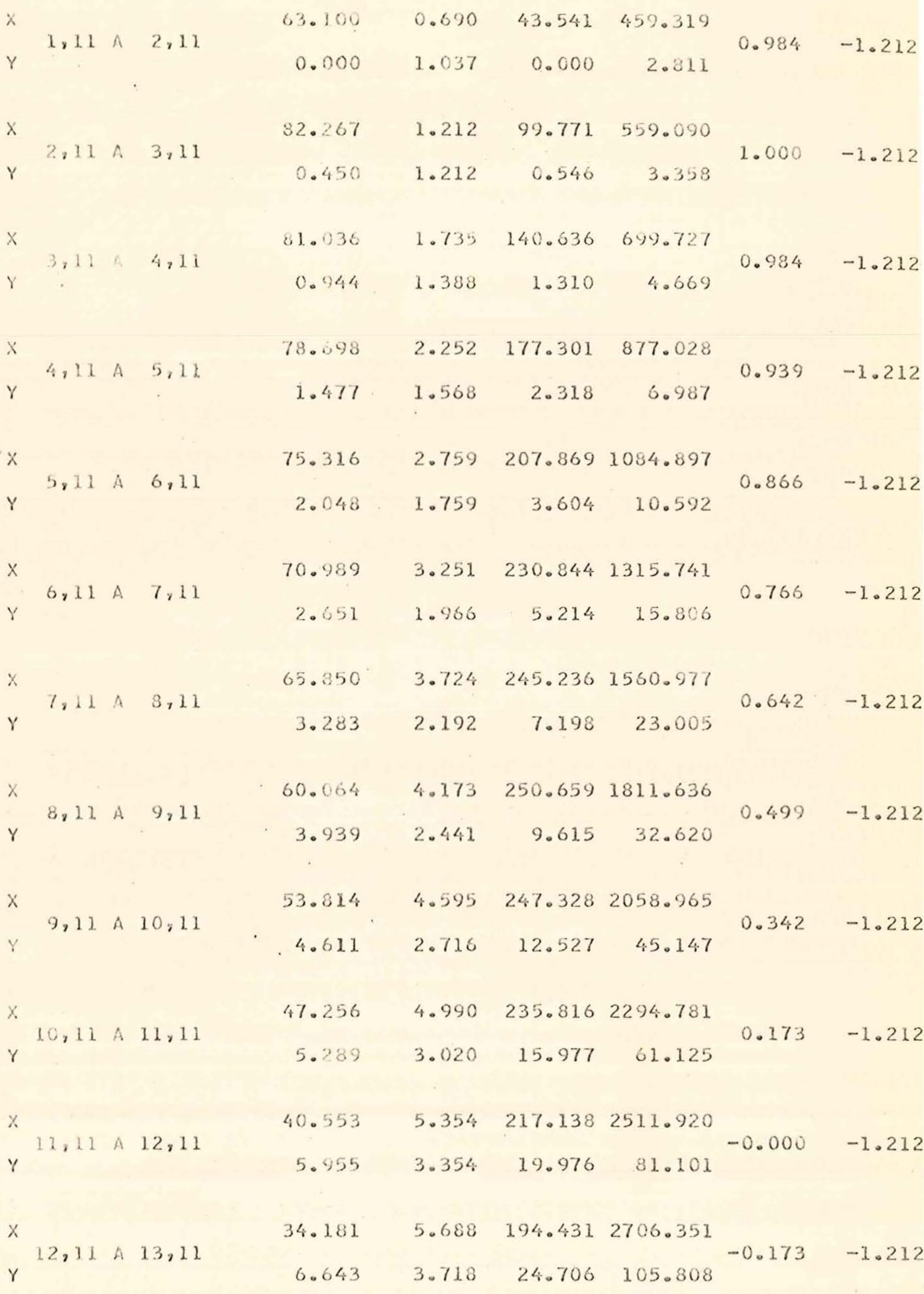


13,11 A 14,11

$\gamma$
$\times 14,11 \wedge 15,11$

$\begin{array}{llll}1.271 & 4.112 \quad 29.908 \quad 135.716\end{array}$

$-0.342-1.212$

$22.142 \quad 6.267 \quad 138.780 \quad 3012.256$

$\begin{array}{llll}7.893 & 4.535 & 35.798 & 171.515\end{array}$

$-0.500-1.212$

PARA A LINHA $\mathrm{J}=12$ COM $\mathrm{CI}=-0.863$

PONTOS(TRECHOS) AREA SIGMA FORCA SOMA TAU C

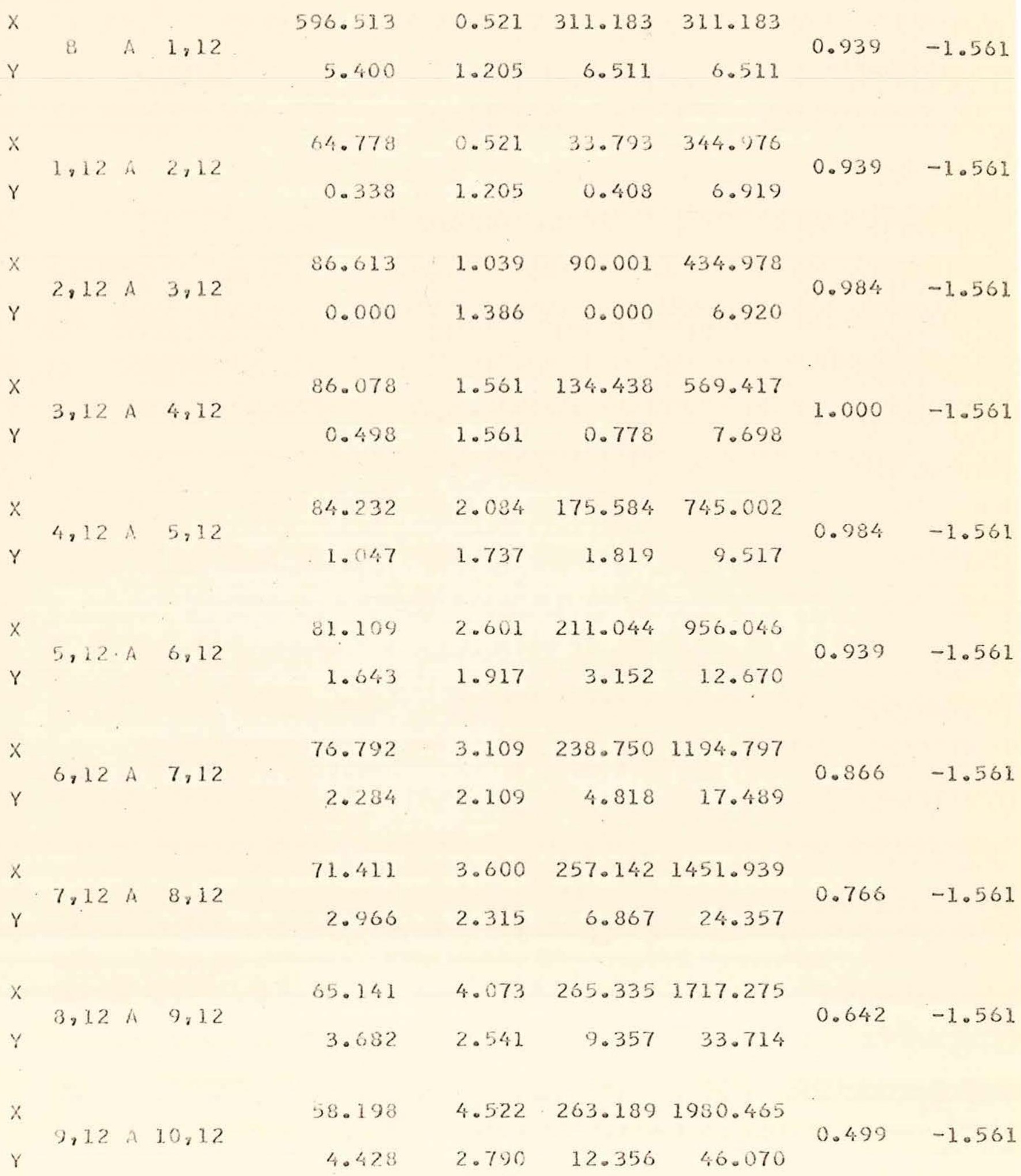




\begin{tabular}{|c|c|c|c|c|c|c|c|}
\hline & & 50.785 & 4.944 & 251.131 & & & \\
\hline 10,12 & 111,12 & 5.192 & 3.065 & 15.918 & 61.989 & 0.342 & -1.561 \\
\hline 11,12 & A 12,12 & $\begin{array}{r}43.132 \\
5.957\end{array}$ & $\begin{array}{l}5.339 \\
3.369\end{array}$ & $\begin{array}{r}230.293 \\
20.074\end{array}$ & $\begin{array}{r}2461.889 \\
82.063\end{array}$ & 0.173 & -1.561 \\
\hline 12,12 & A 13,12 & $\begin{array}{r}35.789 \\
6.754\end{array}$ & $\begin{array}{l}5.703 \\
3.703\end{array}$ & $\begin{array}{r}204.123 \\
25.013\end{array}$ & $\begin{array}{r}2666.012 \\
107.076\end{array}$ & -0.000 & -1.561 \\
\hline 13,12 & A 14,12 & $\begin{array}{r}28.614 \\
7.504\end{array}$ & $\begin{array}{l}6.037 \\
4.067\end{array}$ & $\begin{array}{r}172.754 \\
30.524\end{array}$ & $\begin{array}{r}2838.766 \\
137.601\end{array}$ & -0.273 & -1.5 \\
\hline 14,12 & A 15,12 & $\begin{array}{l}22.155 \\
8.255\end{array}$ & $\begin{array}{l}6.341 \\
4.461\end{array}$ & $\begin{array}{r}140.491 \\
36.833\end{array}$ & $\begin{array}{r}2979.257 \\
174.435\end{array}$ & -0.342 & -1.56 \\
\hline
\end{tabular}

PARA A LINHA J=13 COM CLI $=-0.863$

PONTOS(TRECHGS) AREA SIGMA FORCA SUMA TAU O

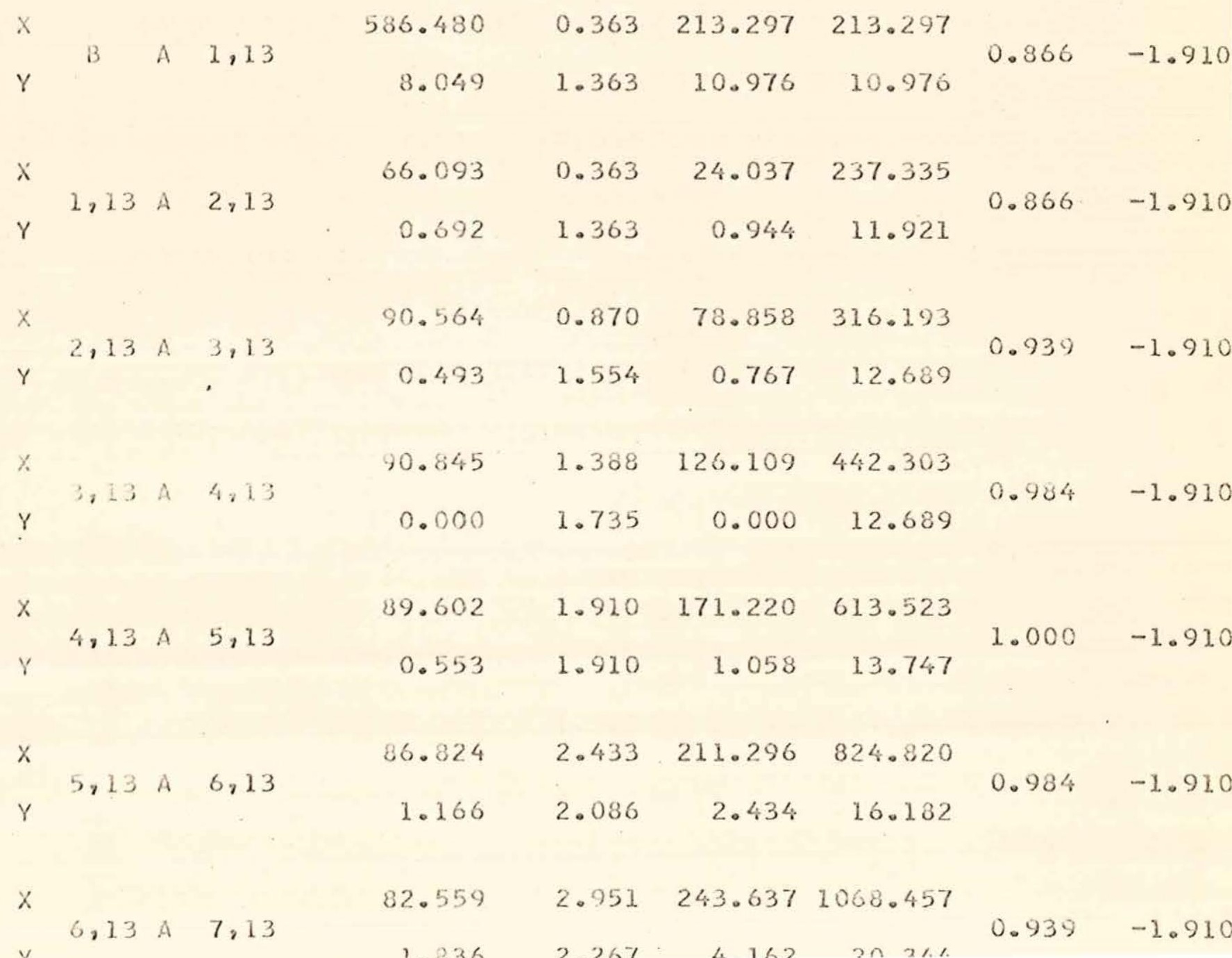




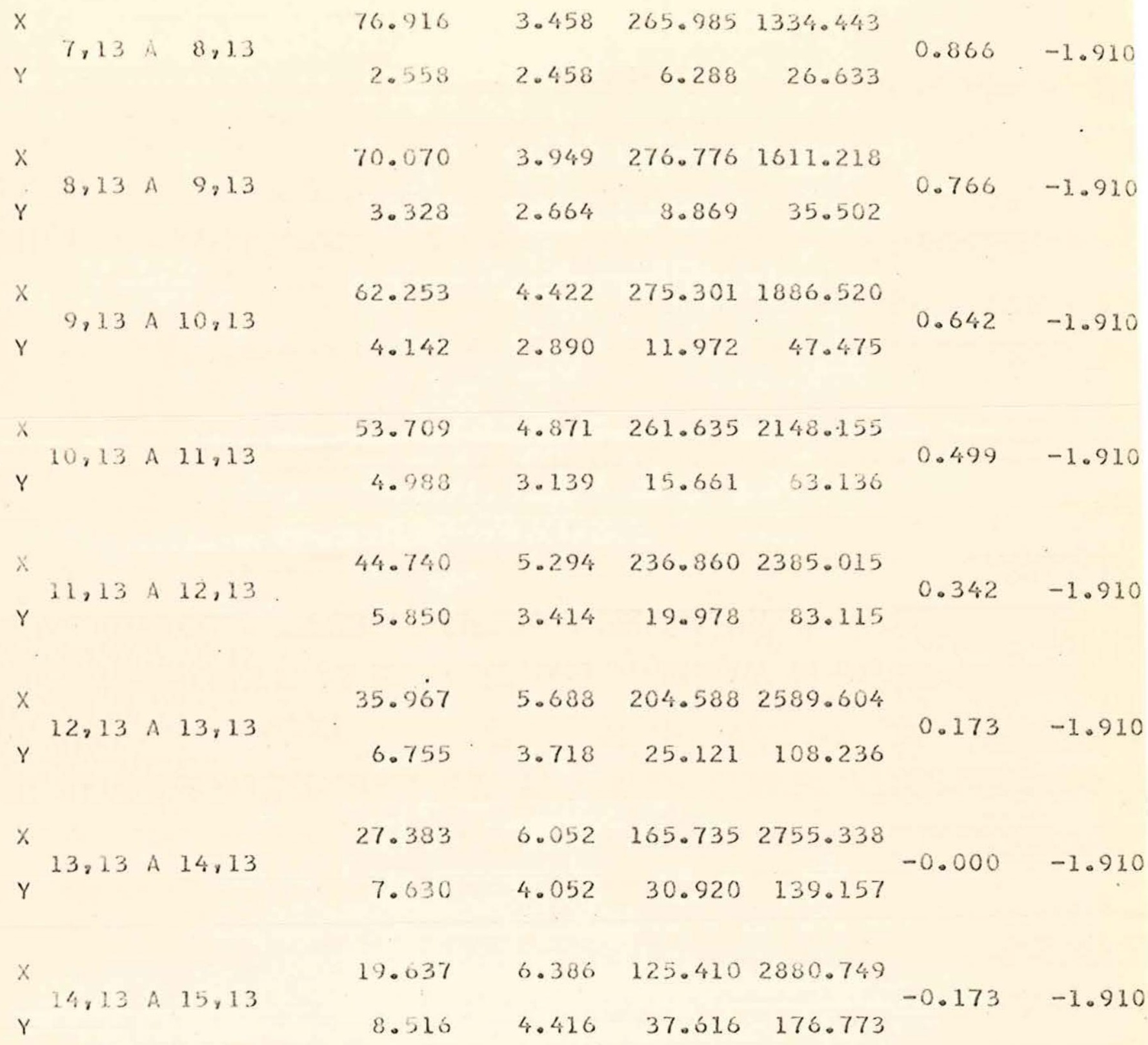

PARA A LINHA $\mathrm{J}=14$ COM $\mathrm{Cl}=-0.863$

PONTOS(TRECHDS) AREA SIGMA. FORCA SOMA TAU C

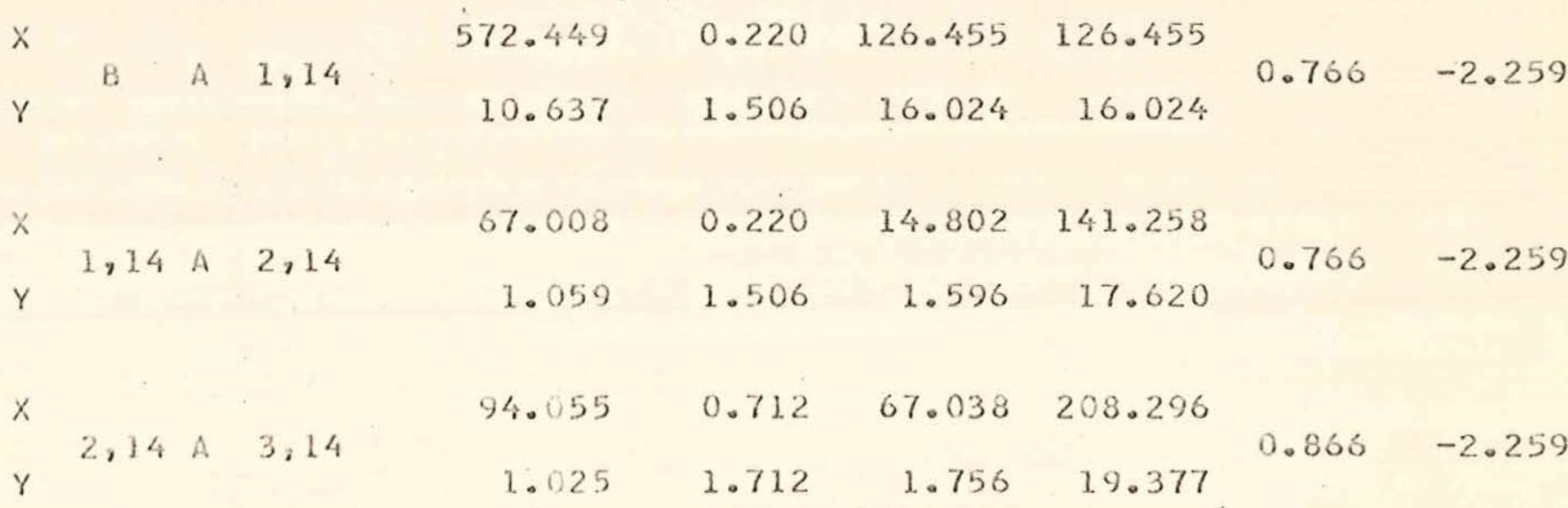


3,14 A 4, 14

$r$

$X$
$Y$

$X$
$Y$

$x \quad 6,14 \wedge 7,14$

Y

$x$
$y$ $7.14 \mathrm{~A} \quad 8.14$

$x$

Y 8,14 A 9,14

$x$

Y

9,14 A 10,14

$x$

Y

10,14 A 11,14

$\times 11,14$ A 12,14

$\gamma$

$x^{x} 12,14 \wedge 13,14$

$\begin{array}{r}x \\ y\end{array} 13,14$ A 14,14

$X$
$Y$
$Y, 14 \wedge 15,14$
$0.548 \quad 1.903 \quad 1.043 \quad 20.420$

$94.785 \quad 1.737 \quad 164.664 \quad 489.195$

$0.000 \quad 2.084 \quad 0.000 \quad 20.420$

$92.468 \quad 2.259 \quad 208.974 \quad 698.169$

$\begin{array}{llll}0.613 & 2.259 & 1.397 & 21.818\end{array}$

$1.000-2.259$

$\begin{array}{llll}83.332 & 2.782 & 245.799 & 943.968\end{array}$

$1.305 \quad 2.435 \quad 3.178 \quad 24.997$

$82.444 \quad 3.300 \quad 272.074 \quad 1216.042$

$2.058 \quad 2.616 \quad 5.385 \quad 30.382$

$0.939-2.259$

$\begin{array}{llll}74.955 & 3.807 & 285.367 & 1501.410\end{array}$

$\begin{array}{llll}2.874 & 2.807 & 8.068 & 38.450\end{array}$

$0.866-2.259$

$66.096 \quad 4.299 \quad 284.150 \quad 1785.560$

$3.747 \quad 3.013 \quad 11.293 \quad 49.743$

$0.766-2.259$

$56.139 \quad 4.771 \quad 267.862 \quad 2053.422$

$\begin{array}{llll}4.669 & 3.239 & 15.126 & 64.870\end{array}$

$0.642-2.259$

$45.448 \quad 5.220 \quad 237.2592290 .682$

$5.624 \quad 3.488 \quad 19.620 \quad 84.490$

$0.499-2.259$

$34.709 \quad 5.643 \quad 195.8692486 .551$

$6.634 \quad 3.763 \quad 24.972 \quad 109.462$

$0.342-2.259$

$24.069 \quad 6.037 \quad 145.3172631 .868$

$\begin{array}{llll}7.634 & 4.067 \quad 31.054 & 140.516\end{array}$

$0.173-2.259$

$1.4 .292 \quad 6.401 \quad 91.497 \quad 2723.365$

$8.659 \quad 4.401 \quad 38.114 \quad 178.631^{-0.000} \quad-2.259$

PARA A LINHA J=15 COM CI $=-0.863$ 


\begin{tabular}{|c|c|c|c|c|c|c|c|c|c|}
\hline$x$ & & & & 554.440 & 0.097 & 54.138 & 54.138 & -73 & \\
\hline$\gamma$ & 1) & A & 1,15 & 13.143 & 1.629 & 21.420 & 21.420 & 0.642 & -2.60 \\
\hline$\gamma$ & 1,15 & A & 2,15 & $\begin{array}{l}67.480 \\
1.435\end{array}$ & $\begin{array}{l}0.097 \\
1.629\end{array}$ & $\begin{array}{l}6.589 \\
2.338\end{array}$ & $\begin{array}{l}60.727 \\
23.759\end{array}$ & 0.642 & -2.60 \\
\hline & 2,10 & $A$ & 3,15 & $\begin{array}{l}97.009 \\
1.592\end{array}$ & $\begin{array}{l}0.569 \\
1.855\end{array}$ & $\begin{array}{r}55.292 \\
2.954\end{array}$ & $\begin{array}{l}116.020 \\
26.713\end{array}$ & 0.766 & -2.60 \\
\hline & 3,15 & A & 4,15 & $\begin{array}{r}99.345 \\
1.142\end{array}$ & $\begin{array}{l}1.061 \\
2.061\end{array}$ & $\begin{array}{r}105.487 \\
2.354\end{array}$ & $\begin{array}{l}221.507 \\
29.068\end{array}$ & 0.866 & -2.60 \\
\hline & 4,15 & A & 5,15 & $\begin{array}{l}99.741 \\
0.611\end{array}$ & $\begin{array}{l}1.568 \\
2.252\end{array}$ & $\begin{array}{r}156.481 \\
1.377\end{array}$ & $\begin{array}{r}377.989 \\
30.446\end{array}$ & 0.939 & -2.60 \\
\hline Y & 5,15 & A & 6,15 & $\begin{array}{l}98.033 \\
0.000\end{array}$ & $\begin{array}{l}2.086 \\
2.433\end{array}$ & $\begin{array}{r}204.537 \\
0.000\end{array}$ & $\begin{array}{r}582.527 \\
30.446\end{array}$ & 0.984 & -2.60 \\
\hline Y & 6,15 & A & 7.15 & $\begin{array}{l}94.151 \\
0.692\end{array}$ & $\begin{array}{l}2.609 \\
2.609\end{array}$ & $\begin{array}{r}245.642 \\
1.807\end{array}$ & $\begin{array}{r}828.169 \\
32.253\end{array}$ & 1.000 & -2.60 \\
\hline$Y$ & 7,15 & $A$ & 8,15 & $\begin{array}{r}88.081 \\
1.465\end{array}$ & $\begin{array}{l}3.131 \\
2.784\end{array}$ & $\begin{array}{r}275.849 \\
4.079\end{array}$ & $\begin{array}{r}1104.019 \\
36.333\end{array}$ & 0.984 & -2.60 \\
\hline $\begin{array}{l}X \\
Y\end{array}$ & 8,15 & $A$ & 9,15 & $\begin{array}{r}79.929 \\
2.315\end{array}$ & $\begin{array}{l}3.649 \\
2.965\end{array}$ & $\begin{array}{r}291.677 \\
6.864\end{array}$ & $\begin{array}{r}1395.696 \\
43.197\end{array}$ & 0.939 & -2.60 \\
\hline y & 9,15 & $A$ & 10,15 & $\begin{array}{l}69.897 \\
3.238\end{array}$ & $\begin{array}{l}4.156 \\
3.156\end{array}$ & $\begin{array}{r}290.511 \\
10.221\end{array}$ & $\begin{array}{r}1686.207 \\
53.419\end{array}$ & 0.866 & -2.60 \\
\hline $\begin{array}{l}X \\
\gamma\end{array}$ & $10,1.5$ & $A$ & 11,15 & $\begin{array}{r}58.261 \\
4.227\end{array}$ & $\begin{array}{l}4.648 \\
3.362\end{array}$ & $\begin{array}{r}270.802 \\
14.216\end{array}$ & $\begin{array}{r}1957.010 \\
67.635\end{array}$ & 0.766 & -2.60 \\
\hline $\begin{array}{l}X \\
Y\end{array}$ & 11,15 & A & 12,15 & $\begin{array}{r}45.427 \\
5.268\end{array}$ & $\begin{array}{l}5.120 \\
3.588\end{array}$ & $\begin{array}{r}232.606 \\
18.904\end{array}$ & $\begin{array}{r}2189.616 \\
86.540\end{array}$ & 0.642 & -2.60 \\
\hline \% & 12.15 & $A$ & 13,15 & 32.135 & 5.569 & 178.975 & 2368.591 & 0.499 & 2.6 \\
\hline
\end{tabular}




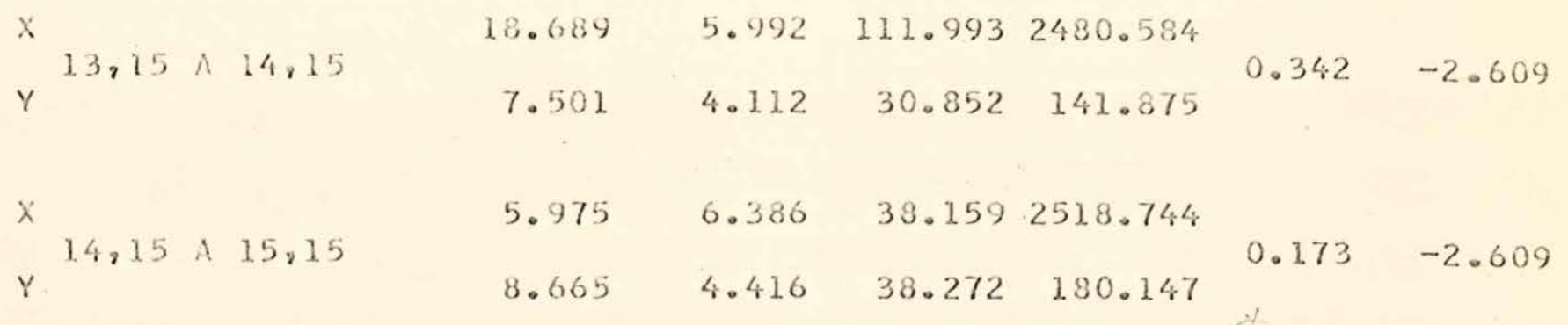

HODOGRAFO

PONTO X(CM) $Y(C M) \quad M O O V$

$\begin{array}{lrr}A & 3.525 & 21.913 \\ B & -3.474 & 21.913\end{array}$

$\begin{array}{llll}15.15 & 0.025 & 28.413 & 4.949\end{array}$

$15,14 \quad-0.292 \quad 18.121 \quad 4.949$

$25.13-0.635 \quad 17.858 \quad 4.949$

$15,12 \quad-0.999 \quad 17.626 \quad 4.949$

$\begin{array}{llll}15.11 & -1.382 & 17.427 & 4.949\end{array}$

$15.10 \quad-1.781 \quad 17.262 \quad 4.949$

$15.9 \quad-2.193 \quad 17.132 \quad 4.949$

$15,8 \quad-2.614 \quad 17.038 \quad 4.949$

$15,7 \quad-3.042 \quad 16.932 \quad 4.949$

$15.6 \quad-3.474 \quad 16.963 \quad 4.949$

$15,5 \quad-3.905 \quad 16.982 \quad 4.949$

$15,4 \quad-4.333 \quad 17.038 \quad 4.949$

$15.3 \quad-4.755 \quad 17.132 \quad 4.949$

$15,2 \quad-5.167 \quad 17.262 \quad 4.949$

$15,1 \quad-5.566 \quad 17.427 \quad 4.949$

$\begin{array}{llll}14225 & 0.344 & 18.121 & 5.381\end{array}$

$14.14 \quad 0.025 \quad 17.803 \quad 5.398$

$14.13 \quad-0.334 \quad 17.500 \quad 5.415$

$14.12-0.721 \quad 17.230 \quad 5.432$

$\begin{array}{llll}14.11 & -1.131 & 16.993 & 5.449\end{array}$

$14,10 \quad-1.562 \quad 16.792 \quad 5.466$ 


\begin{tabular}{|c|c|c|c|}
\hline 14,8 & -2.471 & 16.505 & 5.499 \\
\hline 14,7 & -2.944 & 16.422 & 5.516 \\
\hline 4,6 & -3.423 & 16.380 & 5.533 \\
\hline 14,5 & -3.905 & 16.380 & 5.549 \\
\hline 24,4 & -4.387 & 16.422 & 5.566 \\
\hline 14,3 & -4.805 & 10.507 & 5.582 \\
\hline $1<, 2$ & -5.335 & 26.633 & 5.599 \\
\hline 14,1 & -5.794 & 16.300 & 5.615 \\
\hline 13,15 & 0.686 & 17.858 & 5.809 \\
\hline 13,14 & 0.386 & 17.500 & 5.863 \\
\hline 13,13 & 0.025 & 17.140 & 5.918 \\
\hline 13,12 & -0.368 & 16.809 & 5.974 \\
\hline 13,11 & -0.794 & 16.511 & 6.030 \\
\hline $3.3,10$ & -1.248 & 16.249 & 6.085 \\
\hline 13,9 & -1.728 & 16.025 & 6.141 \\
\hline 13,8 & -2.230 & 15.842 & 6.196 \\
\hline 13,7 & -2.751 & 15.703 & 6.252 \\
\hline 13,6 & -3.287 & 15.608 & 6.307 \\
\hline 13,5 & -3.834 & 15.560 & 6.362 \\
\hline 13,4 & -4.387 & 15.560 & 6.418 \\
\hline 13,3 & -4.944 & 15.609 & 6.473 \\
\hline 13,2 & -5.499 & 15.707 & 6.528 \\
\hline 13,1 & -6.047 & 15.854 & 6.583 \\
\hline 12,15 & 1.050 & 17.626 & 6.233 \\
\hline 12,14 & 0.772 & 17.230 & 6.322 \\
\hline 12,13 & 0.420 & 16.8309 & 6.420 \\
\hline 12,12 & 0.025 & 16.415 & 6.517 \\
\hline 12,11 & -0.408 & 16.051 & 6.615 \\
\hline 12,10 & -0.379 & 15.721 & 6.714 \\
\hline 12,9 & -1.384 & 15.429 & 6.812 \\
\hline 2,8 & -1.921 & $15.17 \%$ & 6.911 \\
\hline
\end{tabular}




\begin{tabular}{|c|c|c|c|}
\hline 12,7 & -2.485 & 14.973 & 7.009 \\
\hline 12,6 & -3.074 & 14.315 & 7.108 \\
\hline 12,5 & -3.693 & 14.708 & 7.207 \\
\hline 12,4 & -4.308 & 14.653 & 7.307 \\
\hline 12,3 & -4.944 & 14.653 & 7.406 \\
\hline 12,2 & -5.586 & 14.710 & 7.506 \\
\hline 12,1 & -6.229 & 14.823 & 7.606 \\
\hline 11,15 & 1.433 & 17.427 & 6.649 \\
\hline 11,14 & 1.163 & 16.993 & 6.774 \\
\hline 11,13 & 0.845 & 16.511 & 6.917 \\
\hline 11,12 & 0.459 & 16.051 & 7.059 \\
\hline 11,11 & 0.025 & 15.617 & 7.203 \\
\hline 11,10 & -0.454 & 15.214 & 7.348 \\
\hline 11,9 & -0.977 & 14.848 & 7.493 \\
\hline 11,8 & -1.541 & 14.522 & 7.639 \\
\hline 11,7 & -2.144 & 14.241 & 7.786 \\
\hline 11,6 & -2.781 & 14.009 & 7.934 \\
\hline 11,5 & -3.448 & 13.830 & 8.082 \\
\hline 11,4 & -4.141 & 13.708 & 8.232 \\
\hline 11,3 & -4.856 & 13.646 & 8.382 \\
\hline $11: 2$ & -5.586 & 13.646 & 8.532 \\
\hline 11,1 & -6.327 & 13.710 & 8.684 \\
\hline 10,15 & 1.832 & 17.262 & 7.056 \\
\hline 10,14 & 1.613 & 16.792 & 7.218 \\
\hline 10,13 & 1.299 & 16.249 & 7.408 \\
\hline 10,12 & 0.930 & 15.721 & 7.599 \\
\hline 10,11 & 0.505 & 15.214 & 7.791 \\
\hline 10,10 & 0.025 & 14.735 & 7.986 \\
\hline 10,9 & -0.507 & 14.287 & 8.182 \\
\hline 10,8 & -1.091 & 13.878 & 8.360 \\
\hline $\mathrm{LO}_{2} 7$ & -1.724 & 13.513 & 8.580 \\
\hline
\end{tabular}




$$
\begin{array}{llll}
10,5 & -3.122 & 12.935 & 8.985 \\
10,4 & -3.880 & 12.732 & 9.190 \\
10,3 & -4.670 & 12.592 & 9.397
\end{array}
$$$$
10,2 \quad-5.437 \quad 12.521 \quad 9.605
$$$$
10,1 \quad-6.327 \quad 12.521 \quad 9.815
$$$$
\begin{array}{llll}
7.15 & 2.244 & 17.232 & 7.454
\end{array}
$$$$
9.14 \quad 2.061 \quad 16.629 \quad 7.652
$$$$
9,13 \quad 1.769 \quad 16.025 \quad 7.891
$$$$
\begin{array}{llll}
9.12 & 1.435 & 15.429 & 3.133
\end{array}
$$$$
9.11 \quad 1.028 \quad 14.848 \quad 8.378
$$$$
9,10 \quad 0.558 \quad 14.287 \quad 8.626
$$$$
9, \quad 9 \quad 0.025 \quad 13.754 \quad 8.377
$$$$
9,8 \quad-0.568 \quad 13.256 \quad 9.131
$$$$
9,7 \quad-1.223 \quad 12.797 \quad 9.389
$$$$
\begin{array}{lllll}
9.6 & -1.934 & 12.387 & 9.650
\end{array}
$$$$
\begin{array}{llll}
9.5 & -2.700 & 12.030 & 9.913
\end{array}
$$$$
3=\quad-3.516 \quad 11.733 \quad 10.180
$$$$
9,3 \quad-4.378 \quad 11.502 \quad 10.450
$$$$
9.2 \quad-5.280 \quad 11.343 \quad 10.723
$$$$
9,1 \quad-6.216 \quad 11.261 \quad 10.999
$$$$
\begin{array}{llll}
8.15 & 2.666 & 17.038 & 7.840
\end{array}
$$$$
8.14 \quad 2.523 \quad 16.505 \quad 8.075
$$$$
8,13 \quad 2.281 \quad 15.842 \quad 3.366
$$$$
\begin{array}{llll}
3.12 & 1.972 & 15.179 & 8.661
\end{array}
$$$$
8.11 \quad 1.503 \quad 14.522 \quad 8.961
$$$$
8,10 \quad 1.142 \quad 13.378 \quad 9.266
$$

$390.620 \quad 13.256 \quad 9.576$

$\begin{array}{llll}3,8 & 0.025 & 12.661 & 9.891\end{array}$

$8,7 \quad-0.640 \quad 12.102 \quad 10.211$

$8.6 \quad-1.375 \quad 11.588 \quad 10.536$

$8,5 \quad-2.177 \quad 11.124 \quad 10.866$ 


$$
\begin{aligned}
& 3,4 \quad-3.044 \quad 10.720 \quad 11.201 \\
& \begin{array}{lllll}
87 & -3.910 & 20.383 & 11.540
\end{array} \\
& 8,2 \quad-4.952 \quad 10.120 \quad 11.885 \\
& 8,1 \quad-5.983 \quad 9.938 \quad 12.234 \\
& \begin{array}{llll}
7.15 & 3.094 & 16.982 & 8.213
\end{array} \\
& \begin{array}{llll}
7.14 & 2.995 & 16.422 & 8.485
\end{array} \\
& \begin{array}{llll}
7.13 & 2.802 & 15.703 & 8.830
\end{array} \\
& \begin{array}{llll}
7.12 & 2.537 & 14.973 & 9.181
\end{array} \\
& \begin{array}{llll}
7.11 & 2.195 & 14.241 & 9.540
\end{array} \\
& \begin{array}{llll}
7.10 & 1.715 & 13.513 & 9.905
\end{array} \\
& \begin{array}{llll}
7.9 & 1.274 & 12.797 \quad 10.278
\end{array} \\
& \begin{array}{lllll}
7,8 & 0.691 & 12.102 & 10.658
\end{array} \\
& \begin{array}{llll}
7.7 & 0.025 & 11.437 & 11.045
\end{array} \\
& \begin{array}{lllll}
7.6 & -0.722 & 10.809 & 11.439
\end{array} \\
& \begin{array}{llll}
7,5 & -1.550 & 10.229 & 11.841
\end{array} \\
& \begin{array}{lllll}
7.4 & -2.458 & 9.705 & 12.250
\end{array} \\
& \begin{array}{lllll}
7.3 & -3.441 & 9.247 & 12.566
\end{array} \\
& \begin{array}{lllll}
7,2 & -4.495 & 8.863 & 13.089
\end{array} \\
& \text { 7.1. }-5.615 \quad 8.563 \quad 13.520 \\
& 6,15 \quad 3.525 \quad 16.963 \quad 8.573 \\
& 6.14 \quad 3.474 \quad 16.380 \quad 8.882 \\
& \begin{array}{llll}
6.13 & 3.338 & 15.608 & 9.282
\end{array} \\
& 6,12 . \quad 3.126 \quad 14.815 \quad 9.692 \\
& \begin{array}{llll}
6.11 & 2.832 & 14.009 & 10.111
\end{array} \\
& 6.10 \quad 2.453 \quad 13.197 \quad 10.541 \\
& \begin{array}{lllll}
6,9 & 1.986 & 12.387 & 10.980
\end{array} \\
& \begin{array}{llll}
6,8 & 1.426 & 11.588 & 11.429
\end{array} \\
& 6,7 \quad 0.773 \quad 10.809 \quad 11.888 \\
& \begin{array}{llll}
6,6 & 0.025 & 10.061 & 12.357
\end{array} \\
& 6.5 \quad-0.817 \quad 9.354 \quad 12.836 \\
& 6,4 \quad-1.753 \quad 8.699 \quad 13.326
\end{aligned}
$$




\begin{tabular}{|c|c|c|c|}
\hline 6,2 & -3.898 & 7.584 & 14.335 \\
\hline 6,1 & -5.099 & 7.147 & 14.855 \\
\hline 5,25 & 3.957 & 16.982 & 8.918 \\
\hline 5,14 & 3.957 & 16.394 & 9.256 \\
\hline $5,1.3$ & 3.884 & 15.560 & 9.721 \\
\hline 5,12 & 3.733 & 14.708 & 10.191 \\
\hline 5,11 & 3.470 & 13.831 & 10.674 \\
\hline 5,10 & 3.172 & 12.935 & 11.170 \\
\hline 5,9 & 2.750 & 12.030 & 11.680 \\
\hline 5,8 & 2.228 & 11.125 & 12.202 \\
\hline 5,7 & 2.601 & 10.230 & 12.738 \\
\hline 5,6 & 0.867 & 9.355 & 13.287 \\
\hline 5,5 & 0.024 & 8.512 & 13.849 \\
\hline 5,4 & -0.927 & 7.714 & 14.426 \\
\hline 3,3 & -1.987 & 6.971 & 15.015 \\
\hline 5,2 & $-3.1,5$ & 6.297 & 15.619 \\
\hline 5,1 & -4.426 & 5.704 & 16.237 \\
\hline 4,15 & 4.385 & 17.038 & 9.248 \\
\hline 4,14 & .4 .437 & 16.436 & 9.623 \\
\hline 4,13 & 4.437 & 15.624 & 10.107 \\
\hline 4,12 & $4.35,3$ & 14.654 & 10.675 \\
\hline 4,11 & 4.186 & 13.709 & 11.224 \\
\hline 4,10 & 3.925 & 12.734 & 11.790 \\
\hline 4,9 & 3.561 & 11.735 & 12.373 \\
\hline 4,8 & 3.089 & 10.723 & 12.973 \\
\hline 4,7 & $2.5: 3$ & 9.709 & 13.590 \\
\hline 4,6 & 1.799 & 8.702 & 14.224 \\
\hline $4 ; 5$ & 0.972 & 7.717 & 14.876 \\
\hline 4,4 & 0.021 & 6.765 & 15.545 \\
\hline $4: 3$ & $-1.056 \%$ & 5.861 & 16.232 \\
\hline 4,2 & -2.260 & 5.018 & 16.938 \\
\hline
\end{tabular}




\begin{tabular}{|c|c|c|c|}
\hline 4,1 & -3.588 & 4.252 & 17.661 \\
\hline 3,15 & 4.806 & 17.132 & 9.562 \\
\hline 3,14 & 4.914 & 16.520 & 9.973 \\
\hline 3,13 & 4.988 & 15.672 & 10.515 \\
\hline 3,12 & 4.983 & 14.698 & 11.120 \\
\hline 3,11 & 4.896 & 13.647 & 11.764 \\
\hline 3,10 & 4.711 & 12.595 & 12.402 \\
\hline 3,9 & 4.419 & 11.505 & 13.062 \\
\hline 3,8 & 4.012 & 10.387 & 13.743 \\
\hline 3,7 & 3.483 & 9.252 & 14.446 \\
\hline 3,6 & 2.824 & 8.111 & 15.171 \\
\hline 3,5 & 2.030 & 6.977 & 15.918 \\
\hline 3,4 & 1.096 & 5.863 & 16.687 \\
\hline 3,3 & 0.018 & 4.786 & 17.479 \\
\hline 3,2 & -1.204 & 3.760 & 18.294 \\
\hline 3,1 & -2.573 & 2.801 & 19.132 \\
\hline 2,15 & 5.218 & 17.262 & 9.859 \\
\hline 2,14 & 5.383 & 16.646 & 10.305 \\
\hline 2,13 & 5.538 & 15.769 & 10.907 \\
\hline 2,12 & 5.627 & 14.754 & 11.579 \\
\hline 2,11 & 5.627 & 13.780 & 12.205 \\
\hline 2,10 & 5.517 & 12.524 & 12.999 \\
\hline 2,9 & 5.309 & 11.348 & 13.739 \\
\hline 2,8 & 4.982 & 10.127 & 14.505 \\
\hline 2,7 & 4.525 & 8.872 & 15.299 \\
\hline 2,6 & 3.930 & 7.595 & 16.119 \\
\hline 2,5 & $3.1 \mathrm{d7}$ & 6.308 & 16.967 \\
\hline 2,4 & 2.290 & 5.027 & 17.842 \\
\hline 2,3 & 1.232 & 3.767 & 18.746 \\
\hline 2.2 & 0.010 & 2.545 & 19.679 \\
\hline 2,1 & -1.379 & 1.379 & 20.64 \\
\hline
\end{tabular}




$\begin{array}{llll}1,1.4 & 5.341 & 16.312 & 10.620 \\ 1,13 & 6.081 & 15.915 & 11.282 \\ 1,12 & 6.266 & 14.867 & 12.022 \\ 1,11 & 6.356 & 13.844 & 12.717 \\ 1,10 & 6.376 & 12.708 & 13.467 \\ 1,9 & 6.230 & 11.268 & 14.404 \\ 1,8 & 5.937 & 9.948 & 15.259 \\ 1,7 & 5.629 & 8.577 & 16.147 \\ 1,6 & 5.115 & 7.164 & 17.068 \\ 1,5 & 4.443 & 5.723 & 18.022 \\ 1,4 & 3.604 & 4.269 & 19.011 \\ 1,3 & 2.588 & 2.818 & 20.034 \\ 1,2 & 1.388 & 1.389 & 21.092 \\ 1,1 & 0.000 & 0.000 & 22.187\end{array}$


6.2 .5 . mirísicos

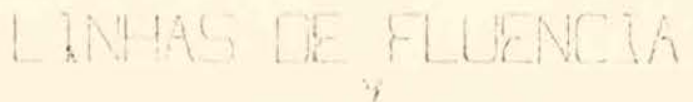
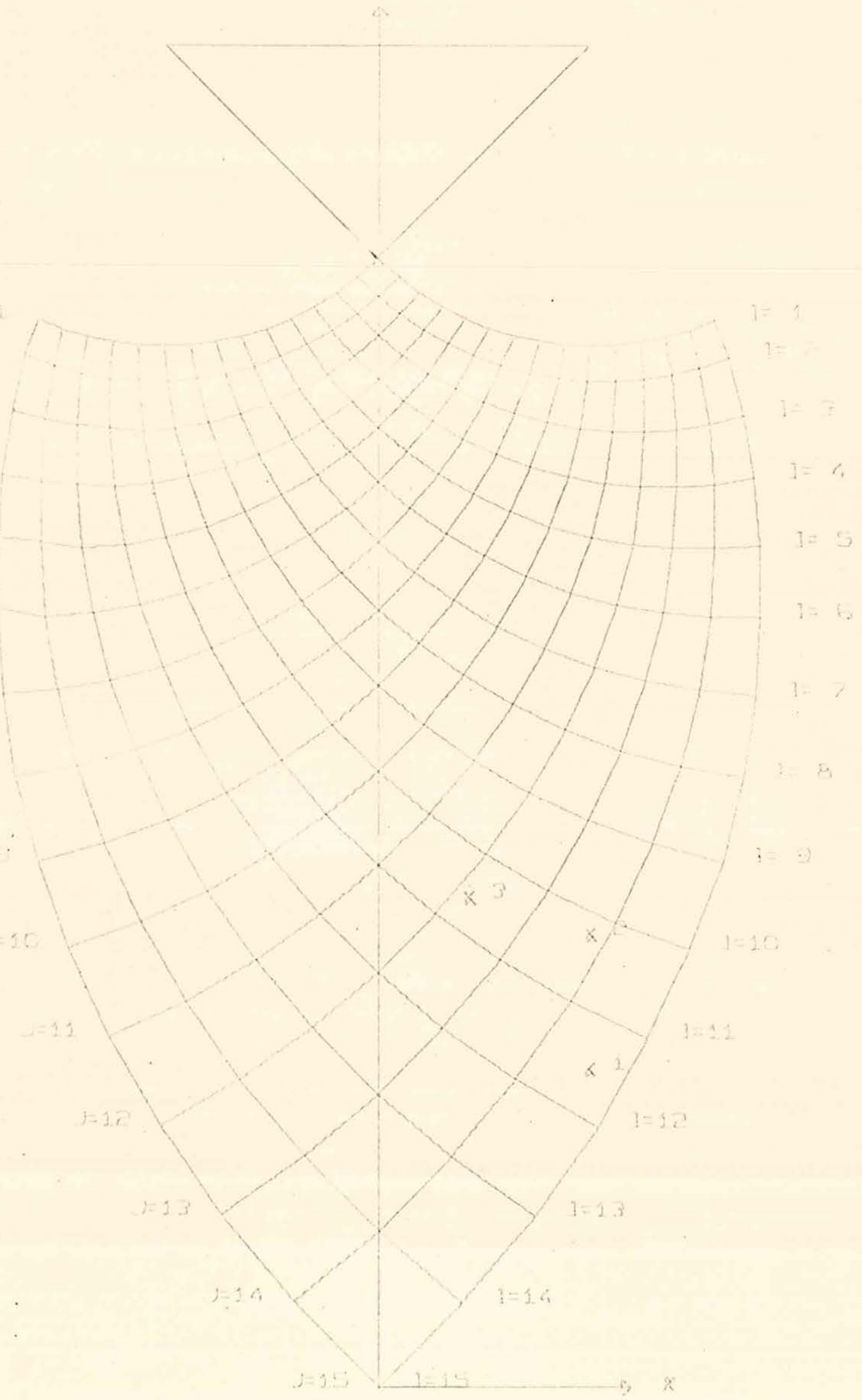


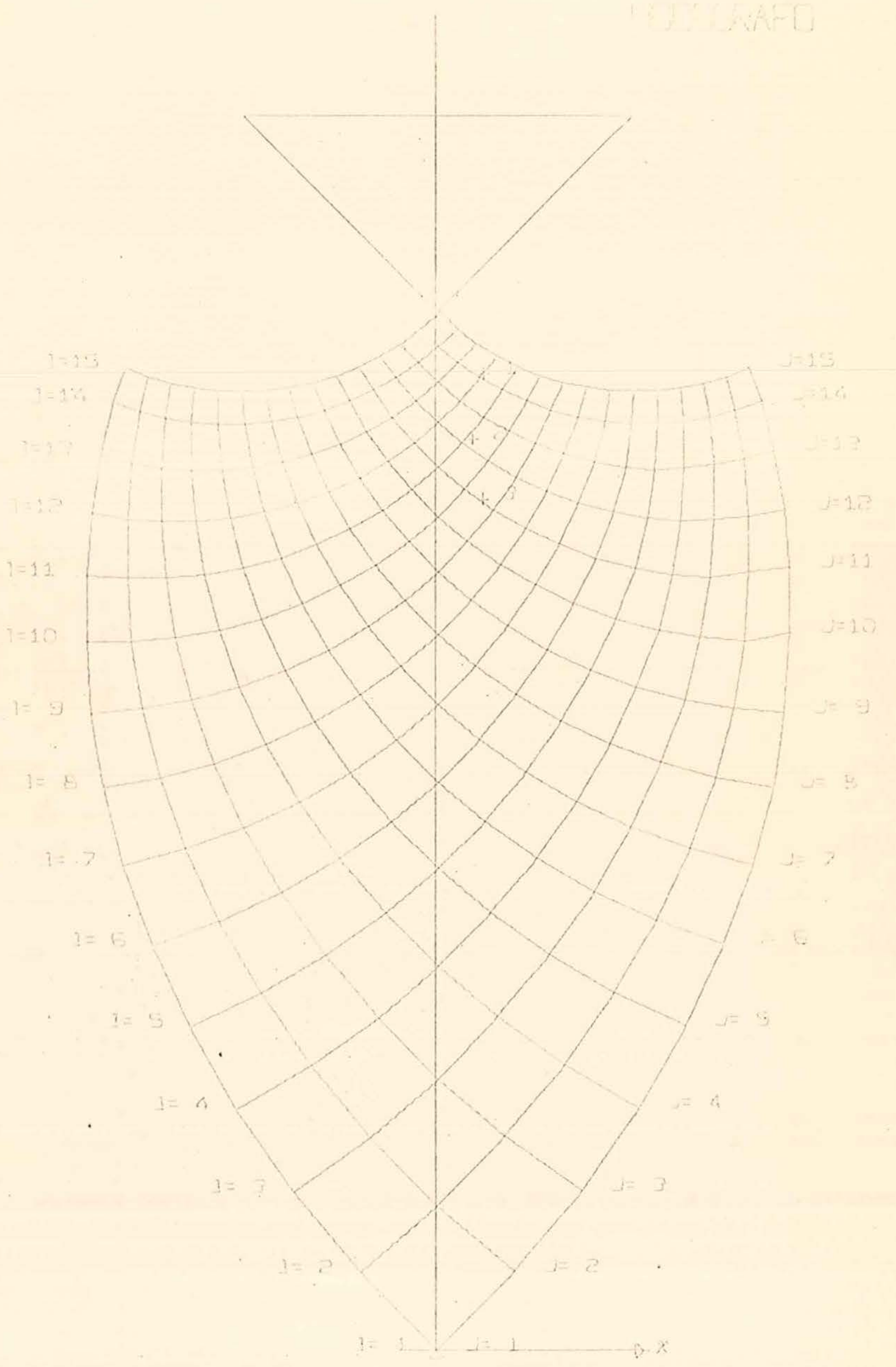


6.3- Modo de operar o programe

Para alimentar com dados os programas, cievemos proceder do seguinte modo:

a primejo cartĩo de dados será do tipo (2) com as se guinbes variávais.

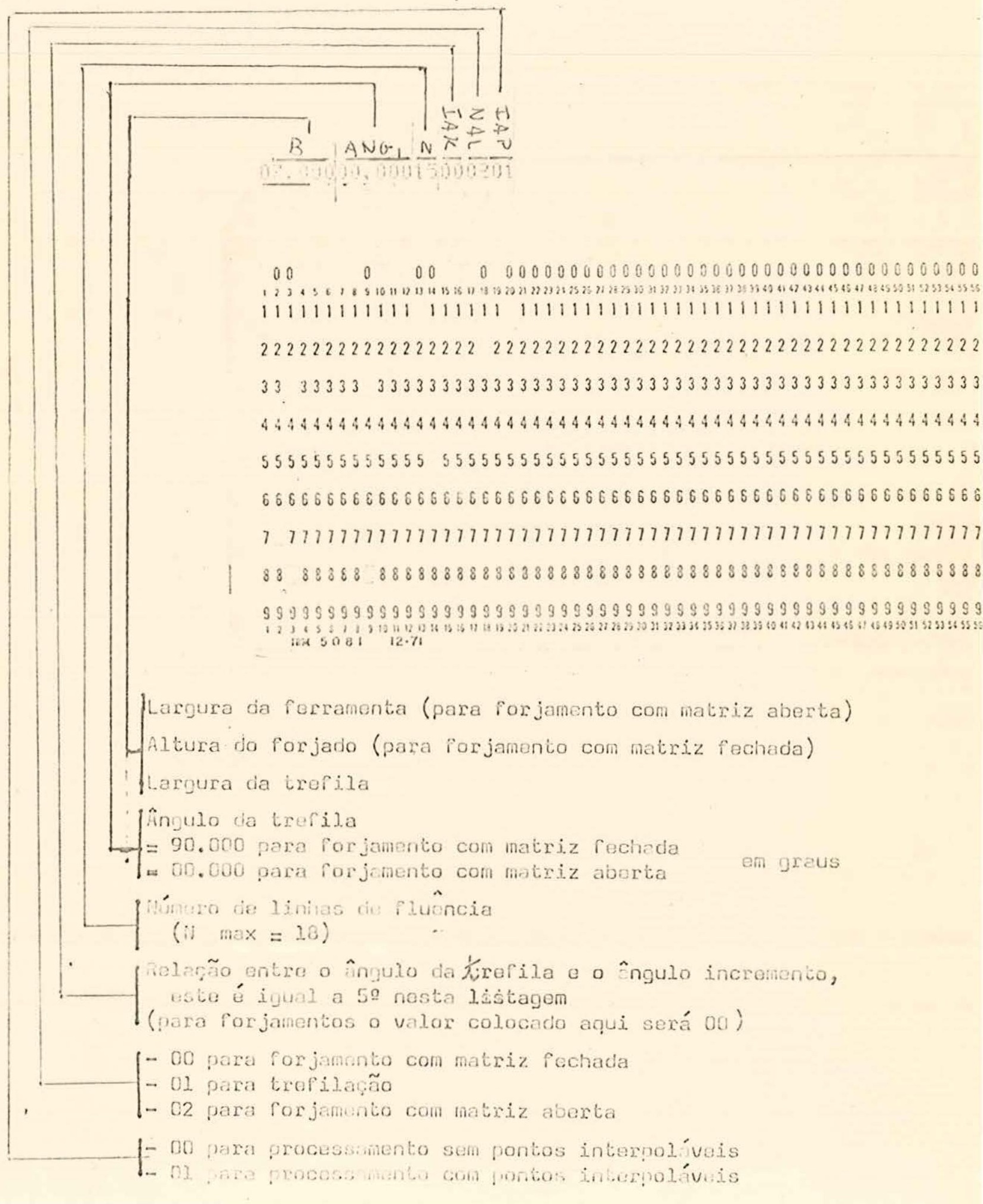


Se $T A P=1$, o cartão seguinte será preenchido assim:

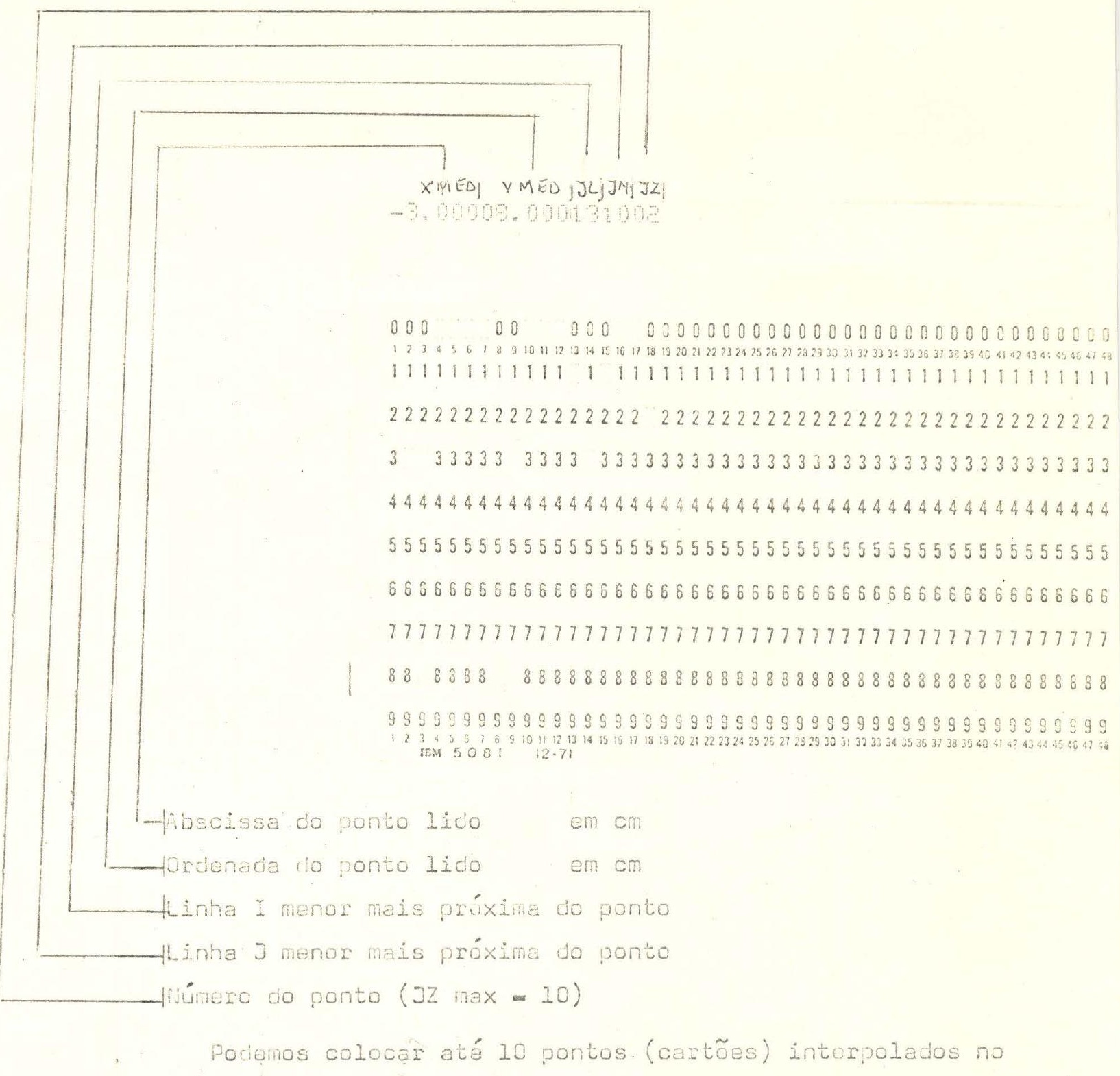

mesmo programa:

o último cartão tipo 2 necessariamente térá XMEo as J = $J i=J Z=0$.

Se quisermos outros valores para novos valores de $B$, Aing, etc, basta colocarmos cartões tipo l a sempre que nestes cartões colocarmos: IAP $=1$, colocarmos os pontos intorpoláveis (cartão tipo 2) 1000 após.

o último cartão do tipo l será necessariamente com to dos os valores iguais a zero. 
7 - Bibliografia

Foro, Hugh - Advanced mechanies of materials. London, Longmans, 1963. 6720 .

Wworw, Bent - A thoory of Blasticity for idnal frictionless materials. Copenhagen, Toknisk Forlag, 1965. 471p.

HEYman, 3., LECKIE, F. A., d. - Enqingering plasticity. Cambridge, University press, 1960. $706 p$.

HILL, R. - The mathematical theory of Dlasticity. Oxiord, Clarendion Press, 1971. 355p. (The Oxford Engineering Science series).

KoJAYASHI, S.; THOnSEH, E.G. - Approximate solutions to a problem of press forging. Journal of Engineering for Industry (Trans. of the ASnE - series 3). Now York, 81(3): 217-227, aug. 1959.

MENDELSON, Alexander - Plasticity: theory and application. Now York, Macmilian, 1968. 353p. (Macmilian series in Apllied Mechanics). NÁLI, A.; WAHL, A.M. - Plasticity: a mechanics of the plastic state of matter. New York, McGrat-Hili, 1931. 349p. (Engineering Societies Monographs).

PRAGER, William - A geometrical discussion of the slip line field in plane plastic flow. Transactions of the Royal Institute of Technolooy. Stockholn, Sweden, (65): 3-25, 1953.

PRAGER, Uilliam - Frobleme der plastizitytstheorie. Basel, Birkhduser, 1955. 100p.

PRAGER, William - Problèmes de plasticité thèorique; traduit par J. Kigffer et R. Epain. Paris, Dunod, 1958. 121p.

PRhGER, William ; HODGE, Jx., P.G. - Theorie ideal plastischer körper. Uien, Springer, 1954. 274p.

REHDER, otto Alfredo - Conformarão plástica: estudo macroscópico das deformacões permanentes nos materiais sólidos e sua aplicacão na technolonia mecânica. São Carlos, EESC, 1970。2v. 


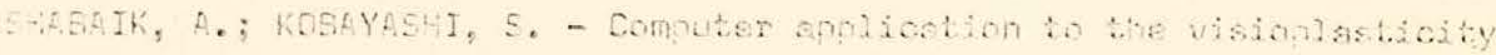

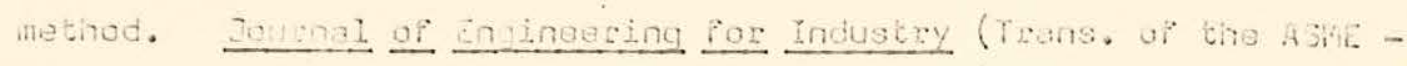
séries - B) Now Yoxk, 89 (2): 339-346, may, 1967.

Whensta, E.G. - New method for the construction of henckyprandt nets. Dournal of Anclied Mechanics (Trans. of the ASriE). New Yoxk, 24 (1): 81-34, mar. 1957 .

WESNER, JR., J. W. THeinstein, A.S. - Computerized relaxation applied to the plane-strain indenter. Journal of Basje Engineering (Trens. of the ASME - series D). New York, 91 (4): 816-821, dec. 1969. 